Supporting Information

\title{
The Synthesis and Preliminary Biological Evaluation of a Novel Steroid with Neurotrophic Activity, NGA0187
}

\author{
Zihao Hua, ${ }^{+}$David A. Carcache, ${ }^{+}$Yuan Tian, ${ }^{\S}$ Yue-Ming Li,${ }^{\S}$ and Samuel J. Danishefsky ${ }^{*+}$ \\ Contribution from ${ }^{+}$the Laboratory for Bioorganic Chemistry and ${ }^{\xi}$ Laboratory of Biochemistry and \\ Pharmacology, Sloan-Kettering Institute for Cancer Research, 1275 York Avenue, New York, New \\ York 10021; and ${ }^{\ddagger}$ Department of Chemistry, Columbia University, Havemeyer Hall, 3000 Broadway, \\ New York, New York 10027
}

Experimental Procedures

NMR Spectra

S13-51 
Analytical Equipment: ${ }^{1} \mathrm{H}$ and ${ }^{13} \mathrm{C}$ NMR spectra were recorded on a $400 \mathrm{MHz}$ or a500 $\mathrm{MHz}$ spectrometer in $\mathrm{CDCl}_{3}$ [referenced to $7.26 \mathrm{ppm}(\delta)$ for ${ }^{1} \mathrm{H}$ NMR and $77.0 \mathrm{ppm}$ for ${ }^{13} \mathrm{C} \mathrm{NMR}$ ]. Lowresolution mass spectra (ionspray, a variation of electrospray) were measured on API 100 spectrometer. High-resolution mass spectra (ESI) were acquired at the Mass Spectrometry Laboratory of University of Illinois Urbana-Champaign, Urbana, IL. Infrared spectra were obtained on 1600 FTIR spectrophotometer.

Compound 16: To a solution of $\mathbf{1 1}(2.5 \mathrm{mg}, 0.0041 \mathrm{mmol})$ in $\mathrm{CH}_{2} \mathrm{Cl}_{2}(0.5 \mathrm{~mL})$ at room temperature, was added Dess-Martin periodinane $(2.6 \mathrm{mg}, 0.061 \mathrm{mmol})$. The resultant mixture was stirred at room temperature for $3 \mathrm{~h}$ before being quenched with saturated $\mathrm{NaHCO}_{3}-\mathrm{Na}_{2} \mathrm{SO}_{3}(1: 1,3.0 \mathrm{~mL})$. The mixture was extracted with EtOAc $(3.0 \mathrm{~mL}$ x 5). The combined extracts were washed with brine, dried over $\mathrm{MgSO}_{4}$ and concentrated in vacuo to afford crude compound 16 (1.9 $\left.\mathrm{mg}\right)$ as light yellow solid. ${ }^{1} \mathrm{H}$ NMR (400 MHz, $\left.\mathrm{CDCl}_{3}\right) \delta 0.05$ (s, 6H), 0.07 (s, 3H), 0.15 (s, 3H), 0.88 (s, 9H), 0.90 (s, 3H), $1.11(\mathrm{~s}, 3 \mathrm{H}), 1.25-2.18(\mathrm{~m}, 15 \mathrm{H}), 3.50-3.60(\mathrm{~m}, 1 \mathrm{H}), 3.72-3.98(\mathrm{~m}, 6 \mathrm{H}), 4.46(\mathrm{br}, 1 \mathrm{H}) ; \quad$ LRMS m/z $631.3\left[(\mathrm{M}+\mathrm{Na})^{+}\right.$; calcd for $\left.\mathrm{C}_{33} \mathrm{H}_{60} \mathrm{O}_{6} \mathrm{NaSi}_{2}: 631.4\right]$.

Compound 17: To a solution of compound $11(73 \mathrm{mg}, 0.12 \mathrm{mmol})$, triethylamine $(0.09 \mathrm{~mL}, 0.60$ mmol) and DMAP $(1 \mathrm{mg}, 0.012 \mathrm{mmol})$ in $\mathrm{CH}_{2} \mathrm{Cl}_{2}(1.2 \mathrm{~mL})$ at $0{ }^{\circ} \mathrm{C}$, was added benzol chloride $(0.04$ $\mathrm{mL}, 0.36 \mathrm{mmol}$ ). The mixture was allowed to warm to room temperature with stirring and diluted with $\mathrm{Et}_{2} \mathrm{O}$ after $4.5 \mathrm{~h}$. The solution was washed with saturated $\mathrm{NaHCO}_{3}$ and brine, dried over anhydrous $\mathrm{MgSO}_{4}$. The solvent was removed under reduced pressure and the crude was subjected to column chromatography on silica gel (15:1 to 10:1 hexanes-EtOAc) to afford $\mathbf{1 7}$ as off-white solid (42 mg, 49\%). ${ }^{1} \mathrm{H}$ NMR (400 MHz, $\mathrm{CDCl}_{3}$ ) $\delta-0.05$ (s, 3H), -0.02 (s, 3H), 0.09 (s, 3H), 0.14 (s, 3H), $0.80(\mathrm{~s}, 9 \mathrm{H}), 0.91$ (s, 3H), $1.12(\mathrm{~s}, 3 \mathrm{H}), 1.15(\mathrm{~s}, 3 \mathrm{H}), 0.90-2.35(\mathrm{~m}, 16 \mathrm{H}), 3.42-3.50(\mathrm{~m}, 2 \mathrm{H}), 3.81-$ $3.92(\mathrm{~m}, 4 \mathrm{H}), 4.40(\mathrm{br}, 1 \mathrm{H}), 4.98(\mathrm{dd}, \mathrm{J}=10.5,9.1 \mathrm{~Hz}, 1 \mathrm{H}), 7.43$ (t, J = $7.7 \mathrm{~Hz}, 2 \mathrm{H}), 7.56$ (t, J = 7.4 $\mathrm{Hz}, 1 \mathrm{H}), 8.04(\mathrm{~d}, \mathrm{~J}=7.4 \mathrm{~Hz}, 1 \mathrm{H}) ; \mathrm{LRMS} \mathrm{m} / \mathrm{z} 737.5\left[(\mathrm{M}+\mathrm{Na})^{+}\right.$; calcd for $\left.\mathrm{C}_{40} \mathrm{H}_{66} \mathrm{O}_{7} \mathrm{NaSi}_{2}: 738.1\right]$.

Compound 18: A mixture of compound 17 (2.5 mg, 0.0035 mmol), Dess-Martin Periodinane (4.4 mg, $0.010 \mathrm{mmol})$ in $\mathrm{CH}_{2} \mathrm{Cl}_{2}(0.5 \mathrm{~mL})$ was stirred at room temperature for $1 \mathrm{~h}$, a $1: 1$ mixture of $\mathrm{NaHCO}_{3}-$ $\mathrm{Na}_{2} \mathrm{~S}_{2} \mathrm{O}_{3}(3 \mathrm{~mL})$ was added and stirred for $10 \mathrm{~min}$. The mixture was extracted with EtOAc (5 mL x 3), washed with brine and dried over anhydrous $\mathrm{MgSO}_{4}$. The solvent was removed in vacuo to afford 18 
as crude solid $(3.3 \mathrm{mg})$, which was used for next step without further purification. ${ }^{1} \mathrm{H}$ NMR (400 $\left.\mathrm{MHz}, \mathrm{CDCl}_{3}\right) \delta 0.03(\mathrm{~s}, 3 \mathrm{H}), 0.05$ (s, 3H), 0.12 (s, 3H), 0.17 (s, 3H), 0.86 (s, 9H), 0.94 (s, 9H), 1.07 (s, 3H), 1.37 (s, 3H), 0.80-2.35 (m, 16H), 2.89 (dd, J = 11.5, $11.3 \mathrm{~Hz}, 1 \mathrm{H}), 3.54-3.59(\mathrm{~m}, 1 \mathrm{H}), 3.80-$ $3.91(\mathrm{~m}, 4 \mathrm{H}), 4.43$ (br, 1H), 5.19 (d, J = 12.7 Hz, 1H), 7.44 (t, J = $7.7 \mathrm{~Hz}, 2 \mathrm{H}), 7.56$ (t, J = 7.2 Hz, $1 \mathrm{H}), 8.06(\mathrm{~d}, \mathrm{~J}=7.2 \mathrm{~Hz}, 2 \mathrm{H})$.

Compound 19: A mixture of crude compound $18(2.0 \mathrm{mg})$ in $1 \% \mathrm{KOH}-\mathrm{MeOH}(0.6 \mathrm{~mL})$ was stirred at room temperature for $2.5 \mathrm{~h}$. The solvent was removed under reduced pressure and then diluted with water $(5 \mathrm{~mL})$. The mixture was extracted with EtOAc $(5 \mathrm{~mL}$ x 3$)$, washed with brine and dried over anhydrous $\mathrm{MgSO}_{4}$. The solvent was removed in vacuo to afford 19 as crude solid (1. mg2), which was subjected to ${ }^{1} \mathrm{H}$ NMR and mass analysis without further purification. ${ }^{1} \mathrm{H}$ NMR (400 $\mathrm{MHz}, \mathrm{CDCl}_{3}$ ) $\delta 0.05(\mathrm{~s}, 3 \mathrm{H}), 0.07(\mathrm{~s}, 3 \mathrm{H}), 0.10(\mathrm{~s}, 3 \mathrm{H}), 0.15(\mathrm{~s}, 3 \mathrm{H}), 0.70-2.35(\mathrm{~m}, 41 \mathrm{H}), 2.77-2.80(\mathrm{~m}, 1 \mathrm{H}), 3.51-$ $3.67(\mathrm{~m}, 2 \mathrm{H}), 3.82-3.93(\mathrm{~m}, 4 \mathrm{H}), 4.69(\mathrm{br}, 1 \mathrm{H})$; LRMS m/z $631.3\left[(\mathrm{M}+\mathrm{Na})^{+}\right.$; calcd for $\mathrm{C}_{33} \mathrm{H}_{60} \mathrm{O}_{6} \mathrm{NaSi}_{2}$ : 631.4].

Compound 29: To a suspension of $\mathrm{CrCl}_{2}(5.0 \mathrm{~g}, 40.68 \mathrm{mmol})$ in $\mathrm{THF}(50 \mathrm{~mL})$ at $0{ }^{\circ} \mathrm{C}$, was added a solution of $\mathrm{CHI}_{3}(5.4 \mathrm{~g}, 13.71 \mathrm{mmol})$ and $(R)$-2,3-dimethylbutanal (28) ${ }^{1}(681 \mathrm{mg}, 6.80 \mathrm{mmol})$ dropwiase. The mixture was stirred at $0{ }^{\circ} \mathrm{C}$ for $3 \mathrm{~h}$ and room temperature for $1 \mathrm{~h}$. The reaction was quenched with water and extracted with water. The extract was concentrated carefully and subjected to chromatography on silica gel (pentane as eluant). The fractions with desired vinyl iodide $\mathbf{2 9}$ was collected and concentrated carefully to give a pentane solution $(\sim 690 \mathrm{mg} 29$ included, $45 \%$ yield, $\mathrm{E} / \mathrm{Z}=93: 7) .{ }^{1} \mathrm{H}$ NMR $\left(400 \mathrm{MHz}, \mathrm{CDCl}_{3}\right) \delta 0.85(\mathrm{~d}, \mathrm{~J}=5.1 \mathrm{~Hz}, 3 \mathrm{H}), 0.87(\mathrm{~d}, \mathrm{~J}=5.1 \mathrm{~Hz}, 3 \mathrm{H}), 0.97(\mathrm{~d}$, $\mathrm{J}=6.9 \mathrm{~Hz}, 3 \mathrm{H}), 1.50-1.62(\mathrm{~m}, 1 \mathrm{H}), 1.97-2.05(\mathrm{~m}, 1 \mathrm{H}), 5.93(\mathrm{~d}, \mathrm{~J}=14.4 \mathrm{~Hz}, 1 \mathrm{H}), 6.43(\mathrm{dd}, \mathrm{J}=14.4$, $8.5 \mathrm{~Hz}, 1 \mathrm{H}) ;{ }^{13} \mathrm{C} \mathrm{NMR}\left(100 \mathrm{MHz}, \mathrm{CDCl}_{3}\right) \delta 16.5,19.5,19.8,32.5,47.0,73.6,150.9$.

Compound 36: To a stirred solution of sterol $7^{2}(1.49 \mathrm{~g}, 4.30 \mathrm{mmol})$ in $\mathrm{CH}_{2} \mathrm{Cl}_{2}(30 \mathrm{~mL})$ at $0{ }^{\circ} \mathrm{C}$, was added pyridine $(2.1 \mathrm{~mL}, 25.81 \mathrm{mmol})$ and TESOTf $(2.9 \mathrm{~mL}, 12.91 \mathrm{mmol})$. The mixture was stirred at $0{ }^{\circ} \mathrm{C}$ for $1 \mathrm{~h}$ before it was quenched by addition of water $(30 \mathrm{~mL})$. The organic layer was separated

\footnotetext{
${ }^{1}$ Tsuda, K.; Kishida, Y.; Hayazu, R. J. Am. Chem. Soc. 1960, 82, 3396-3399.

2 (a) Mase, T.; Ichita, J.; Marino, J. P.; Koreeda, M. Tetrahedron Lett. 1989, 30, 2075-2078; (b) Moon, S. S.; Stuhmiller, L. M.; McMorris, T. C. J. Org. Chem. 1989, 54, 26-28; (c) Moon, S.; Stuhmiller, L. M.; Chadha, R. K.; McMorris, T. C. Tetrahedron 1990, 46, 2287-2306.
} 
and the aqueous layer was extracted with $\mathrm{Et}_{2} \mathrm{O}(50 \mathrm{~mL} \times 3)$. The combined organic layers were washed with brine and dried over anhydrous $\mathrm{MgSO}_{4}$. The solution was concentrated under reduced pressure to give a crude oil, which was purified with flash column chromatography on silica gel (20:1 hexanes-EtOAc) to afford $36(2.13 \mathrm{~g}, 87 \%)$ as white solid. $[\alpha]_{\mathrm{D}}^{24}-36.7\left(c 0.24, \mathrm{CHCl}_{3}\right) ;{ }^{1} \mathrm{H} \mathrm{NMR}(400$ $\left.\mathrm{MHz}, \mathrm{CDCl}_{3}\right) \delta$ 0.53-0.72 (m, $\left.12 \mathrm{H}\right), 0.96(\mathrm{t}, \mathrm{J}=7.9 \mathrm{~Hz}, 9 \mathrm{H}), 0.97(\mathrm{t}, \mathrm{J}=8.0 \mathrm{~Hz}, 9 \mathrm{H}), 1.06(\mathrm{~s}, 3 \mathrm{H})$, 1.19 (s, 3H), 0.90-2.35 (m, 17H), 3.44-3.49 (m, 1H), 3.80-3.92 (m, 4H), 4.54 (d, J = 3.1 Hz, 1H), 5.16 $(\mathrm{t}, \mathrm{J}=2.0 \mathrm{~Hz}, 1 \mathrm{H}) ;{ }^{13} \mathrm{C} \mathrm{NMR}\left(100 \mathrm{MHz}, \mathrm{CDCl}_{3}\right) \delta 5.3,5.9,7.3,7.6,16.6,22.1,23.2,29.3,32.3,34.4$, $36.7,37.1,39.5,42.4,45.4,53.2,54.8,64.8,65.5,69.5,72.1,120.0,120.0,143.2$; FTIR (neat) 2951, 2875, 1456, 1237, 1166, 1101, 1048, 1011, 972, 799, $727 \mathrm{~cm}^{-1}$; HRMS (ESI) m/z 577.4108 [(M+H) calcd for $\mathrm{C}_{33} \mathrm{H}_{61} \mathrm{O}_{4} \mathrm{Si}_{2}:$ : 577.4108].

Compound 8: ${ }^{1} \mathrm{H}$ NMR (400 MHz, $\left.\mathrm{CDCl}_{3}\right) \delta 0.01$ (s, 6H), 0.06 (s, 3H), 0.13 (s, 3H), 0.89 (s, 9H), $0.90(\mathrm{~s}, 9 \mathrm{H}), 1.07(\mathrm{~s}, 3 \mathrm{H}), 1.21(\mathrm{~s}, 3 \mathrm{H}), 0.90-2.35(\mathrm{~m}, 17 \mathrm{H}), 3.45-3.50(\mathrm{~m}, 1 \mathrm{H}), 3.82-3.91(\mathrm{~m}, 4 \mathrm{H})$, 4.44 (br, 1H), 5.16 (br, 1H); LRMS m/z 599.5 [(M+Na) ${ }^{+}$; calcd for $\left.\mathrm{C}_{33} \mathrm{H}_{60} \mathrm{O}_{4} \mathrm{NaSi}_{2}: 600.0\right]$.

Compound 37: A suspension of finely grounded $\mathrm{CrO}_{3}(7.93 \mathrm{~g}, 79.32 \mathrm{mmol})$ in $\mathrm{CH}_{2} \mathrm{Cl}_{2}(70 \mathrm{~mL})$ was cooled to $-25{ }^{\circ} \mathrm{C}$, to this solution was added 3,5-dimethylpyrazole $(7.63 \mathrm{~g}, 79.32 \mathrm{mmol})$ in one portion. The mixture was stirred at this temperature for 20 minutes to give a dark-red solution. To this mixture, compound 36 (4.57 g, $7.93 \mathrm{mmol})$ in $\mathrm{CH}_{2} \mathrm{Cl}_{2}(50 \mathrm{~mL})$ was added, the reaction mixture was stirred at $-25{ }^{\circ} \mathrm{C}$ for $4 \mathrm{~h}$ and then diluted with $\mathrm{Et}_{2} \mathrm{O}(500 \mathrm{~mL})$. The solid was filtered off through a short pad of celite. The filtrate was washed with water, dried over anhydrous $\mathrm{MgSO}_{4}$ and concentrated in vacuo. The residue was flash chromatographed with silica gel (15:1 hexanes-EtOAc) to afford $37(2.39 \mathrm{~g}, 51 \%)$ as a white solid. $[\alpha]_{\mathrm{D}}^{24}-60.0\left(c 0.31, \mathrm{CHCl}_{3}\right) ;{ }^{1} \mathrm{H}$ NMR $\left(400 \mathrm{MHz}, \mathrm{CDCl}_{3}\right)$ $\delta$ 0.57-0.72 (m, 12H), $0.96(\mathrm{t}, \mathrm{J}=7.8 \mathrm{~Hz}, 9 \mathrm{H}), 0.98(\mathrm{t}, \mathrm{J}=7.8 \mathrm{~Hz}, 9 \mathrm{H}), 1.10$ (s, 3H), 1.28 (s, 3H), 1.20-1.35 (m, 1H), 1.49-2.25 (m, 12H), 2.40-2.47 (m, 2H), $2.58(\mathrm{t}, \mathrm{J}=11.6 \mathrm{~Hz}, 1 \mathrm{H}), 3.58-3.61(\mathrm{~m}$, 1H), 3.79-3.92 (m, 4H), $4.45(\mathrm{~d}, \mathrm{~J}=2.9 \mathrm{~Hz}, 1 \mathrm{H}), 5.62(\mathrm{~d}, \mathrm{~J}=1.6 \mathrm{~Hz}, 1 \mathrm{H}) ;{ }^{13} \mathrm{C} \mathrm{NMR}(100 \mathrm{MHz}$, $\left.\mathrm{CDCl}_{3}\right) \delta 5.3,5.9,7.2,7.5,17.1,20.8,25.4,31.8,34.2,35.5,38.5,38.5,42.1,42.7,46.6,47.6,55.4$, 64.7, 65.6, 69.1 , 70.6, 119.1, 126.0, 169.2, 201.9; FTIR (neat) 2952, 2876, 1664, 1457, 1100, 1053, 1009, 965, $739 \mathrm{~cm}^{-1}$; HRMS (ESI) m/z 591.3892 [(M+H) ${ }^{+}$; calcd for $\left.\mathrm{C}_{33} \mathrm{H}_{59} \mathrm{O}_{5} \mathrm{Si}_{2}: 591.3901\right]$. Compound 9: ${ }^{1} \mathrm{H}$ NMR (400 MHz, $\left.\mathrm{CDCl}_{3}\right) \delta 0.06(\mathrm{~s}, 6 \mathrm{H}), 0.10(\mathrm{~s}, 3 \mathrm{H}), 0.15$ (s, 3H), 0.88 (s, 9H), $0.91(\mathrm{~s}, 9 \mathrm{H}), 1.12(\mathrm{~s}, 3 \mathrm{H}), 1.29(\mathrm{~s}, 3 \mathrm{H}), 1.63-2.33(\mathrm{~m}, 15 \mathrm{H}), 3.46-3.53(\mathrm{~m}, 1 \mathrm{H}), 3.81-3.93(\mathrm{~m}, 4 \mathrm{H})$, 4.42 (br, 1H), 5.17 (br, 1H); LRMS m/z 591.4 [(M+H) ${ }^{+}$; calcd for $\left.\mathrm{C}_{33} \mathrm{H}_{59} \mathrm{O}_{5} \mathrm{Si}_{2}: 592.0\right]$. 
Compound 38: To a cooled solution of $37(1.97 \mathrm{~g}, 3.33 \mathrm{mmol})$ and $\mathrm{CeCl}_{3} .7 \mathrm{H}_{2} \mathrm{O}(2.48 \mathrm{~g}, 6.67 \mathrm{mmol})$ in THF-MeOH $(5: 2,35 \mathrm{~mL})$ at $-70{ }^{\circ} \mathrm{C}$, was added $\mathrm{NaBH}_{4}(0.25 \mathrm{~g}, 6.67 \mathrm{mmol})$. The reaction was stirred at this temperature for 40 minutes and quenched with aqueous $\mathrm{NH}_{4} \mathrm{Cl}$. The mixture was extracted with $\mathrm{Et}_{2} \mathrm{O}(150 \mathrm{~mL} \times 3)$. The extract was washed with brine, dried over anhydrous $\mathrm{MgSO}_{4}$ and concentrated in vacuo to give $38(1.90 \mathrm{~g}, 96 \%)$ as white sticky solid, which is pure enough for next reaction without further purification. $[\alpha]_{\mathrm{D}}^{24}-3.8\left(\right.$ c $\left.0.16, \mathrm{CHCl}_{3}\right) ;{ }^{1} \mathrm{H} \mathrm{NMR}\left(400 \mathrm{MHz}, \mathrm{CDCl}_{3}\right)$ $\delta 0.60(\mathrm{q}, \mathrm{J}=8.0 \mathrm{~Hz}, 6 \mathrm{H}), 0.65(\mathrm{dq}, \mathrm{J}=7.6,2.9 \mathrm{~Hz}, 6 \mathrm{H}), 0.96(\mathrm{t}, \mathrm{J}=7.9 \mathrm{~Hz}, 9 \mathrm{H}), 0.97$ (t, J = 7.8 Hz, 9H), 1.09 (s, 3H), 1.25 (s, 3H), 1.04-1.16 (m, 2H), 1.48-2.39 (m, 13H), 3.46-3.51 (m, 1H), 3.81-3.93 $(\mathrm{m}, 5 \mathrm{H}), 4.43(\mathrm{~d}, \mathrm{~J}=3.0 \mathrm{~Hz}, 1 \mathrm{H}), 5.17(\mathrm{~s}, 1 \mathrm{H}) ;{ }^{13} \mathrm{C} \mathrm{NMR}\left(100 \mathrm{MHz}, \mathrm{CDCl}_{3}\right) \delta$ 5.3, 5.9, 7.2, 7.5, 16.6, 22.2, 24.8, 32.3, 34.4, 36.3, 37.1, 39.1, 42.0, 45.7, 53.3, 53.7, 64.8, 65.5, 69.3, 71.7, 74.3, 119.6, 123.8, 146.6; FTIR (neat) 2952, 2876, 1652, 1558, 1540, 1506, 1456, 1417, 1238, 1166, 1099, 1053, 1010, 968, 805, $727 \mathrm{~cm}^{-1}$; HRMS (ESI) $\mathrm{m} / \mathrm{z} 615.3896\left[(\mathrm{M}+\mathrm{Na})^{+}\right.$; calcd for $\mathrm{C}_{33} \mathrm{H}_{60} \mathrm{O}_{5} \mathrm{NaSi}_{2}$ : 615.3877].

Compound 10: ${ }^{1} \mathrm{H}$ NMR (400 MHz, $\left.\mathrm{CDCl}_{3}\right) \delta 0.04(\mathrm{~s}, 6 \mathrm{H}), 0.09(\mathrm{~s}, 3 \mathrm{H}), 0.14(\mathrm{~s}, 3 \mathrm{H}), 0.85(\mathrm{~s}, 9 \mathrm{H})$, $0.92(\mathrm{~s}, 9 \mathrm{H}), 1.11(\mathrm{~s}, 3 \mathrm{H}), 1.28(\mathrm{~s}, 3 \mathrm{H}), 1.60-2.39(\mathrm{~m}, 15 \mathrm{H}), 3.47-3.51(\mathrm{~m}, 1 \mathrm{H}), 3.82-3.93(\mathrm{~m}, 5 \mathrm{H})$, 4.42 (br, 1H), 5.17 (br, 1H); LRMS m/z 615.6 [(M+Na) ${ }^{+}$; calcd for $\left.\mathrm{C}_{33} \mathrm{H}_{60} \mathrm{O}_{5} \mathrm{NaSi}_{2}: 616.0\right]$.

Compound 39: To a solution of 38 in $\mathrm{CH}_{2} \mathrm{Cl}_{2}$-pyridine $(9: 1,1 \mathrm{~mL})$ at room temperature, were added acetic anhydride $(0.05 \mathrm{~mL}, 0.53 \mathrm{mmol})$ and DMAP $(1.6 \mathrm{mg}, 0.013 \mathrm{mmol})$. The mixture was stirred at room temperature for $4 \mathrm{~h}$. The solvent was evaporated in vacuo and the residue was subjected to a silica gel column (10:1 hexanes-EtOAc) to give $39(83 \mathrm{mg}, 98 \%)$ as white sticky solid. $[\alpha]_{\mathrm{D}}^{24}-15.8(c$ 0.24, $\left.\mathrm{CHCl}_{3}\right) ;{ }^{1} \mathrm{H} \mathrm{NMR}\left(400 \mathrm{MHz}, \mathrm{CDCl}_{3}\right) \delta 0.58(\mathrm{q}, \mathrm{J}=7.9 \mathrm{~Hz}, 6 \mathrm{H}), 0.65(\mathrm{dq}, \mathrm{J}=8.0 \mathrm{~Hz}, 3.2 \mathrm{~Hz}$, $6 \mathrm{H}), 0.95(\mathrm{t}, \mathrm{J}=7.9 \mathrm{~Hz}, 9 \mathrm{H}), 0.97(\mathrm{t}, \mathrm{J}=8.0 \mathrm{~Hz}, 9 \mathrm{H}), 1.08(\mathrm{~s}, 3 \mathrm{H}), 1.11-1.18(\mathrm{~m}, 2 \mathrm{H}), 1.26(\mathrm{~s}, 3 \mathrm{H})$, 1.48-2.36 (m, 13H), $2.02(\mathrm{~s}, 3 \mathrm{H}), 3.43-3.50(\mathrm{~m}, 1 \mathrm{H}), 3.80-3.90(\mathrm{~m}, 4 \mathrm{H}), 4.45(\mathrm{~d}, \mathrm{~J}=3.0 \mathrm{~Hz}, 1 \mathrm{H}), 5.08$ $(\mathrm{d}, \mathrm{J}=4.9 \mathrm{~Hz}, 1 \mathrm{H}), 5.14(\mathrm{~s}, 1 \mathrm{H}) ;{ }^{13} \mathrm{C} \mathrm{NMR}\left(100 \mathrm{MHz}, \mathrm{CDCl}_{3}\right) \delta$ 5.2, 5.9, 7.2, 7.6, 16.6, 22.0, 22.0, 23.8, 32.1, 34.3, 34.7, 36.3, 37.1, 38.9, 42.0, 45.7, 51.9, 53.5, 64.8, 65.6, 69.2, 71.5, 76.9, 119.4, 119.4, 148.4, 171.6; FTIR (neat) 2952, 2876, 1734, 1652, 1558, 1540, 1506, 1457, 1372, 1241, 1167, 1090, 1054, 1014, 968, $733 \mathrm{~cm}^{-1}$; HRMS (ESI) m/z $657.3971\left[(\mathrm{M}+\mathrm{Na})^{+}\right.$; calcd for $\mathrm{C}_{35} \mathrm{H}_{62} \mathrm{O}_{6} \mathrm{NaSi}_{2}$ : 657.3983]. 
Compound 40: To a solution of $39(1.84 \mathrm{~g}, 2.91 \mathrm{mmol})$ in THF $(20 \mathrm{~mL})$ at $0{ }^{\circ} \mathrm{C}$, was added $\mathrm{BH}_{3}(1.0$ $\mathrm{M}$ in THF, $8.72 \mathrm{~mL}$ ). The mixture was stirred and allowed to warm to room temperature overnight. $30 \% \mathrm{H}_{2} \mathrm{O}_{2}(2.2 \mathrm{~mL})$ and $10 \% \mathrm{NaOH}(2.2 \mathrm{~mL})$ was added and the mixture was stirred at room temperature for $2 \mathrm{~h}$. The reaction was quenched with saturated $\mathrm{NH}_{4} \mathrm{Cl}$ and extracted with $\mathrm{Et}_{2} \mathrm{O}$. The combined extract was washed with brine, dried over anhydrous $\mathrm{MgSO}_{4}$ and concentrated in vacuo. The crude oil was subjected to flash chromatography on silica gel (10:1, 5:1 then 2:1 hexanes-EtOAc) to give $40(1.35 \mathrm{~g}, 76 \%)$ as white solid. $[\alpha]_{\mathrm{D}}^{24}+40.9\left(\right.$ c $\left.0.23, \mathrm{CHCl}_{3}\right) ;{ }^{1} \mathrm{H} \mathrm{NMR}\left(400 \mathrm{MHz}, \mathrm{CDCl}_{3}\right)$ $\delta$ 0.55-0.66 (m, 12H), $0.75(\mathrm{dd}, \mathrm{J}=11.4,2.8 \mathrm{~Hz}, 1 \mathrm{H}), 0.95(\mathrm{t}, \mathrm{J}=7.8 \mathrm{~Hz}, 9 \mathrm{H}), 0.96(\mathrm{t}, \mathrm{J}=8.5 \mathrm{~Hz}$, 9H), 0.99 (s, 3H), $1.07(\mathrm{~s}, 3 \mathrm{H}), 1.28-1.99(\mathrm{~m}, 13 \mathrm{H}), 2.20(\mathrm{t}, \mathrm{J}=4.1 \mathrm{~Hz}, 2 \mathrm{H}), 3.12(\mathrm{dt}, \mathrm{J}=8.8,3.8 \mathrm{~Hz}$, $1 \mathrm{H}), 3.23-3.29(\mathrm{~m}, 1 \mathrm{H}), 3.45-3.52(\mathrm{~m}, 1 \mathrm{H}), 3.77-3.91(\mathrm{~m}, 4 \mathrm{H}), 4.38(\mathrm{~d}, \mathrm{~J}=2.7 \mathrm{~Hz}, 1 \mathrm{H}) ;{ }^{13} \mathrm{C} \mathrm{NMR}$ $\left(100 \mathrm{MHz}, \mathrm{CDCl}_{3}\right) \delta 5.3,5.9,7.3,7.5,16.5,17.2,25.7,31.5,32.6,34.4,36.1,37.4,38.2,38.7,46.3$, 48.8, 52.3, 56.9, 64.8, 65.5, 69.0, 71.7, 75.0, 81.9, 119.2; FTIR (neat) 3357, 2953, 2875, 1652, 1558, 1540, 1456, 1237, 1088, 1005, 836, $729 \mathrm{~cm}^{-1}$; HRMS (ESI) $\mathrm{m} / \mathrm{z} 611.4152\left[(\mathrm{M}+\mathrm{H})^{+}\right.$; calcd for $\left.\mathrm{C}_{33} \mathrm{H}_{63} \mathrm{O}_{6} \mathrm{Si}_{2}: 611.4163\right]$.

Compound 11: ${ }^{1} \mathrm{H}$ NMR (400 MHz, $\left.\mathrm{CDCl}_{3}\right) \delta 0.06(\mathrm{~s}, 6 \mathrm{H}), 0.07(\mathrm{~s}, 3 \mathrm{H}), 0.13(\mathrm{~s}, 3 \mathrm{H}), 0.89(\mathrm{~s}, 9 \mathrm{H})$, $0.90(\mathrm{~s}, 9 \mathrm{H}), 1.10(\mathrm{~s}, 3 \mathrm{H}), 1.11(\mathrm{~s}, 3 \mathrm{H}), 0.85-2.05(\mathrm{~m}, 16 \mathrm{H}), 3.11-3.17(\mathrm{~m}, 1 \mathrm{H}), 3.21-3.29(\mathrm{~m}, 1 \mathrm{H})$, 3.48-3.56 (m, 1H), 3.82-3.95 (m, 4H), $4.50(\mathrm{br}, 1 \mathrm{H})$; LRMS m/z $633.3\left[(\mathrm{M}+\mathrm{Na})^{+}\right.$; calcd for $\left.\mathrm{C}_{33} \mathrm{H}_{62} \mathrm{O}_{6} \mathrm{NaSi}_{2}: 634.0\right]$.

Compound 41: A mixture of 40 (350 mg, $0.57 \mathrm{mmol})$ in $\mathrm{AcOH}-\mathrm{H}_{2} \mathrm{O}(4: 1,15 \mathrm{~mL})$ was stirred at room temperature for $2 \mathrm{~h}$. The solvent was evaporated in vacuo and the resulting was treated wit $\mathrm{Et}_{2} \mathrm{O}$ to give crude 3,6,7-OH steroid $(280 \mathrm{mg})$ as white solid, which was used for next step without further purification. ${ }^{1} \mathrm{H}$ NMR (400 MHz, $\left.\mathrm{CDCl}_{3}\right) \delta 0.62$ (q, J = 7.5 Hz, 6H), $0.74(\mathrm{~d}, \mathrm{~J}=10.8 \mathrm{~Hz}, 1 \mathrm{H}), 0.94$ (t, $\mathrm{J}=7.6,9 \mathrm{H}), 1.01$ (s, 3H), 1.07 (s, 3H), 1.22-2.45 (m, 15H), 3.15 (brs, 1H), 3.27 (brs, 1H), 3.69 (brs, 1H), 4.31 (brs, 1H); ${ }^{13} \mathrm{C}$ NMR $\left(100 \mathrm{MHz}, \mathrm{CDCl}_{3}\right) \delta 5.9,7.5,16.4,25.0,30.6,32.4,35.8,36.3,37.1$, $37.6,39.8,48.0,48.9,53.5,57.5,63.9,68.9,71.1,74.9,80.7,220.5$.

To a solution of crude 3,6,7-OH steroid $(274 \mathrm{mg}, \leq 0.61 \mathrm{mmol})$ and $(1 \mathrm{~S})-(+)-10$-camphorsulfonic acid (14 mg, $0.061 \mathrm{mmol})$ in DMF (5 mL), was added 2,2-dimethoxypropane (0.75 mL, $6.10 \mathrm{mmol})$. The reaction was quenched with saturated $\mathrm{NaHCO}_{3}$ after being stirred at room temperature for $6 \mathrm{~h}$. The mixture was extracted with $\mathrm{Et}_{2} \mathrm{O}(15 \mathrm{~mL}$ x 3$)$ and the combined extracts were washed with brine and dried over $\mathrm{MgSO}_{4}$. The solvent was evaporated in vacuo to give crude 6,7-isopropylidene steroid 
$\left(287 \mathrm{mg}\right.$ ) as white solid, which was used for next step without further purification. ${ }^{1} \mathrm{H}$ NMR (400 $\left.\mathrm{MHz}, \mathrm{CDCl}_{3}\right) \delta 0.64(\mathrm{q}, \mathrm{J}=7.8 \mathrm{~Hz}, 6 \mathrm{H}), 0.73(\mathrm{~d}, \mathrm{~J}=8.7 \mathrm{~Hz}, 1 \mathrm{H}), 0.95$ (t, J = 7.8 Hz, 9H), 1.09 (s, $3 \mathrm{H}), 1.13(\mathrm{~s}, 3 \mathrm{H}), 1.24-1.55(\mathrm{~m}, 4 \mathrm{H}), 1.37(\mathrm{~s}, 3 \mathrm{H}), 1.39(\mathrm{~s}, 3 \mathrm{H}), 1.71-2.25(\mathrm{~m}, 10 \mathrm{H}), 2.43(\mathrm{dd}, \mathrm{J}=$ 19.3, 8.6 Hz, 1H), 3.05 (dd, J = 9.4, $9.4 \mathrm{~Hz}, 1 \mathrm{H}), 3.29$ (dd, J = 9.9, $9.9 \mathrm{~Hz}, 1 \mathrm{H}), 3.54-3.60$ (m, 1H), 4.41 (brs, $1 \mathrm{H}) ;{ }^{13} \mathrm{C}$ NMR (100 MHz, $\left.\mathrm{CDCl}_{3}\right) \delta$ 5.4, 7.1, 15.8, 17.3, 23.5, 27.0, 27.1, 30.4, 32.2, 35.2, $35.2,36.9,37.3,39.6,46.8,52.1,57.5,68.2,70.1,77.4,86.0,109.2,219.7$.

To a suspension of $\mathrm{EtPPh}_{3} \mathrm{Br}(636 \mathrm{mg}, 1.71 \mathrm{mmol})$ in THF $(2 \mathrm{~mL})$ was added $t$-BuOK $(1.0 \mathrm{M}$ in THF, $1.6 \mathrm{~mL}$ ). The mixture was stirred at room temperature for $1 \mathrm{~h}$ to give an orange-red suspension. A solution of crude 6,7 -isopropylidene steroid $(281 \mathrm{mg}, \leq 0.57 \mathrm{mmol})$ in THF $(3.0 \mathrm{~mL})$ was added and the resultant mixture was heated to $80{ }^{\circ} \mathrm{C}$ and stirred at that temperature for $3 \mathrm{~h}$. The reaction was quenched with saturated $\mathrm{NH}_{4} \mathrm{Cl}$ and extracted with $\mathrm{Et}_{2} \mathrm{O}(50 \mathrm{~mL}$ x 3). The combined extracts were washed with brine, dried over $\mathrm{MgSO}_{4}$ and concentrated under vacuum. The crude product was purified with flash choromatography on silica gel (5:1 hexanes-EtOAc) to give 41 (181 $\mathrm{mg}, 67 \%$ over 3 steps) as pale-yellow solid. $[\alpha]_{\mathrm{D}}^{24}+52.5\left(\right.$ c $\left.1.20, \mathrm{CHCl}_{3}\right) ;{ }^{1} \mathrm{H} \mathrm{NMR}\left(400 \mathrm{MHz}, \mathrm{CDCl}_{3}\right) \delta$ 0.63-0.76 $(\mathrm{m}, 7 \mathrm{H}), 0.97(\mathrm{t}, \mathrm{J}=7.9 \mathrm{~Hz}, 9 \mathrm{H}), 1.04(\mathrm{~s}, 3 \mathrm{H}), 1.09(\mathrm{~s}, 3 \mathrm{H}), 1.37(\mathrm{~s}, 3 \mathrm{H}), 1.39(\mathrm{~s}, 3 \mathrm{H}), 1.17-1.58(\mathrm{~m}$, $6 \mathrm{H}), 1.63(\mathrm{~d}, \mathrm{~J}=7.1 \mathrm{~Hz}, 3 \mathrm{H}), 1.81-1.97(\mathrm{~m}, 5 \mathrm{H}), 2.03-2.19(\mathrm{~m}, 2 \mathrm{H}), 2.32-2.38(\mathrm{~m}, 1 \mathrm{H}), 2.48(\mathrm{dd}, \mathrm{J}=$ 13.8, 2.4 Hz, 1H), 3.00 (dd, J = 9.4, 9.4 Hz, 1H), 3.27 (dd, J = 11.0, 8.9 Hz, 1H), 3.53-3.60 (m, 1H), $4.37(\mathrm{~d}, \mathrm{~J}=2.7 \mathrm{~Hz}, 1 \mathrm{H}), 5.07$ (q, J = 7.1 Hz, 1H); ${ }^{13} \mathrm{C} \mathrm{NMR}\left(100 \mathrm{MHz}, \mathrm{CDCl}_{3}\right) \delta 5.5,7.1,13.1,17.41$, 19.2, 26.2, 27.1, 30.4, 31.2, 32.3, 35.2, 36.9, 37.2, 43.5, 45.2, 46.8, 56.9, 57.4, 68.7, 70.3, 77.5, 86.2, 108.7, 112.8, 149.9; FTIR (neat) 3392, 2954, 2876, 1456, 1369, 1234, 1161, 1138, 1094, 1052, 1014, 840, 793, $734 \mathrm{~cm}^{-1}$; HRMS (ESI) m/z 505.3704 [(M+H) ${ }^{+}$; calcd for $\mathrm{C}_{30} \mathrm{H}_{53} \mathrm{O}_{4} \mathrm{Si}:$ 505.3713].

Compound 22: ${ }^{1} \mathrm{H}$ NMR (400 MHz, $\left.\mathrm{CDCl}_{3}\right) \delta 0.11$ (s, 3H), 0.19 (s, 3H), 0.91 (s, 9H), 1.07 (s, 3H), $1.12(\mathrm{~s}, 3 \mathrm{H}), 1.38$ (s, 3H), 1.43 (s, 3H), 1.63 (d, J = 7.1 Hz, 3H), 0.70-2.61 (m, 16H), 3.02 (dd, J = 9.4, $9.4 \mathrm{~Hz}, 1 \mathrm{H}), 3.29(\mathrm{dd}, \mathrm{J}=9.9,8.8 \mathrm{~Hz}, 1 \mathrm{H}), 3.53-3.62(\mathrm{~m}, 1 \mathrm{H}), 4.37(\mathrm{br}, 1 \mathrm{H}), 5.08(\mathrm{q}, \mathrm{J}=7.1 \mathrm{~Hz}, 1 \mathrm{H})$.

Compound 42: To a solution of $41(596 \mathrm{mg}, 1.18 \mathrm{mmol})$ in $\mathrm{CH}_{2} \mathrm{Cl}_{2}(10 \mathrm{~mL})$ at room temperature, were added 2,6-lutidine $(0.42 \mathrm{~mL}, 3.54 \mathrm{mmol})$ and TBSOTf $(0.41 \mathrm{~mL}, 1.77 \mathrm{mmol})$. The mixture was stirred at room temperature for $1 \mathrm{~h}$, and then water $(10 \mathrm{~mL})$ was added and stirred for 10 minutes to quench the reaction. The organic layer was separated and the aqueous layer was extracted with $\mathrm{Et}_{2} \mathrm{O}$ (15 mL x 3). The mbined extracts were washed with brine, dried over $\mathrm{MgSO}_{4}$ and concentrated under vacuum. The crude product was purified with flash chromatography on silica gel (hexanes, 50:1 -> 
30:1 hexanes-EtOAc) to give $42(750 \mathrm{mg}, 98 \%)$ as white solid. $[\alpha]_{\mathrm{D}}^{24}+47.3\left(\right.$ c $\left.0.90, \mathrm{CHCl}_{3}\right) ;{ }^{1} \mathrm{H}$ NMR $\left(400 \mathrm{MHz}, \mathrm{CDCl}_{3}\right) \delta 0.04(\mathrm{~s}, 3 \mathrm{H}), 0.05(\mathrm{~s}, 3 \mathrm{H}), 0.63-0.73(\mathrm{~m}, 7 \mathrm{H}), 0.87(\mathrm{~s}, 9 \mathrm{H}), 0.98(\mathrm{t}, \mathrm{J}=7.9 \mathrm{~Hz}$, 9H), $1.04(\mathrm{~s}, 3 \mathrm{H}), 1.09(\mathrm{~s}, 3 \mathrm{H}), 1.19-1.27(\mathrm{~m}, 2 \mathrm{H}), 1.38(\mathrm{~s}, 3 \mathrm{H}), 1.40(\mathrm{~s}, 3 \mathrm{H}), 1.44-1.58(\mathrm{~m}, 5 \mathrm{H}), 1.65$ $(\mathrm{d}, \mathrm{J}=7.1 \mathrm{~Hz}, 3 \mathrm{H}), 1.69-1.89(\mathrm{~m}, 4 \mathrm{H}), 2.04-2.21(\mathrm{~m}, 2 \mathrm{H}), 2.33-2.40(\mathrm{~m}, 1 \mathrm{H}), 2.48(\mathrm{dd}, \mathrm{J}=13.8,2.4$ $\mathrm{Hz}, 1 \mathrm{H}), 3.00(\mathrm{dd}, \mathrm{J}=9.4,9.4 \mathrm{~Hz}, 1 \mathrm{H}), 3.25(\mathrm{dd}, \mathrm{J}=11.0,8.9 \mathrm{~Hz}, 1 \mathrm{H}), 3.52-3.56(\mathrm{~m}, 1 \mathrm{H}), 4.37(\mathrm{~d}, \mathrm{~J}$ $=2.7 \mathrm{~Hz}, 1 \mathrm{H}), 5.09(\mathrm{q}, \mathrm{J}=7.1 \mathrm{~Hz}, 1 \mathrm{H}) ;{ }^{13} \mathrm{C} \mathrm{NMR}\left(100 \mathrm{MHz}, \mathrm{CDCl}_{3}\right) \delta-4.6,-4.5,5.5,7.2,13.2,17.4$, 18.09, 19.2, 25.9, 26.2, 27.1, 27.2, 31.2, 31.3, 32.5, 35.2, 37.1, 37.2, 43.6, 45.2, 47.0, 57.0, 57.5, 68.8, 71.0, 77.7, 86.2, 108.6, 112.8, 150.0; FTIR (neat) 2954, 1734, 1699, 1684, 1652, 1558, 1540, 1506, 1456, 1234, 1096, 862, $735 \mathrm{~cm}^{-1}$; HRMS (ESI) m/z $641.4390\left[(\mathrm{M}+\mathrm{Na})^{+}\right.$; calcd for $\mathrm{C}_{36} \mathrm{H}_{66} \mathrm{O}_{4} \mathrm{NaSi}_{2}$ : 641.4397].

Compound 23: ${ }^{1} \mathrm{H}$ NMR (400 MHz, $\left.\mathrm{CDCl}_{3}\right) \delta 0.05$ (s, 3H), 0.08 (s, 3H), 0.10 (s, 3H), 0.18 (s, 3H), 0.87 (s, 9H), 0.98 (s, 9H), 1.04 (s, 3H), 1.12 (s, 3H), 1.39 (s, 3H), 1.40 (s, 3H), 1.64 (d, J = $7.1 \mathrm{~Hz}$, $3 \mathrm{H}), 0.76-2.42(\mathrm{~m}, 15 \mathrm{H}), 2.58(\mathrm{dd}, \mathrm{J}=13.9,2.6 \mathrm{~Hz}, 1 \mathrm{H}), 3.01(\mathrm{dd}, \mathrm{J}=9.4,9.4 \mathrm{~Hz}, 1 \mathrm{H}), 3.27(\mathrm{dd}, \mathrm{J}=$ 11.0, $8.9 \mathrm{~Hz}, 1 \mathrm{H}), 3.51-3.59$ (m, 1H), 4.36 (br, 1H), 5.08 (q, J = 7.1 Hz, 1H); LRMS m/z 641.6 $\left[(\mathrm{M}+\mathrm{Na})^{+}\right.$; calcd for $\mathrm{C}_{36} \mathrm{H}_{66} \mathrm{O}_{4} \mathrm{NaSi}_{2}$ : 642.1].

Compound 43: To a suspension of selenium dioxide $(2.7 \mathrm{mg}, 0.024 \mathrm{mmol})$ in $\mathrm{CH}_{2} \mathrm{Cl}_{2}(0.3 \mathrm{~mL})$ was added tert-butyl hydroperoxide (5.0-6.0 M solution in decanes, 0.02 mL). The mixture was stirred at room temperature for $0.5 \mathrm{~h}$ and then cooled to $0{ }^{\circ} \mathrm{C}$. A solution of $42(30 \mathrm{mg}, 0.049 \mathrm{mmol})$ in $\mathrm{CH}_{2} \mathrm{Cl}_{2}$ $(0.5 \mathrm{~mL})$ was added and the mixture was stirred at $0{ }^{\circ} \mathrm{C}$ for $5 \mathrm{~h}$. The reaction mixture was diluted with $\mathrm{CH}_{2} \mathrm{Cl}_{2}$ and quenched with saturated $\mathrm{Na}_{2} \mathrm{SO}_{3}$. The mixture was extracted with $\mathrm{CH}_{2} \mathrm{Cl}_{2}(10 \mathrm{~mL} \times 3)$, washed with brine and dried over $\mathrm{MgSO}_{4}$. The solvent was evaporated in vacuo and the crude product was purified with flash chromatography on silica gel (5:1 hexanes-EtOAc) to afford 43 (22 mg, 72\%) as white solid. $[\alpha]_{\mathrm{D}}^{24}+23.6\left(c 0.90, \mathrm{CHCl}_{3}\right) ;{ }^{1} \mathrm{H} \mathrm{NMR}\left(400 \mathrm{MHz}, \mathrm{CDCl}_{3}\right) \delta 0.04(\mathrm{~s}, 3 \mathrm{H})$, $0.05(\mathrm{~s}, 3 \mathrm{H}), 0.63-0.70(\mathrm{~m}, 6 \mathrm{H}), 0.80(\mathrm{~d}, \mathrm{~J}=10.5,2.9 \mathrm{~Hz}, 1 \mathrm{H}), 0.87(\mathrm{~s}, 9 \mathrm{H}), 0.98(\mathrm{t}, \mathrm{J}=7.9 \mathrm{~Hz}, 9 \mathrm{H})$, $1.05(\mathrm{~s}, 3 \mathrm{H}), 1.09$ (s, 3H), 1.26-1.32 (m, 2H), $1.38(\mathrm{~s}, 3 \mathrm{H}), 1.39$ (s, 3H), 1.46-1.93 (m, 9H), $1.74(\mathrm{~d}, \mathrm{~J}$ $=7.1 \mathrm{~Hz}, 3 \mathrm{H}), 2.07-2.14(\mathrm{~m}, 1 \mathrm{H}), 2.48(\mathrm{dd}, \mathrm{J}=13.7,1.9 \mathrm{~Hz}, 1 \mathrm{H}), 3.04(\mathrm{dd}, \mathrm{J}=9.4,9.4 \mathrm{~Hz}, 1 \mathrm{H}), 3.27$ (dd, J = 9.4, 9.4 Hz, 1H), 4.38 (d, J = 2.5 Hz, 1H), 4.43 (brs, 1H), 5.55 (q, J = 7.1 Hz, 1H); ${ }^{13} \mathrm{C} \mathrm{NMR}$ $\left(100 \mathrm{MHz}, \mathrm{CDCl}_{3}\right) \delta-4.2,-4.1,5.9,7.5,13.7,18.0,18.5,20.3,26.3,27.5,27.7,31.6,32.9,35.2,37.3$, $37.37,37.7,44.1,45.8,47.4,53.5,57.9,69.2,71.3,74.8,78.0,86.2,109.2,119.5,155.3$; FTIR (neat) 
3450, 2954, 2878,1472, 1370, 1236, 1099, 1053, 860, 836, 774, $733 \mathrm{~cm}^{-1}$; HRMS (ESI) m/z $635.4504\left[(\mathrm{M}+\mathrm{H})^{+}\right.$; calcd for $\left.\mathrm{C}_{36} \mathrm{H}_{67} \mathrm{O}_{5} \mathrm{Si}_{2}: 635.4527\right]$.

Compound 44: To a solution of $43(10.7 \mathrm{mg}, 0.017 \mathrm{mmol})$ in $\mathrm{CH}_{2} \mathrm{Cl}_{2}$, was added Dess-Martin periodinane $(10.7 \mathrm{mg}, 0.025 \mathrm{mmol})$. The resultant mixture was stirred at room temperature for $1 \mathrm{~h}$, after which 2-propanol $(0.5 \mathrm{~mL})$ was added followed by $\mathrm{Et}_{2} \mathrm{O}(10 \mathrm{~mL})$. The precipitate was filtered off through a short pad of $\mathrm{MgSO}_{4}$. The filtrate was concentrated in vacuo and purified by preparative TLC (silica gel, $25 \times 25 \mathrm{~cm}, 5: 1$ hexanes-EtOAc) to yield enone $44(10.5 \mathrm{mg}, 98 \%)$ as white solid. $[\alpha]_{\mathrm{D}}^{24}-31.6\left(c 0.90, \mathrm{CHCl}_{3}\right) ;{ }^{1} \mathrm{H}$ NMR $\left(400 \mathrm{MHz}, \mathrm{CDCl}_{3}\right) \delta 0.03(\mathrm{~s}, 3 \mathrm{H}), 0.04(\mathrm{~s}, 3 \mathrm{H}), 0.63-0.71(\mathrm{~m}$, 6H), 0.86 (brs, 10H), 0.99 (t, J = 7.9 Hz, 9H), 1.07 (s, 3H), $1.25(\mathrm{~s}, 3 \mathrm{H}), 1.29-1.31(\mathrm{~m}, 1 \mathrm{H}), 1.34(\mathrm{~s}$, $3 \mathrm{H}), 1.37$ (s, 3H), 1.47-1.55 (m, 4H), 1.69-1.87 (m, 4H), 1.83 (d, J = 7.5 Hz, 3H), 2.18-2.33 (m, 2H), 2.43-2.53 (m, 2H), 2.99 (dd, J = 9.4, 9.4 Hz, 1H), 3.28 (dd, J = 9.1, 8.8 Hz, 1H), 3.51-3.57 (m, 1H), $4.44(\mathrm{~d}, \mathrm{~J}=2.7 \mathrm{~Hz}, 1 \mathrm{H}), 6.44(\mathrm{q}, \mathrm{J}=7.5 \mathrm{~Hz}, 1 \mathrm{H}) ;{ }^{13} \mathrm{C} \mathrm{NMR}\left(100 \mathrm{MHz}, \mathrm{CDCl}_{3}\right) \delta-4.6,-4.6,5.5,7.1$, 13.1, 17.5, 18.1, 19.9, 25.8, 27.0, 27.1, 31.1, 32.5, 34.3, 36.7, 37.4, 39.8, 42.3, 44.3, 47.0, 50.6, 57.1, 68.3, 70.8, 77.5, 85.6, 109.1, 128.2, 147.5, 206.6; FTIR (neat) 2954, 1724, 1649, 1472, 1378, 1235, 1212, 1154, 1093, 1048, 1006, 861, 836, 775, $736 \mathrm{~cm}^{-1}$; HRMS (ESI) m/z $633.4351\left[(\mathrm{M}+\mathrm{H})^{+}\right.$; calcd for $\left.\mathrm{C}_{36} \mathrm{H}_{65} \mathrm{O}_{5} \mathrm{Si}_{2}: 633.4371\right]$.

Compound 25: ${ }^{1} \mathrm{H}$ NMR (400 MHz, $\left.\mathrm{CDCl}_{3}\right) \delta 0.04(\mathrm{~s}, 3 \mathrm{H}), 0.05(\mathrm{~s}, 3 \mathrm{H}), 0.12(\mathrm{~s}, 3 \mathrm{H}), 0.19(\mathrm{~s}, 3 \mathrm{H})$, $0.87(\mathrm{~s}, 9 \mathrm{H}), 0.92(\mathrm{~s}, 9 \mathrm{H}), 1.10(\mathrm{~s}, 3 \mathrm{H}), 1.28(\mathrm{~s}, 3 \mathrm{H}), 1.35$ (s, 3H), 1.38 (s, 3H), 1.82 (d, J = $7.6 \mathrm{~Hz}$, $3 \mathrm{H}), 1.02-2.62(\mathrm{~m}, 16 \mathrm{H}), 3.00(\mathrm{dd}, \mathrm{J}=9.4,9.4 \mathrm{~Hz}, 1 \mathrm{H}), 3.29$ (dd, $\mathrm{J}=9.9,9.4 \mathrm{~Hz}, 1 \mathrm{H}), 3.53-3.57$ (m, $1 \mathrm{H}), 4.44$ (br, 1H), $6.45(\mathrm{q}, \mathrm{J}=7.5 \mathrm{~Hz}, 1 \mathrm{H})$; LRMS m/z $655.7\left[(\mathrm{M}+\mathrm{Na})^{+}\right.$; calcd for $\mathrm{C}_{36} \mathrm{H}_{64} \mathrm{O}_{5} \mathrm{NaSi}_{2}$ : 656.1].

Compound 45: To a solution of vinyl iodide 29 (90 mg, $0.40 \mathrm{mmol})$ [Caution: the key for this reaction is to use freshly prepared vinyl iodide] in $\mathrm{Et}_{2} \mathrm{O}(0.6 \mathrm{~mL})$ at $-78{ }^{\circ} \mathrm{C}$, was added t-BuLi $(1.7 \mathrm{M}$ in pentane, $0.70 \mathrm{~mL}$ ). The resultant mixture was transferred via canula to a suspension of copper (I) cyanide $(18 \mathrm{mg}, 0.20 \mathrm{mmol})$ in $\mathrm{Et}_{2} \mathrm{O}(0.5 \mathrm{~mL})$ at $-40{ }^{\circ} \mathrm{C}$. The mixture was stirred at $-40{ }^{\circ} \mathrm{C}$ for $0.5 \mathrm{~h}$ to give a slightly greenish homogeneous solution. The solution was cooled to $-78{ }^{\circ} \mathrm{C}$ and to this solution was added enone $44(32 \mathrm{mg}, 0.051 \mathrm{mmol})$ in $\mathrm{Et}_{2} \mathrm{O}(1.5 \mathrm{~mL})$. The reaction mixture was stirred at $-78{ }^{\circ} \mathrm{C}$, after which saturated $\mathrm{NH}_{4} \mathrm{Cl}(2 \mathrm{~mL})$ was added to quench the reaction. The organic layer was separated and the aqueous layer was extracted with $\mathrm{Et}_{2} \mathrm{O}(10 \mathrm{~mL}$ x 3). The combined 
extracts were washed with brine, dried over $\mathrm{MgSO}_{4}$, and then concentrated in vacuo. The crude product was purified with flash chromatography on silica gel (15:1 hexanes-EtOAc) to give 45 (25 $\mathrm{mg}, 68 \%$ ) as white sticky solid. $[\alpha]_{\mathrm{D}}^{24}-28.6\left(c 0.69, \mathrm{CHCl}_{3}\right) ;{ }^{1} \mathrm{H} \mathrm{NMR}\left(400 \mathrm{MHz}, \mathrm{CDCl}_{3}\right) \delta 0.03$ (s, $3 \mathrm{H}), 0.05(\mathrm{~s}, 3 \mathrm{H}), 0.62-0.70(\mathrm{~m}, 6 \mathrm{H}), 0.81(\mathrm{~d}, \mathrm{~J}=7.4 \mathrm{~Hz}, 3 \mathrm{H}), 0.83(\mathrm{~d}, \mathrm{~J}=7.3 \mathrm{~Hz}, 1 \mathrm{H}), 0.81-1.29(\mathrm{~m}$, 3H), $0.87(\mathrm{~s}, 9 \mathrm{H}), 0.90(\mathrm{~d}, \mathrm{~J}=6.9 \mathrm{~Hz}, 3 \mathrm{H}), 0.98(\mathrm{t}, \mathrm{J}=7.9 \mathrm{~Hz}, 9 \mathrm{H}), 1.02(\mathrm{~s}, 3 \mathrm{H}), 1.06(\mathrm{~s}, 3 \mathrm{H}), 1.15(\mathrm{~d}$, $\mathrm{J}=7.1 \mathrm{~Hz}, 3 \mathrm{H}), 1.34(\mathrm{~s}, 3 \mathrm{H}), 1.36(\mathrm{~s}, 3 \mathrm{H}), 1.41-1.49(\mathrm{~m}, 5 \mathrm{H}), 1.17-1.91(\mathrm{~m}, 5 \mathrm{H}), 2.02-2.20(\mathrm{~m}, 3 \mathrm{H})$, 2.38-2.47 (m, 2H), $2.98(\mathrm{dd}, \mathrm{J}=9.3,9.3 \mathrm{~Hz}, 1 \mathrm{H}), 3.27(\mathrm{dd}, \mathrm{J}=9.4,9.4 \mathrm{~Hz}, 1 \mathrm{H}), 3.53-3.58(\mathrm{~m}, 1 \mathrm{H})$, $4.40(\mathrm{~d}, \mathrm{~J}=2.8 \mathrm{~Hz}, 1 \mathrm{H}), 5.25(\mathrm{dd}, \mathrm{J}=11.3,8.0 \mathrm{~Hz}, 1 \mathrm{H}), 5.60(\mathrm{dd}, \mathrm{J}=11.3,8.2 \mathrm{~Hz}, 1 \mathrm{H}) ;{ }^{13} \mathrm{C}$ NMR $\left(100 \mathrm{MHz}, \mathrm{CDCl}_{3}\right) \delta-4.6,-4.5,5.5,7.1,17.2,17.3,17.6,18.1,19.7,19.9,21.9,25.8,27.0,27.1,29.7$, $31.1,32.5,33.1,34.3,34.8,36.8,37.5,40.9,42.2$, 42.8, 46.4, 47.1, 51.3, 57.0, 68.0, 68.7, 70.9, 77.6, 86.0, 109.1, 133.4, 217.9; FTIR (neat) 2956, 1738, 1457, 1415, 1370, 1233, 1151, 1093, 1006, 920, 861, 837, 794, 775, 734, $\mathrm{cm}^{-1}$; HRMS (ESI) m/z $731.5455\left[(\mathrm{M}+\mathrm{H})^{+}\right.$; calcd for $\mathrm{C}_{43} \mathrm{H}_{79} \mathrm{O}_{5} \mathrm{Si}_{2}$ : $731.5466]$.

Compound 31: ${ }^{1} \mathrm{H}$ NMR (400 MHz, $\left.\mathrm{CDCl}_{3}\right) \delta 0.04(\mathrm{~s}, 3 \mathrm{H}), 0.05(\mathrm{~s}, 3 \mathrm{H}), 0.10(\mathrm{~s}, 3 \mathrm{H}), 0.15(\mathrm{~s}, 3 \mathrm{H})$, $0.81(\mathrm{~d}, \mathrm{~J}=7.0 \mathrm{~Hz}, 3 \mathrm{H}), 0.82(\mathrm{~d}, \mathrm{~J}=7.3 \mathrm{~Hz}, 3 \mathrm{H}), 0.87(\mathrm{~s}, 9 \mathrm{H}), 0.94(\mathrm{~s}, 9 \mathrm{H}), 1.06(\mathrm{~s}, 3 \mathrm{H}), 1.13$ (s, 3H), $1.14(\mathrm{~d}, \mathrm{~J}=7.1 \mathrm{~Hz}, 3 \mathrm{H}), 1.37(\mathrm{~s}, 3 \mathrm{H}), 1.38(\mathrm{~s}, 3 \mathrm{H}), 1.23-2.44(\mathrm{~m}, 21 \mathrm{H}), 2.98$ (dd, J = 9.5, $9.5 \mathrm{~Hz}$, 1H), $3.27(\mathrm{dd}, \mathrm{J}=10.8,8.9 \mathrm{~Hz}, 1 \mathrm{H}), 3.52-3.59(\mathrm{~m}, 1 \mathrm{H}), 4.39$ (br, 1H), $5.23(\mathrm{dd}, \mathrm{J}=15.2,8.4 \mathrm{~Hz}, 1 \mathrm{H})$, $5.59(\mathrm{dd}, \mathrm{J}=15.2,8.4 \mathrm{~Hz}, 1 \mathrm{H})$; LRMS m/z $753.7\left[(\mathrm{M}+\mathrm{Na})^{+}\right.$; calcd for $\mathrm{C}_{43} \mathrm{H}_{78} \mathrm{O}_{5} \mathrm{NaSi}_{2}:$ 754.2].

Compound 46: To a solution of $45(7.9 \mathrm{mg}, 0.011 \mathrm{mmol})$ in THF $(1 \mathrm{~mL})$ at $-78{ }^{\circ} \mathrm{C}$, was added $\mathrm{LiAlH}_{4}(1.0 \mathrm{M}$ in THF, $0.022 \mathrm{~mL})$. The resultant mixture was stirred at $-78{ }^{\circ} \mathrm{C}$ for $1 \mathrm{~h}$ and quenched with EtOAc. Saturated potassium sodium tartrate $(5 \mathrm{~mL})$ was added. The organic layer was separated and the aqueous layer was extracted with EtOAc $(10 \mathrm{~mL} \times 3)$. The combined organic layers were washed with brine and dried over $\mathrm{MgSO}_{4}$. The solvent was evaporated in vacuo to give crude $16-\mathrm{OH}$ steroid $(8.3 \mathrm{mg})$ as slightly yellow oil. ${ }^{1} \mathrm{H}$ NMR $\left(400 \mathrm{MHz}, \mathrm{CDCl}_{3}\right) \delta 0.00(\mathrm{~s}, 3 \mathrm{H}), 0.01(\mathrm{~s}, 3 \mathrm{H}), 0.63-$ $0.69(\mathrm{~m}, 7 \mathrm{H}), 0.79(\mathrm{~d}, \mathrm{~J}=6.6 \mathrm{~Hz}, 3 \mathrm{H}), 0.80(\mathrm{~d}, \mathrm{~J}=6.4 \mathrm{~Hz}, 3 \mathrm{H}), 0.83(\mathrm{~s}, 9 \mathrm{H}), 0.78-1.34(\mathrm{~m}, 3 \mathrm{H}), 0.90$ $(\mathrm{d}, \mathrm{J}=6.5 \mathrm{~Hz}, 3 \mathrm{H}), 0.93$ (t, J = 8.0 Hz, 9H), 1.02 (s, 3H), 1.04 (d, J = 7.0 Hz, 3H), 1.07 (s, 3H), 1.43$1.56(\mathrm{~m}, 5 \mathrm{H}), 1.66-1.90(\mathrm{~m}, 4 \mathrm{H}), 2.06-2.18(\mathrm{~m}, 3 \mathrm{H}), 2.35-2.52(\mathrm{~m}, 2 \mathrm{H}), 2.94(\mathrm{dd}, \mathrm{J}=9.9,9.9 \mathrm{~Hz}, 1 \mathrm{H})$, $3.27(\mathrm{dd}, \mathrm{J}=6.7,8.7 \mathrm{~Hz}, 1 \mathrm{H}), 3.50-3.57(\mathrm{~m}, 1 \mathrm{H}), 4.17-4.22(\mathrm{~m}, 1 \mathrm{H}), 4.31(\mathrm{~d}, \mathrm{~J}=2.4 \mathrm{~Hz}, 1 \mathrm{H}), 5.34-$ $5.51(\mathrm{~m}, 2 \mathrm{H})$. 
To a solution of crude $16-\mathrm{OH}$ steroid $(8.3 \mathrm{mg})$ and DMAP $(3 \mathrm{mg}, 0.025 \mathrm{mmol})$ in pyridine $(0.45 \mathrm{~mL})$ at $0{ }^{\circ} \mathrm{C}$, was added acetic anhydride $(0.15 \mathrm{~mL}, 1.59 \mathrm{mmol})$. The reaction mixture was stirred at $0{ }^{\circ} \mathrm{C}$ for $4 \mathrm{~h}$ and then quenched by addition of water $(2 \mathrm{~mL})$. The resultant mixture was extracted with $\mathrm{Et}_{2} \mathrm{O}(5 \mathrm{~mL} \times 3)$. The combined extracts were washed with brine and dried over $\mathrm{MgSO}_{4}$. The solvent was evaporated in vacuo to give crude 16 -OAc steroid $\left(8.3 \mathrm{mg}\right.$ ) as slightly yellow oil. ${ }^{1} \mathrm{H}$ NMR (400 $\left.\mathrm{MHz}, \mathrm{CDCl}_{3}\right) \delta 0.04(\mathrm{~s}, 3 \mathrm{H}), 0.05(\mathrm{~s}, 3 \mathrm{H}), 0.60-0.72(\mathrm{~m}, 7 \mathrm{H}), 0.78(\mathrm{~d}, \mathrm{~J}=6.8 \mathrm{~Hz}, 3 \mathrm{H}), 0.81(\mathrm{~d}, \mathrm{~J}=$ $6.8 \mathrm{~Hz}, 3 \mathrm{H}), 0.77-1.45(\mathrm{~m}, 3 \mathrm{H}), 0.86(\mathrm{~d}, \mathrm{~J}=6.7 \mathrm{~Hz}, 3 \mathrm{H}), 0.87(\mathrm{~s}, 9 \mathrm{H}), 0.97$ (t, J = 7.9 Hz, 9H), $1.03(\mathrm{~s}$, $3 \mathrm{H}), 1.05(\mathrm{~d}, \mathrm{~J}=6.7 \mathrm{~Hz}, 3 \mathrm{H}), 1.15(\mathrm{~s}, 3 \mathrm{H}), 1.35$ (s, 3H), 1.37 (s, 3H), 1.45-1.86 (m, 8H), 1.95 (s, 3H), 1.96-2.32 (m, 5H), 2.53-2.60 (m, 2H), $2.92(\mathrm{dd}, \mathrm{J}=8.9,8.9 \mathrm{~Hz}, 1 \mathrm{H}), 3.24(\mathrm{dd}, \mathrm{J}=8.8,6.5 \mathrm{~Hz}, 1 \mathrm{H})$, 3.51-3.56 (m, 1H), 4.32 (brs, 1H), 5.00-5.07 (m, 1H), 5.15-5.17 (m, 2H).

A mixture of crude 16-OAc steroid $(14.2 \mathrm{mg},<0.018 \mathrm{mmol}), 1,2$-ethanedithiol $(0.065 \mathrm{~mL}, 0.72$ mmol) and (1S)-(+)-10-camphorsulfonic acid $(0.9 \mathrm{mg}, 0.0036)$ in anhydrous $\mathrm{CHCl}_{3}(1.0 \mathrm{~mL})$ was stirred at $70{ }^{\circ} \mathrm{C}$ for $1 \mathrm{~h}$. The reaction was cooled to room temperature and quenched with saturated $\mathrm{NaHCO}_{3}$. The mixture was extracted with EtOAc ( $3 \mathrm{~mL}$ x 5$)$, and the combined extracts were washed with brine and dried over $\mathrm{MgSO}_{4}$. The solvent was evaporated under reduced pressure to give a crude oil, which was purified with flash chromatography on silica gel (3:1 hexanes-EtOAc) to afford $4 \mathbf{6}$ (10.8 mg, 73\% over 3 steps) as white sticky solid. ${ }^{1} \mathrm{H}$ NMR (400 MHz, $\left.\mathrm{CDCl}_{3}\right) \delta 0.05(\mathrm{~s}, 6 \mathrm{H}), 0.60$ $0.68(\mathrm{~m}, 7 \mathrm{H}), 0.72-1.17(\mathrm{~m}, 3 \mathrm{H}), 0.80(\mathrm{~d}, \mathrm{~J}=6.7 \mathrm{~Hz}, 3 \mathrm{H}), 0.81(\mathrm{~d}, \mathrm{~J}=6.7 \mathrm{~Hz}, 3 \mathrm{H}), 0.86(\mathrm{~d}, \mathrm{~J}=6.8 \mathrm{~Hz}$, 3H), 0.88 (s, 9H), 0.97 (t, J = 7.4 Hz, 9H), 1.01 (s, 3H), 1.06 (d, J = 6.7 Hz, 3H), 1.11 (s, 3H), 1.12$1.58(\mathrm{~m}, 6 \mathrm{H}), 1.69-1.80(\mathrm{~m}, 3 \mathrm{H}), 1.93-2.02(\mathrm{~m}, 2 \mathrm{H}), 1.97(\mathrm{~s}, 3 \mathrm{H}), 2.13-2.21(\mathrm{~m}, 2 \mathrm{H}), 2.51-2.62(\mathrm{~m}$, 2H), 3.02-3.06 (m, 1H), 3.24-3.27 (m, 1H), 3.47-3.51 (m, 1H), $4.31(\mathrm{~d}, \mathrm{~J}=2.5 \mathrm{~Hz}, 1 \mathrm{H}), 5.00-5.05(\mathrm{~m}$, 1H), 5.15-5.17 (m, 2H); ${ }^{13} \mathrm{C}$ NMR (100 MHz, $\left.\mathrm{CDCl}_{3}\right) \delta$ - 4.6, 5.5, 7.1, 15.3, 16.2, 18.0, 18.2, 19.7, 20.1, 21.0, 21.5, 25.9, 29.7, 30.3, 31.0, 32.2, 33.1, 34.3, 35.7, 37.0, 43.0, 43.2, 47.5, 48.4, 55.8, 56.9, 59.8, 68.7, 71.5, 74.7, 75.3, 80.8, 132.8, 135.2, 170.2; FTIR (neat) 2291, 2850, 1684, 1652, 1558, 1456, 1261, 1097, 909, 799, $736 \mathrm{~cm}^{-1}$; LRMS m/z 757.6 [(M+Na $)^{+}$; calcd for $\left.\mathrm{C}_{42} \mathrm{H}_{78} \mathrm{O}_{6} \mathrm{NaSi}_{2}: 758.2\right]$. Compound 34: ${ }^{1} \mathrm{H}$ NMR $\left(400 \mathrm{MHz}, \mathrm{CDCl}_{3}\right) \delta 0.05(\mathrm{~s}, 6 \mathrm{H}), 0.08(\mathrm{~s}, 3 \mathrm{H}), 0.14(\mathrm{~s}, 3 \mathrm{H}), 0.80$ (d, J = 7.5 $\mathrm{Hz}, 3 \mathrm{H}), 0.81$ (d, J = 7.5 Hz, 3H), 0.88 (d, J = 6.9 Hz, 3H), $0.90(\mathrm{~s}, 9 \mathrm{H}), 0.91(\mathrm{~s}, 9 \mathrm{H}), 1.04$ (s, 3H), $1.05(\mathrm{~d}, \mathrm{~J}=8.5 \mathrm{~Hz}, 3 \mathrm{H}), 1.15(\mathrm{~s}, 3 \mathrm{H}), 1.97(\mathrm{~s}, 3 \mathrm{H}), 0.71-2.71(\mathrm{~m}, 18 \mathrm{H}), 3.03-3.11(\mathrm{~m}, 1 \mathrm{H}), 3.23-3.31$ (m, 1H), 3.45-3.55 (m, 1H), 4.32 (br, 1H), 5.00-5.09 (m, 1H), 5.11-5.17 (m, 2H); LRMS m/z 757.7 $\left[(\mathrm{M}+\mathrm{Na})^{+}\right.$; calcd for $\mathrm{C}_{42} \mathrm{H}_{78} \mathrm{O}_{6} \mathrm{NaSi}_{2}$ : 758.2]. 
Compound 1: To a solution of $46(9.2 \mathrm{mg}, 0.013 \mathrm{mmol})$ in $\mathrm{CH}_{2} \mathrm{Cl}_{2}(1.5 \mathrm{~mL})$ at room temperature, was added Dess-Martin periodinane $(9.5 \mathrm{mg}, 0.023 \mathrm{mmol})$. The resultant mixture was stirred at room temperature for $3 \mathrm{~h}$ before being quenched with saturated $\mathrm{NaHCO}_{3}-\mathrm{Na}_{2} \mathrm{SO}_{3}(1: 1,3.0 \mathrm{~mL})$. The mixture was extracted with EtOAc $(3.0 \mathrm{~mL}$ x 5). The combined extracts were washed with brine, dried over $\mathrm{MgSO}_{4}$ and concentrated in vacuo to afford crude 6-keto-7-OH steroid $(9.2 \mathrm{mg})$ as light yellow oil, which was used for next step directly without further purification.

The crude 6-keto-7-OH steroid $(9.2 \mathrm{mg},<0.013 \mathrm{mmol})$ was treated with $1.5 \mathrm{~mL}$ of a $3.0 \mathrm{~N}$ aqueous $\mathrm{HF} / \mathrm{CH}_{3} \mathrm{CN}$ solution (prepared from $48 \%$ aqueous $\mathrm{HF}$ and $\mathrm{CH}_{3} \mathrm{CN}, 1: 9$, v/v) in a polypropylene tube. The resultant solution was stirred at room temperature for $5.5 \mathrm{~h}$, then neutralized with saturated $\mathrm{NaHCO}_{3}$, and extracted with EtOAc $(3.0 \mathrm{~mL}$ x 5). The combined extracts were washed with brine, dried over $\mathrm{MgSO}_{4}$ and concentrated in vacuo. The crude oil was purified with flash chromatography on silica gel (1:2 hexanes-EtOAc) to give compound 1 ( $4.2 \mathrm{mg}, 67 \%$ over 2 steps) as a white solid (It decomposed at $\left.250{ }^{\circ} \mathrm{C}\right)$. $[\alpha]_{\mathrm{D}}^{24}+62.2\left(\mathrm{c} 0.55, \mathrm{CHCl}_{3}\right) ;{ }^{1} \mathrm{H}$ NMR $\left(500 \mathrm{MHz}, \mathrm{CDCl}_{3}\right) \delta 0.78(\mathrm{~d}, \mathrm{~J}=6.8$ $\mathrm{Hz}, 3 \mathrm{H}), 0.82(\mathrm{~d}, \mathrm{~J}=6.9 \mathrm{~Hz}, 3 \mathrm{H}), 0.96(\mathrm{~s}, 3 \mathrm{H}), 1.00(\mathrm{~d}, \mathrm{~J}=3.3 \mathrm{~Hz}, 1 \mathrm{H}), 1.07$ (d, J = 6.8 Hz, 3H), 1.15 $(\mathrm{s}, 3 \mathrm{H}), 1.21-1.58(\mathrm{~m}, 7 \mathrm{H}), 1.63-1.71(\mathrm{~m}, 1 \mathrm{H}), 1.73-1.81(\mathrm{~m}, 1 \mathrm{H}), 1.87-1.89(\mathrm{~m}, 2 \mathrm{H}), 1.96-2.01(\mathrm{~m}$, $1 \mathrm{H}), 1.98(\mathrm{~s}, 3 \mathrm{H}), 2.11-2.19(\mathrm{~m}, 2 \mathrm{H}), 2.23-2.28(\mathrm{~m}, 1 \mathrm{H}), 2.52-2.56(\mathrm{~m}, 1 \mathrm{H}), 2.60-2.68(\mathrm{~m}, 1 \mathrm{H}), 3.58-$ $3.62(\mathrm{~m}, 1 \mathrm{H}), 3.67-3.76(\mathrm{~m}, 2 \mathrm{H}), 4.36-4.43(\mathrm{~m}, 1 \mathrm{H}), 5.08-5.14(\mathrm{~m}, 1 \mathrm{H}), 5.16-5.18(\mathrm{~m}, 2 \mathrm{H})$; ${ }^{13} \mathrm{C} \mathrm{NMR}$ $\left(125 \mathrm{MHz}, \mathrm{CDCl}_{3}\right) \delta 15.45,15.65,18.05,19.66,20.08,21.14,21.49,29.35,30.31,33.06,34.29$, $36.04,36.48,40.71,42.47,42.93,43.27,48.90,54.69,55.32,56.11,60.20,68.38,70.22,74.63,78.89$, 133.04, 134.91, 170.03, 209.66; FTIR (neat) 3484, 2938, 1712, 1695, 1456, 1374, 1269, 1114, 1021, $972 \mathrm{~cm}^{-1}$; HRMS (ESI) m/z 527.3341 [(M+Na) ${ }^{+}$; calcd for $\left.\mathrm{C}_{30} \mathrm{H}_{48} \mathrm{O}_{6} \mathrm{Na}: 527.3349\right]$. 

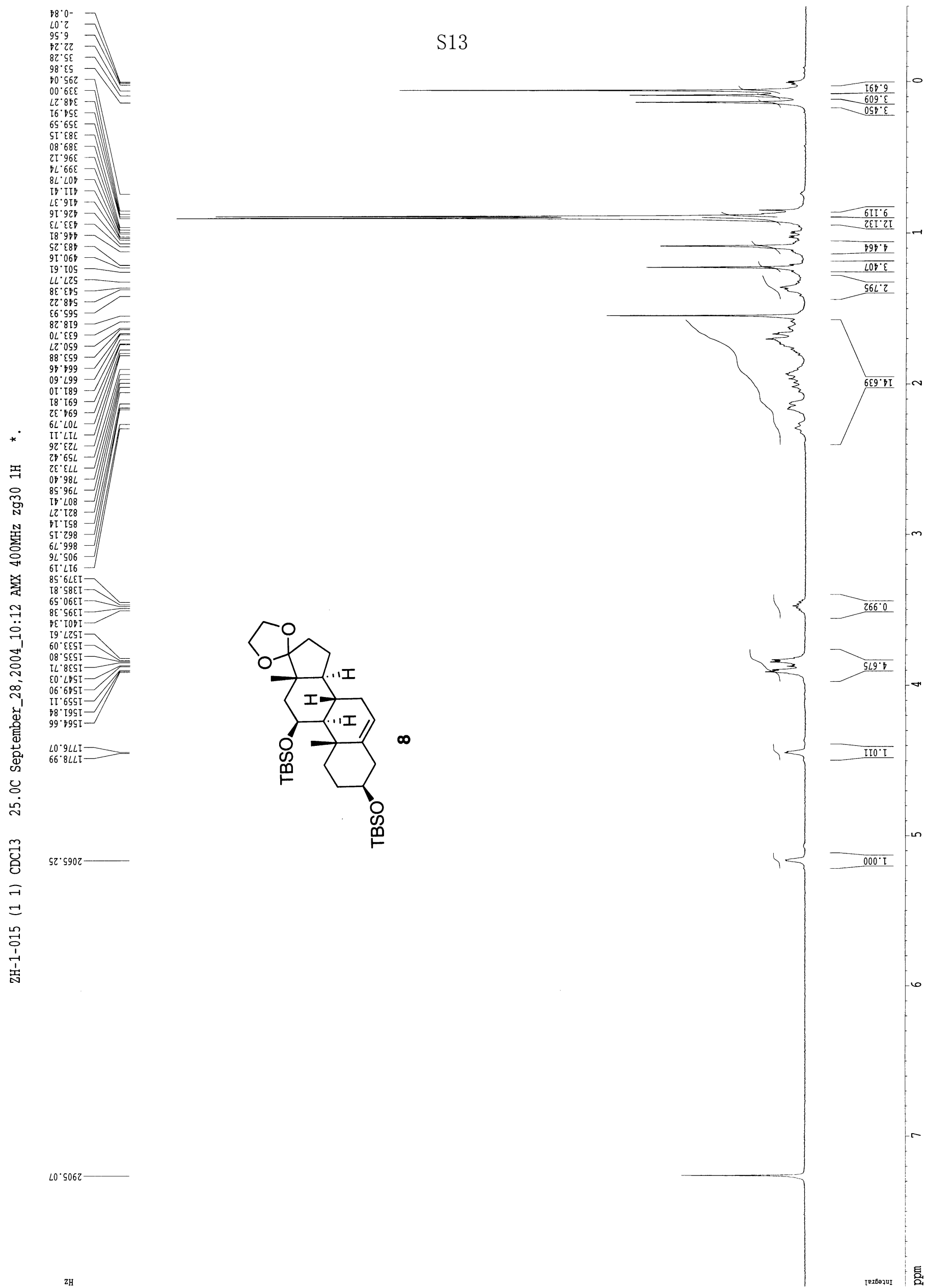


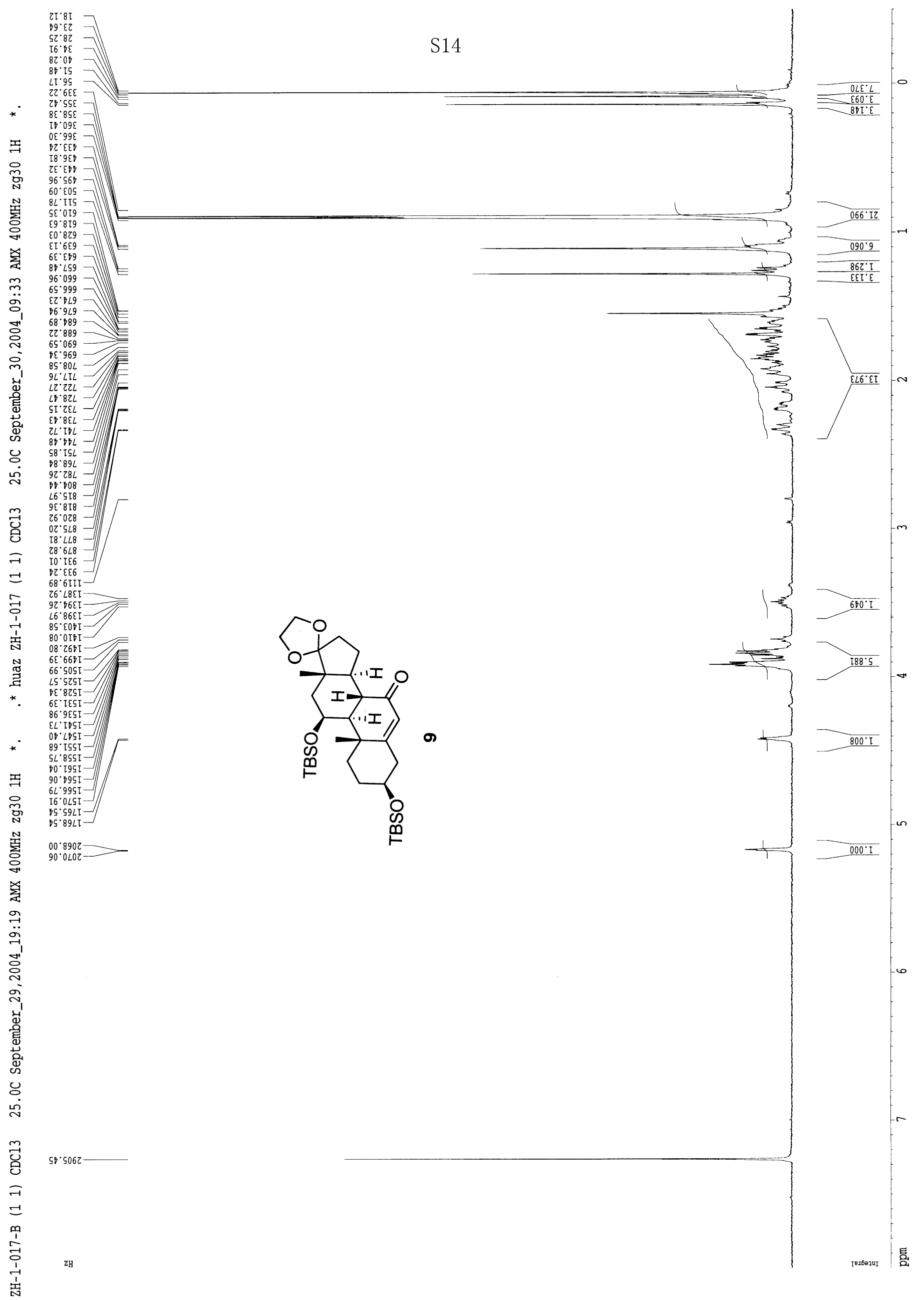



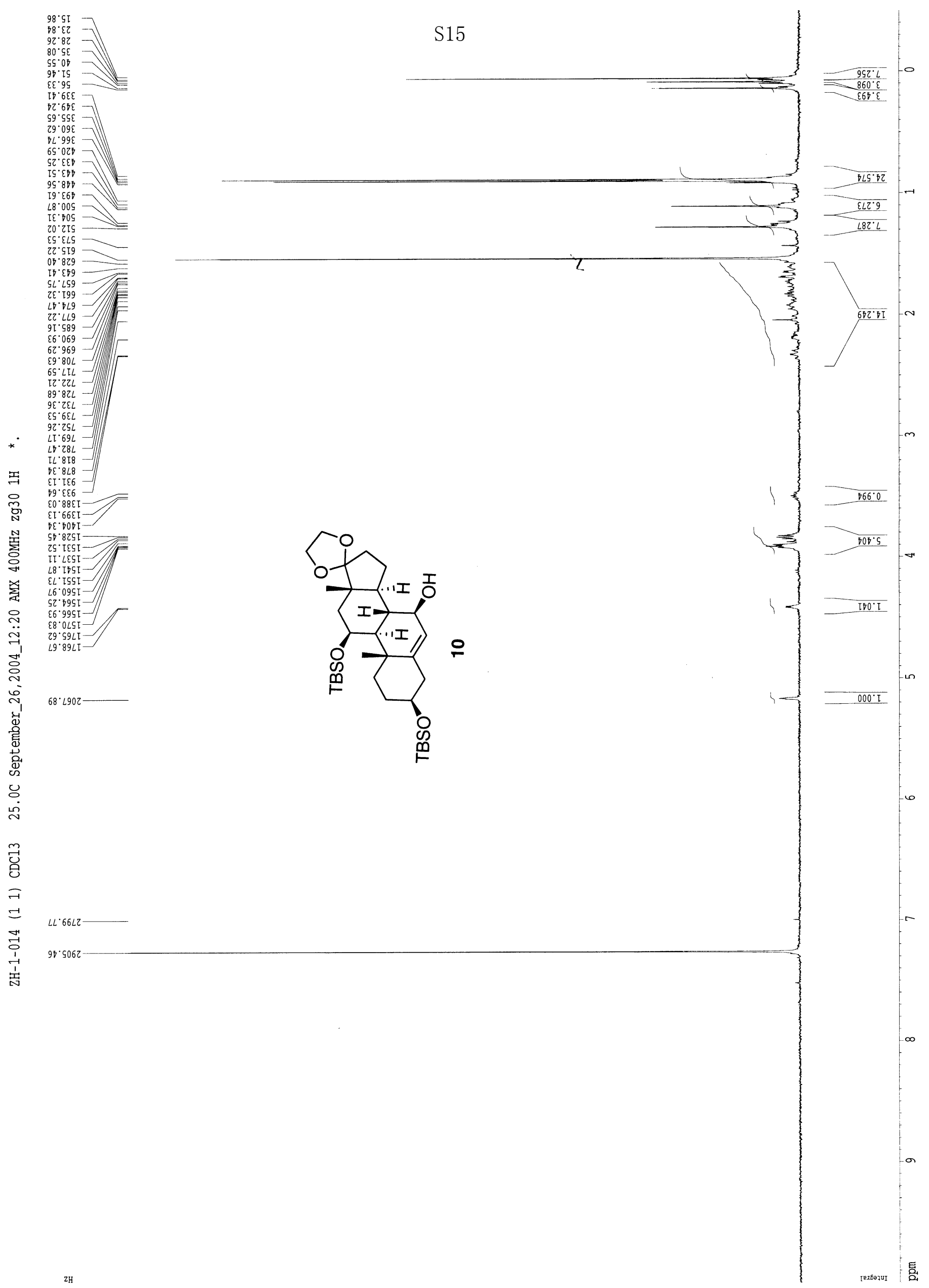


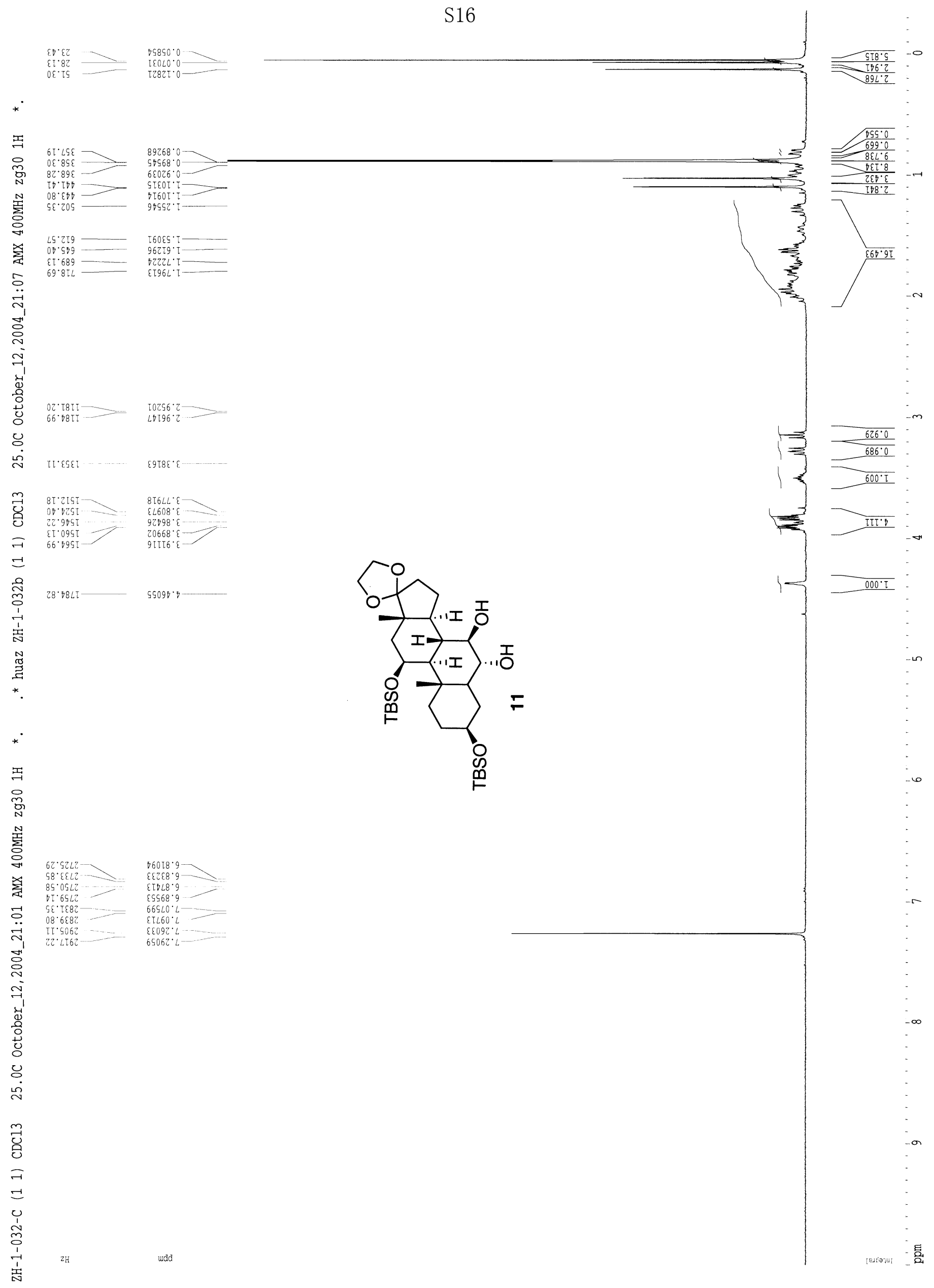



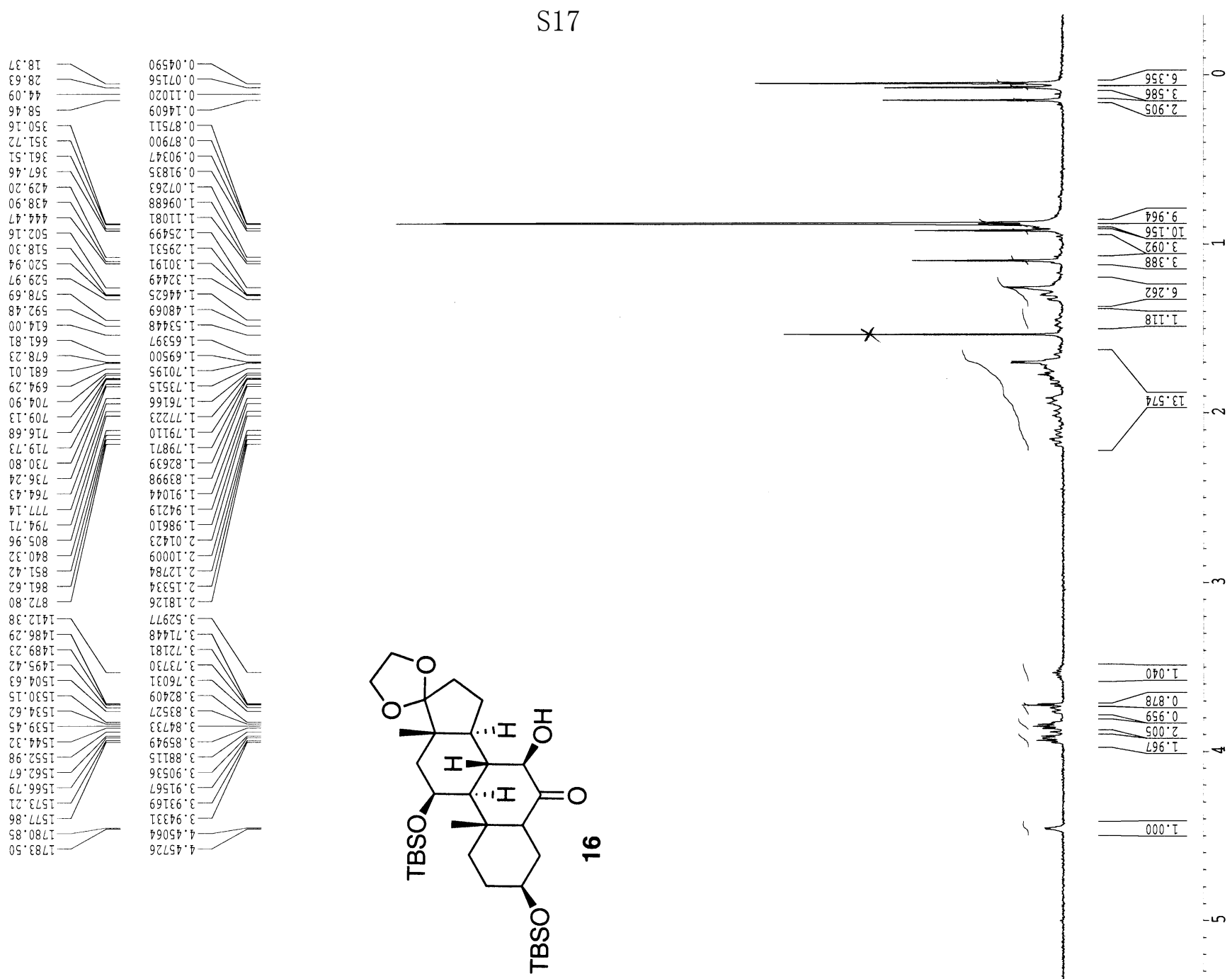

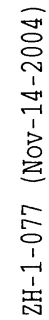

ભ 

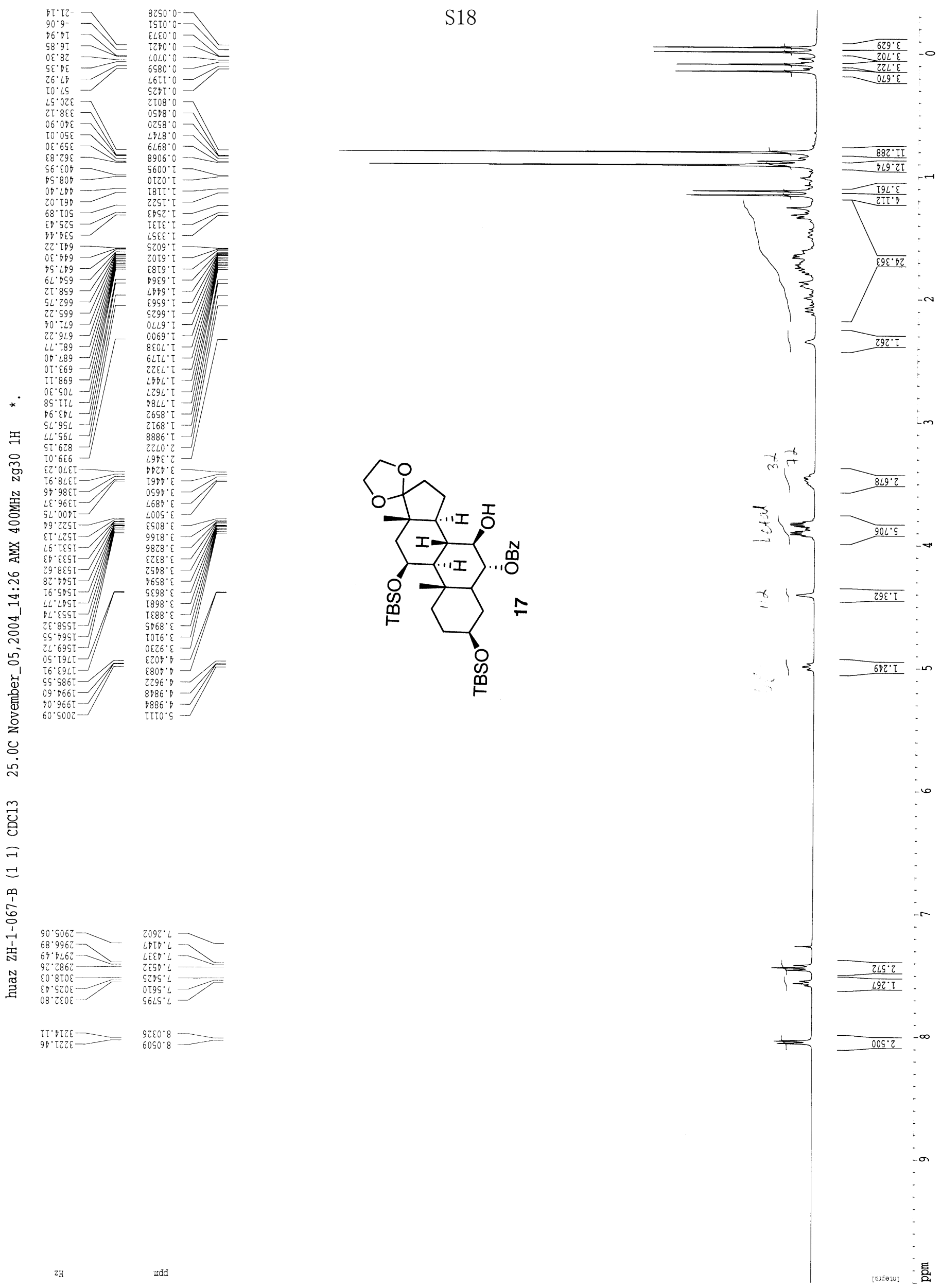

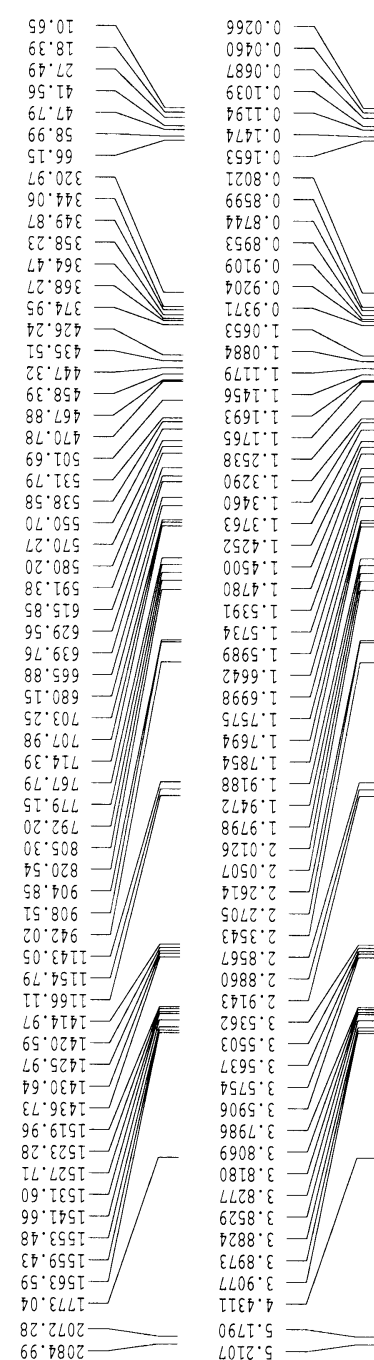

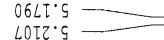

S19

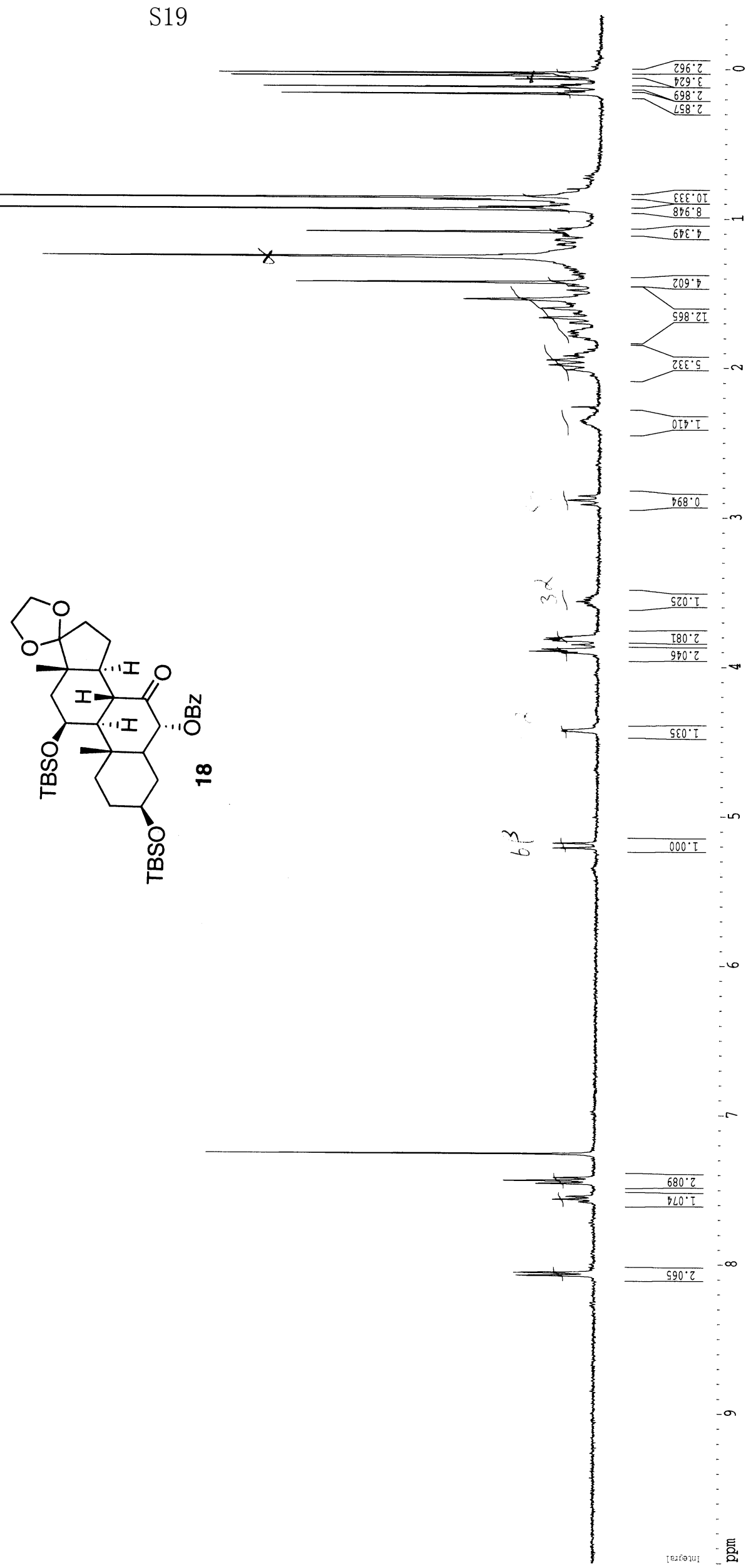

7
0
0
1
1
0
1
1
0
3
3
1
0
0
0
1
1
1

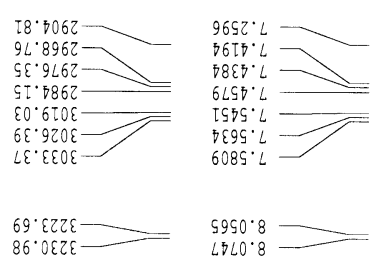




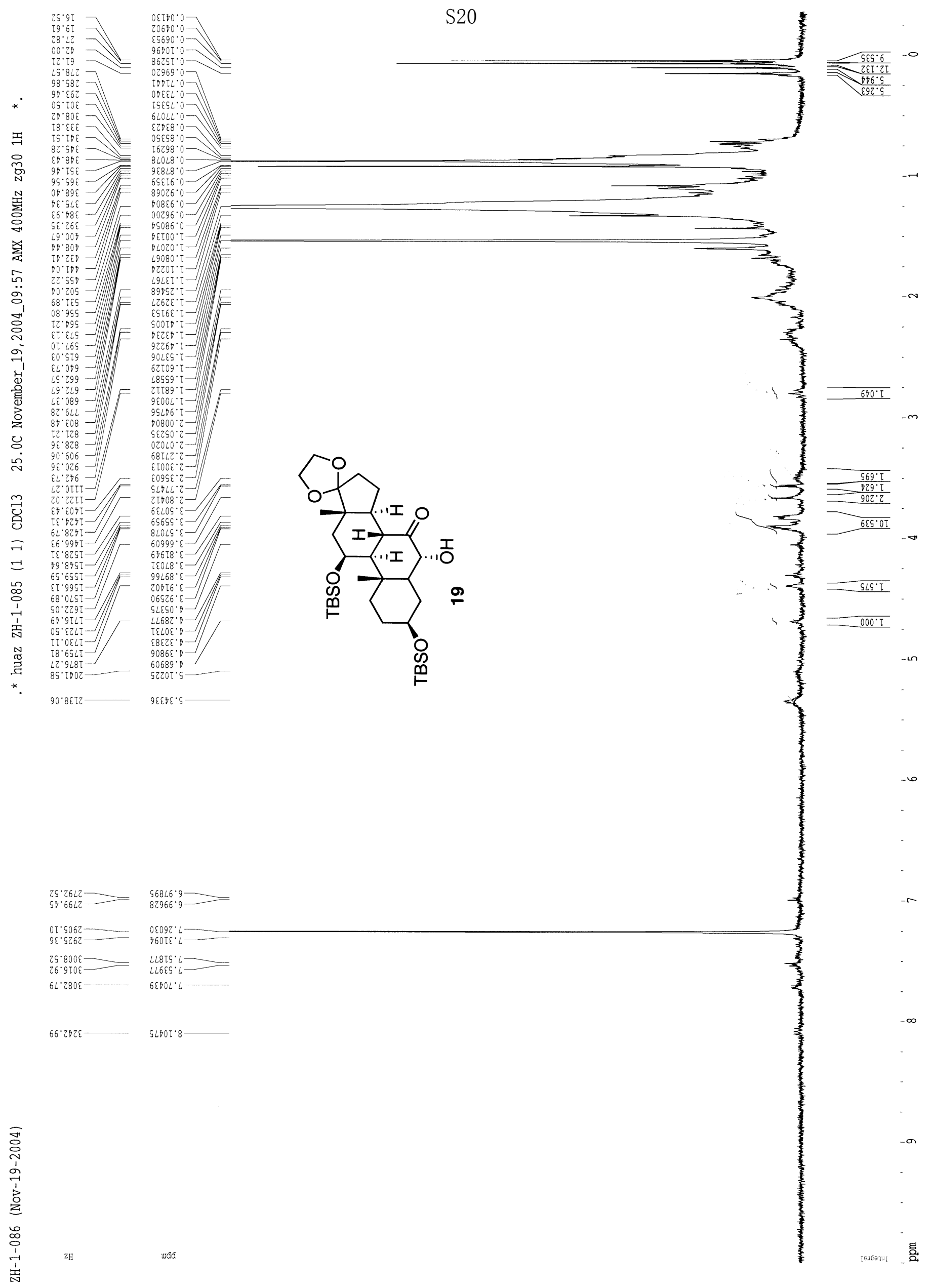




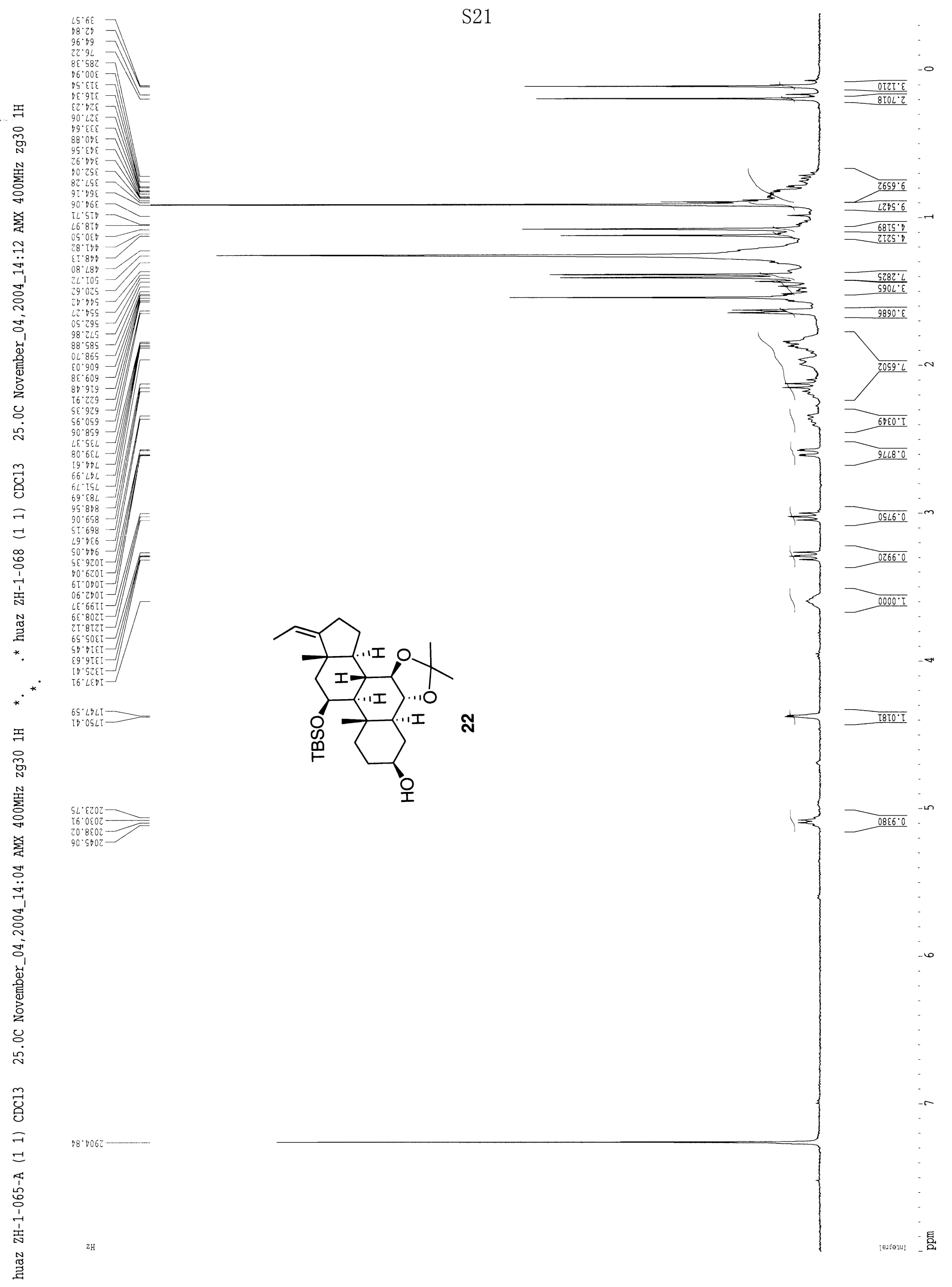




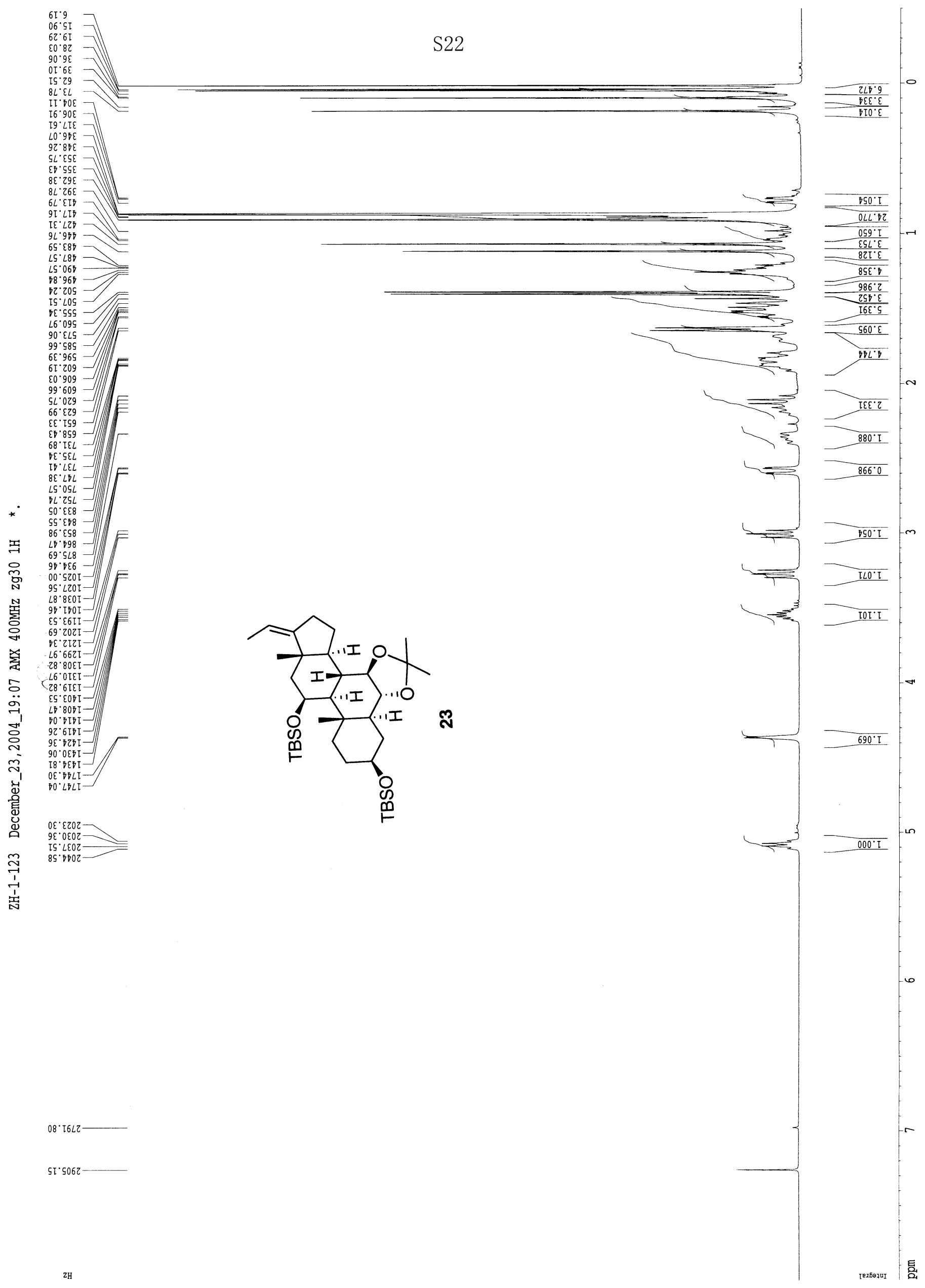




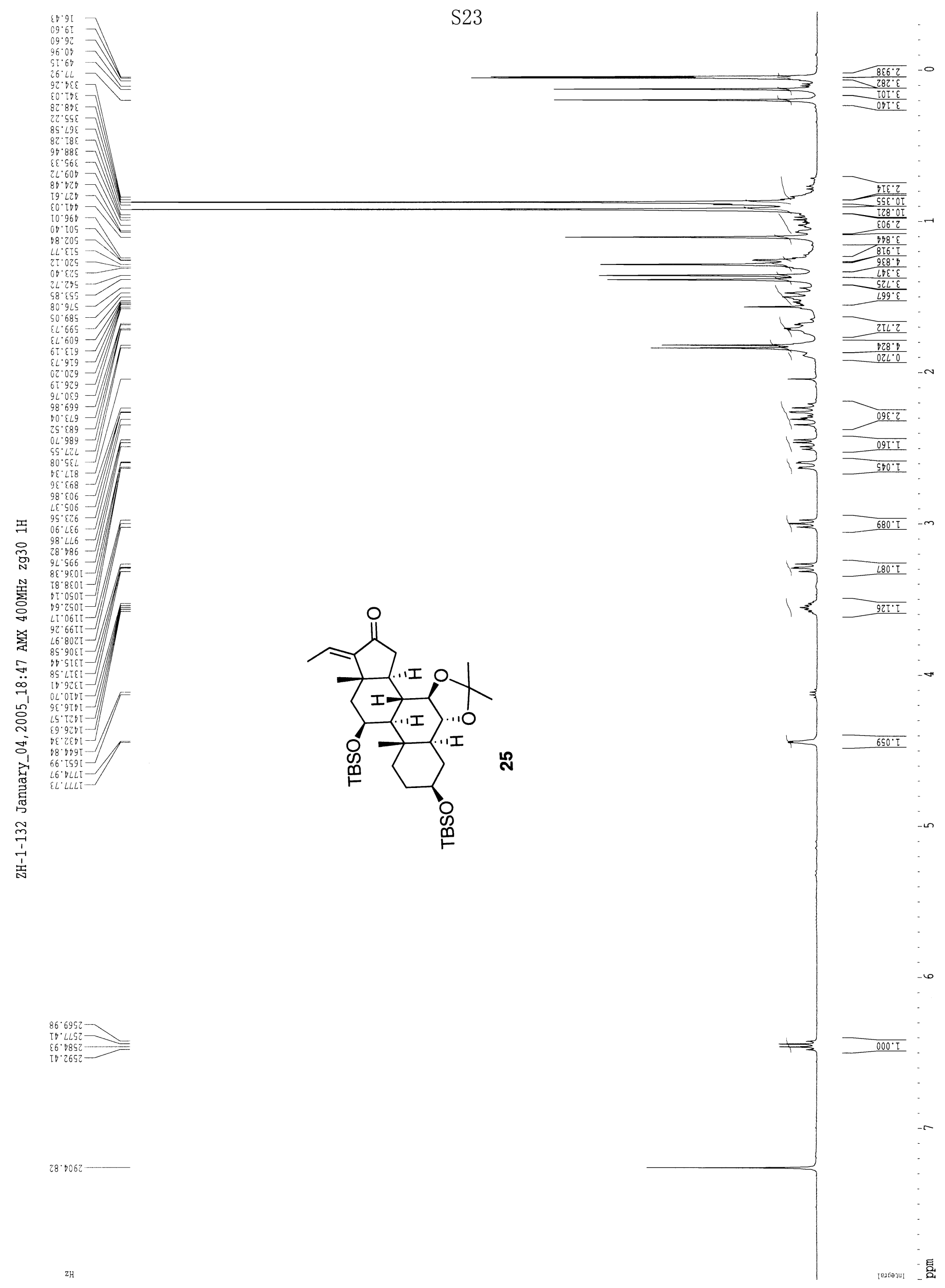




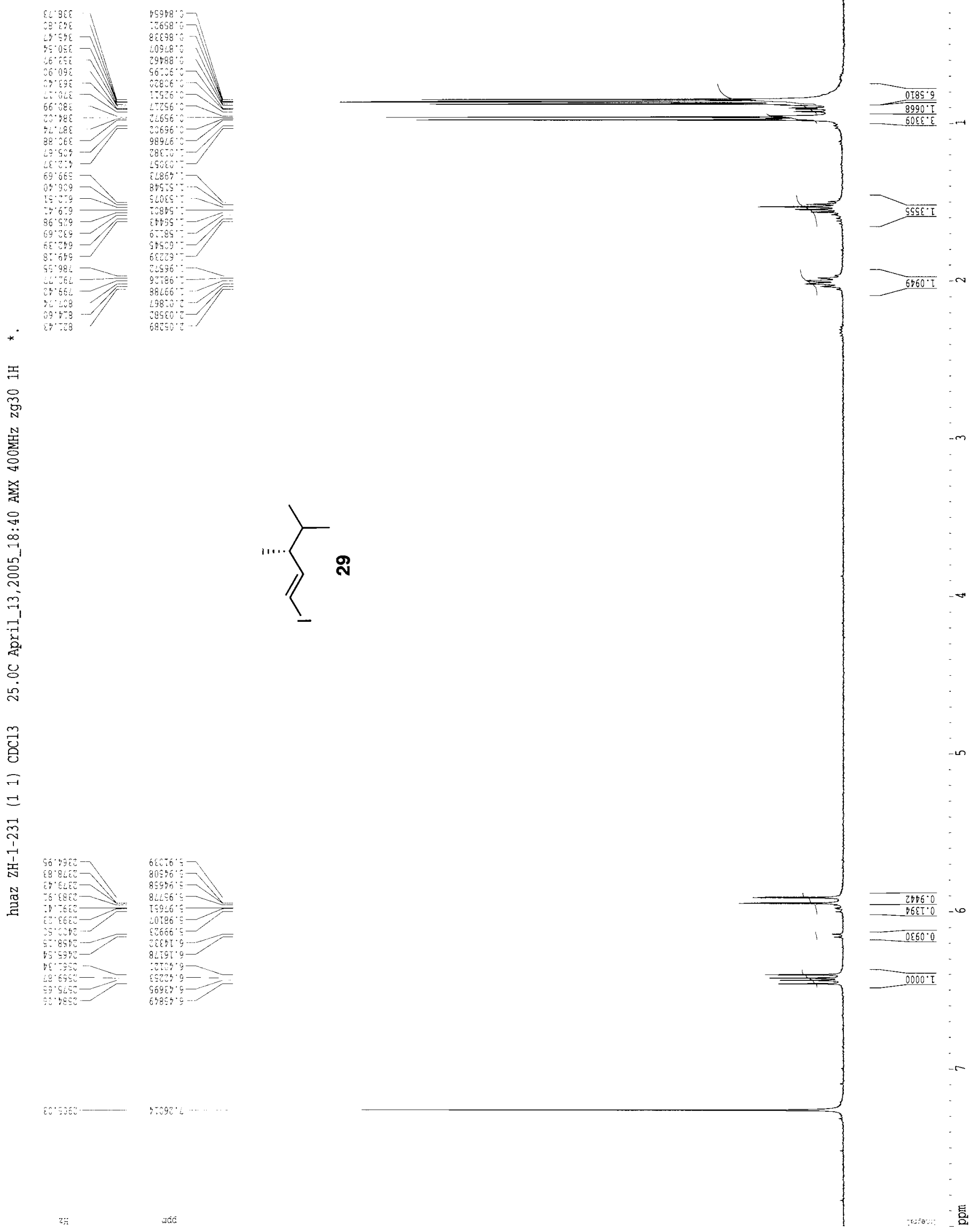



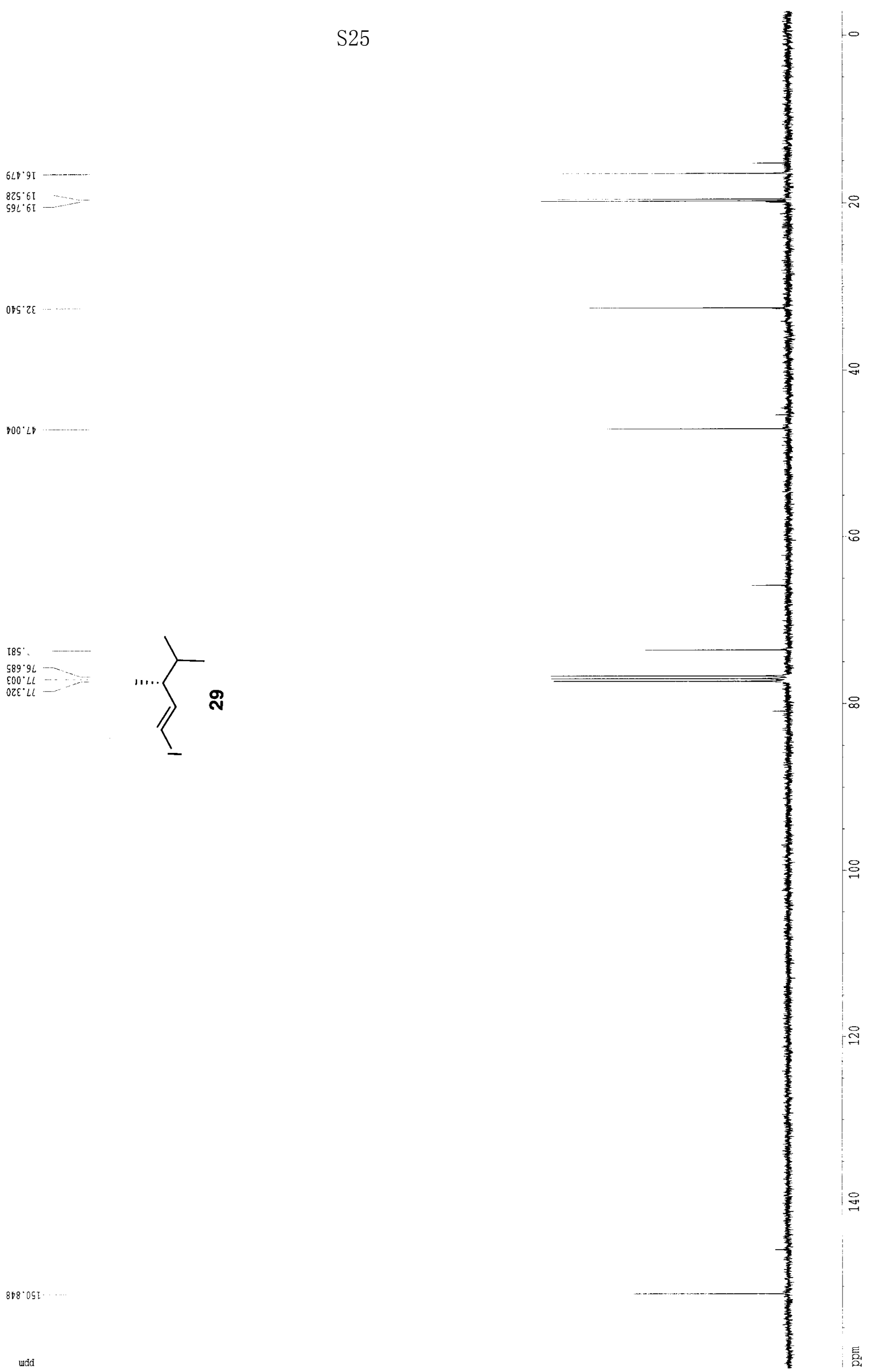


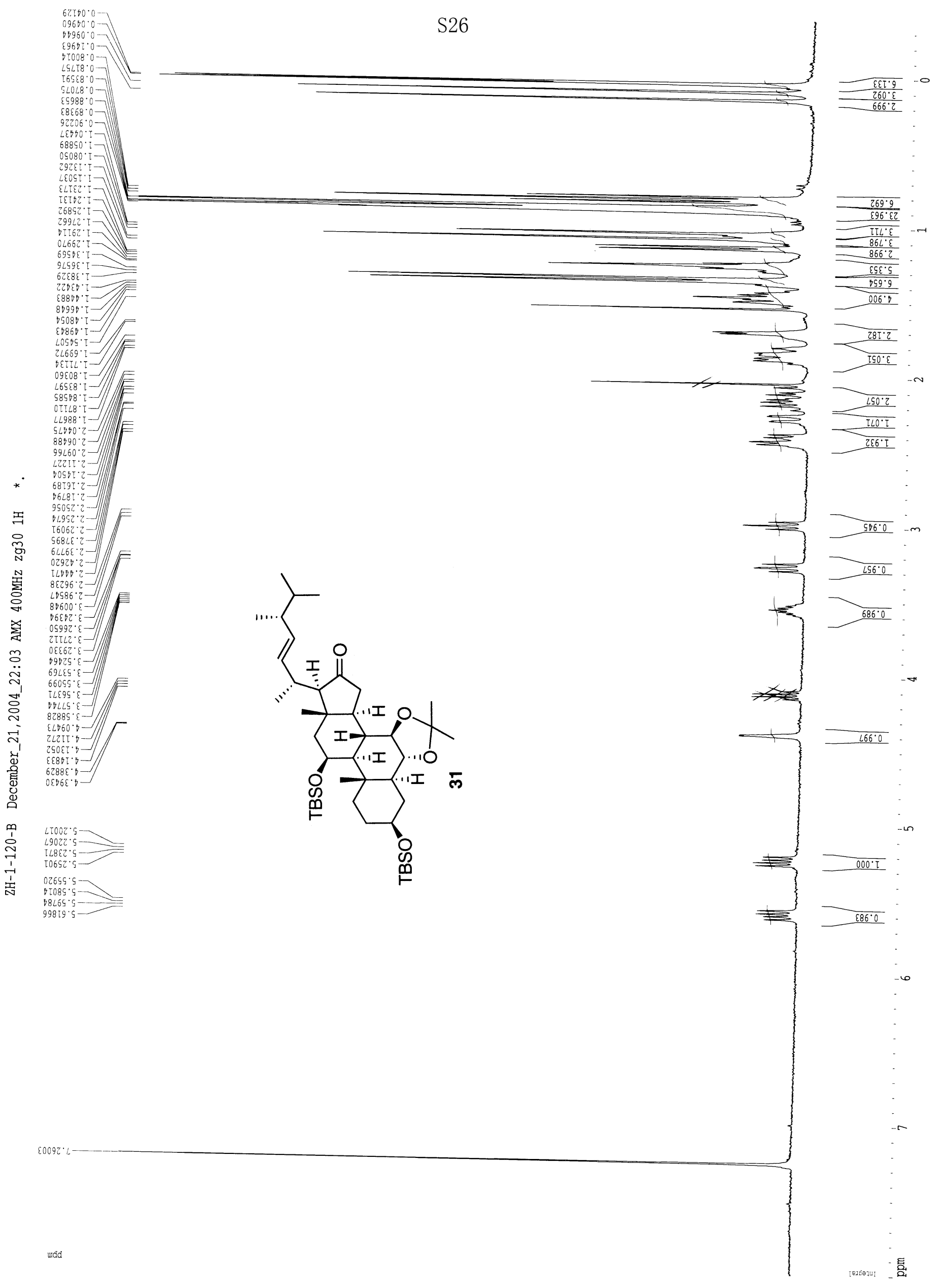




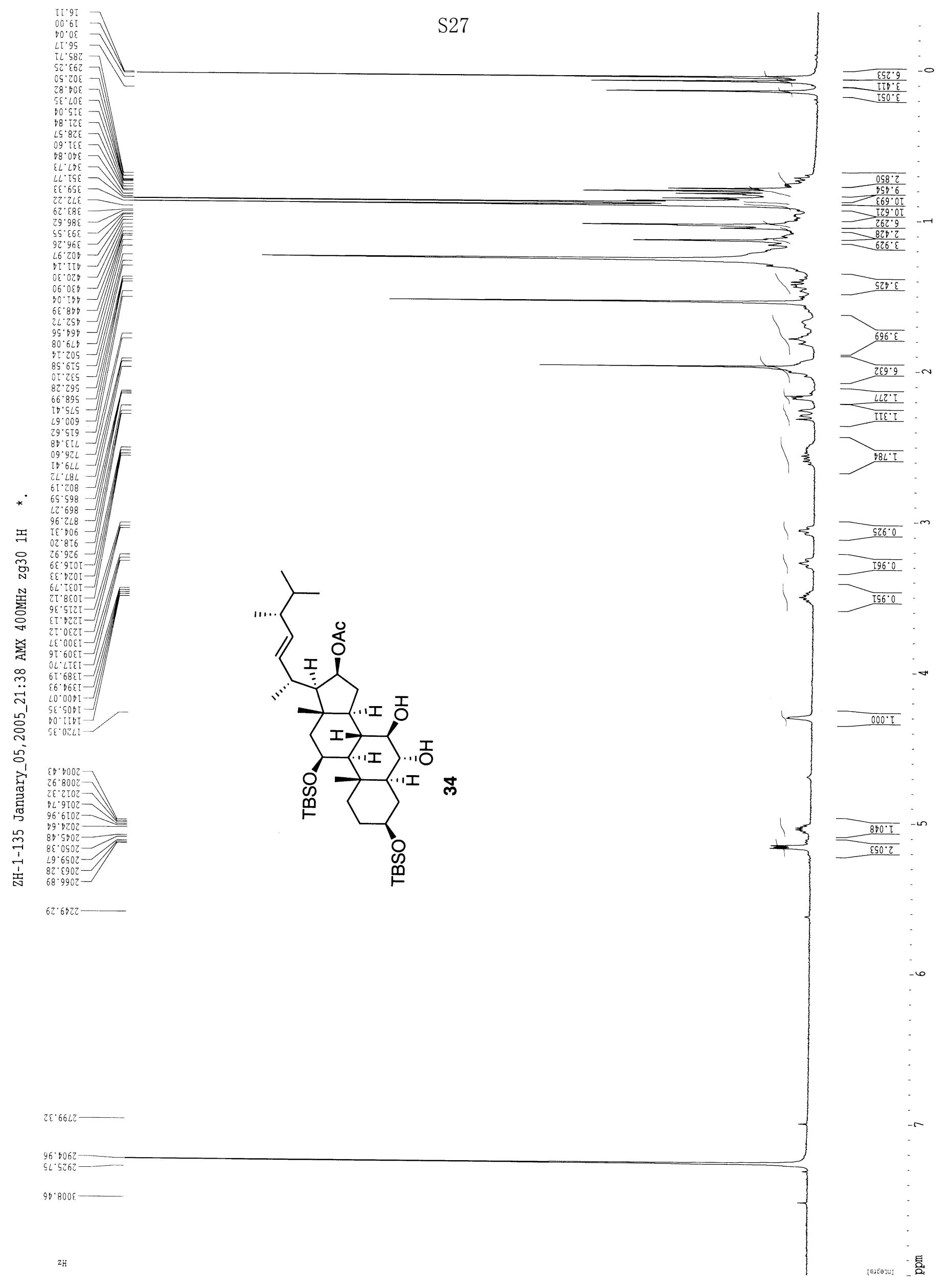




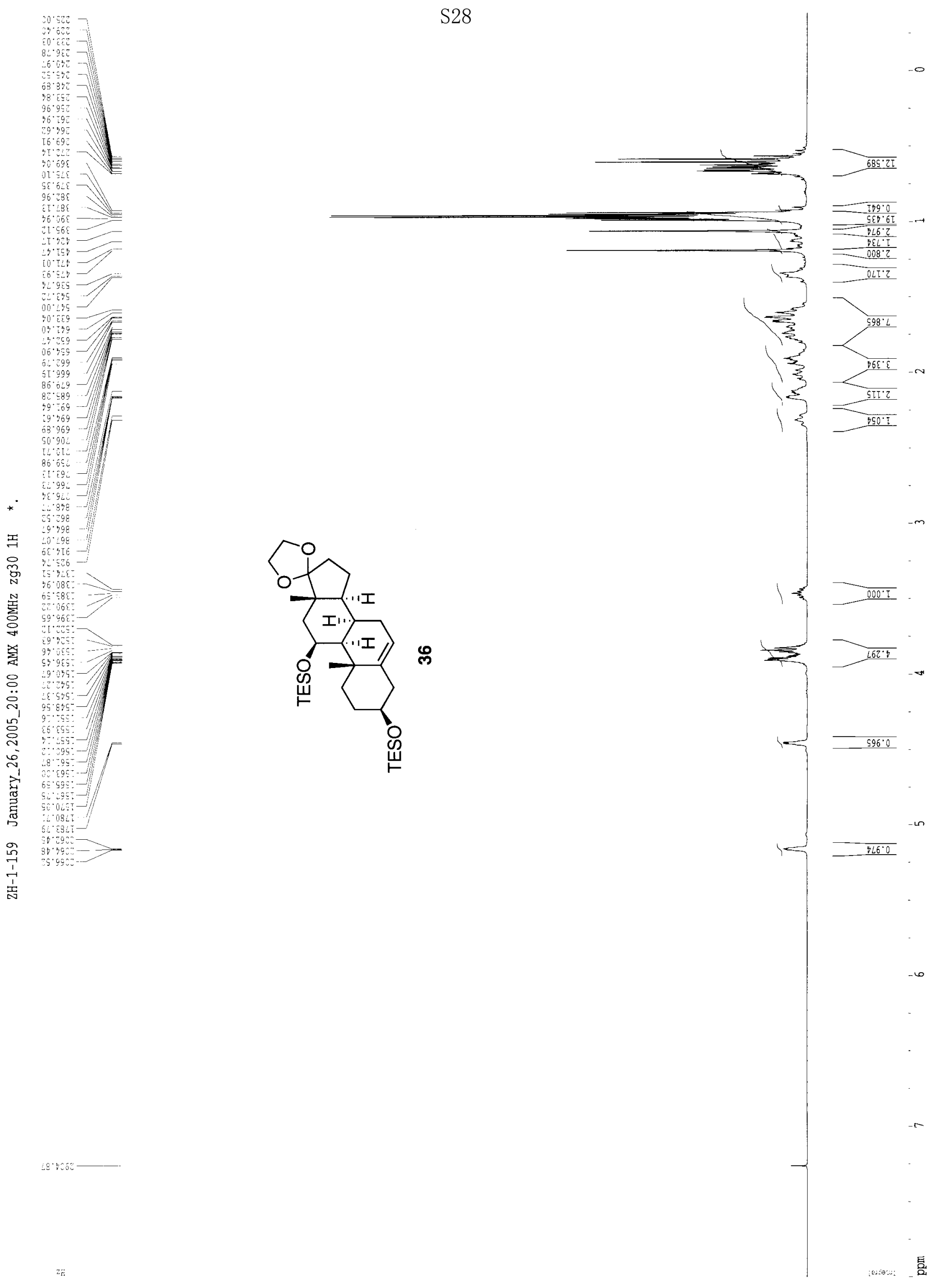




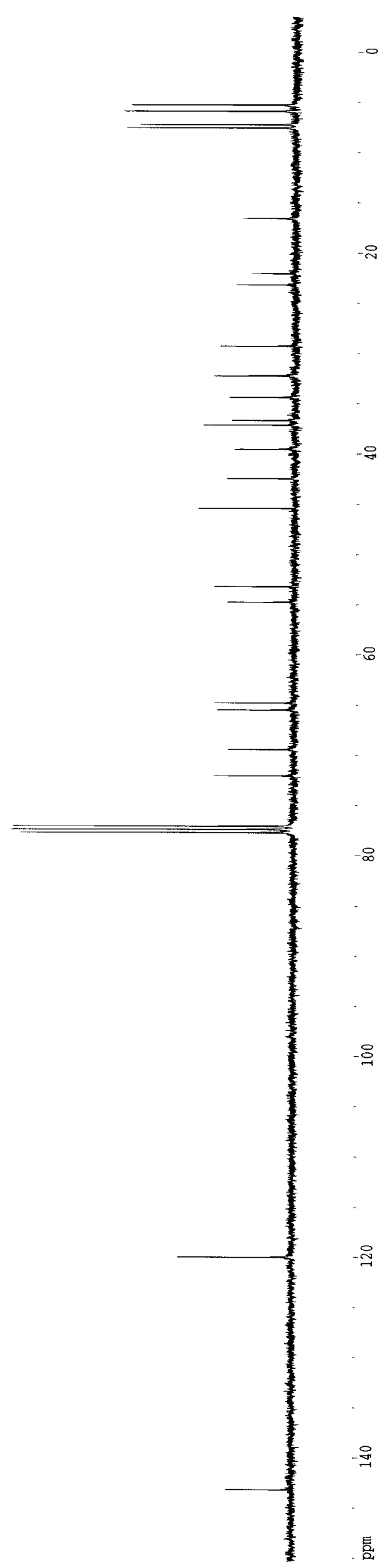




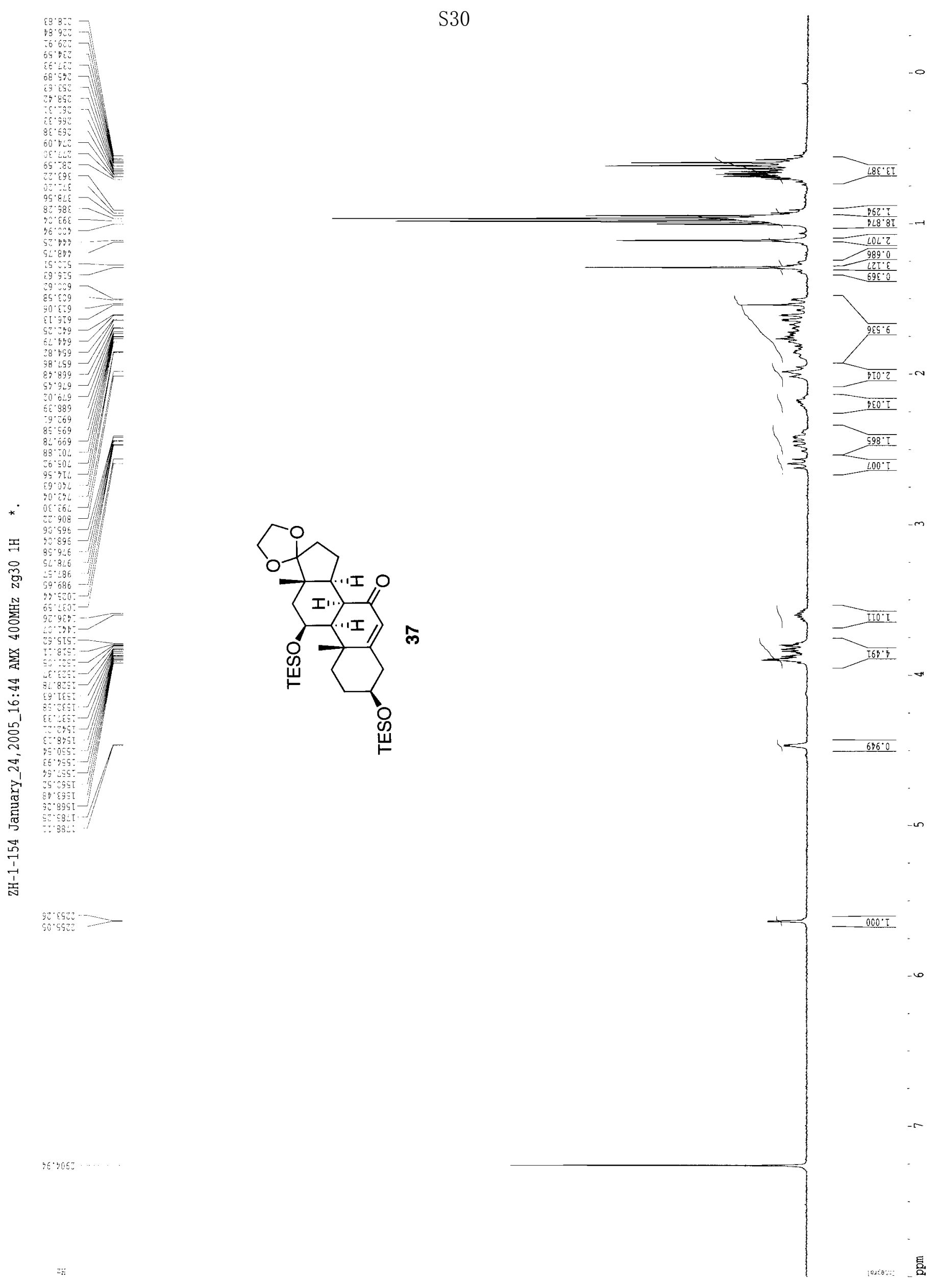


S31
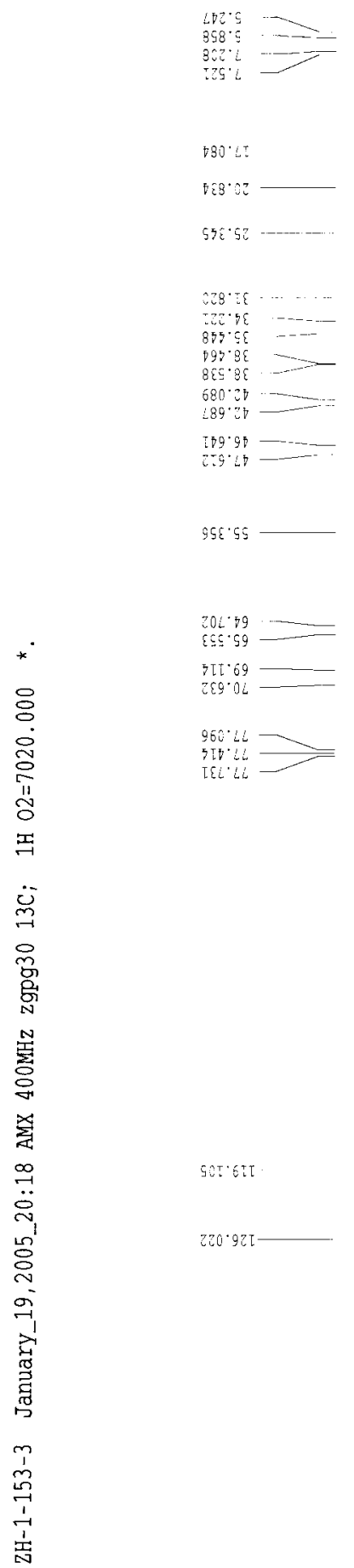

958.55
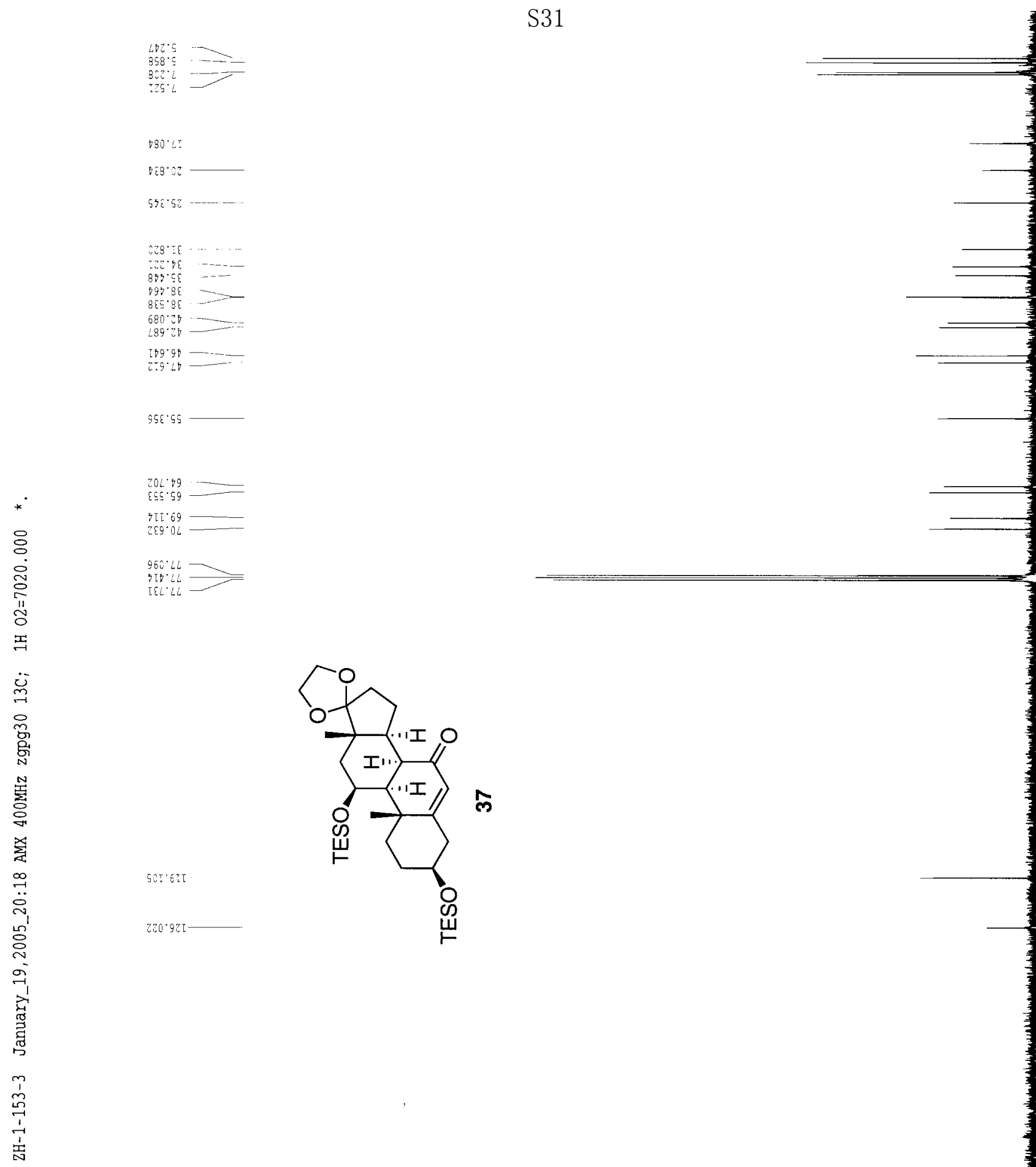

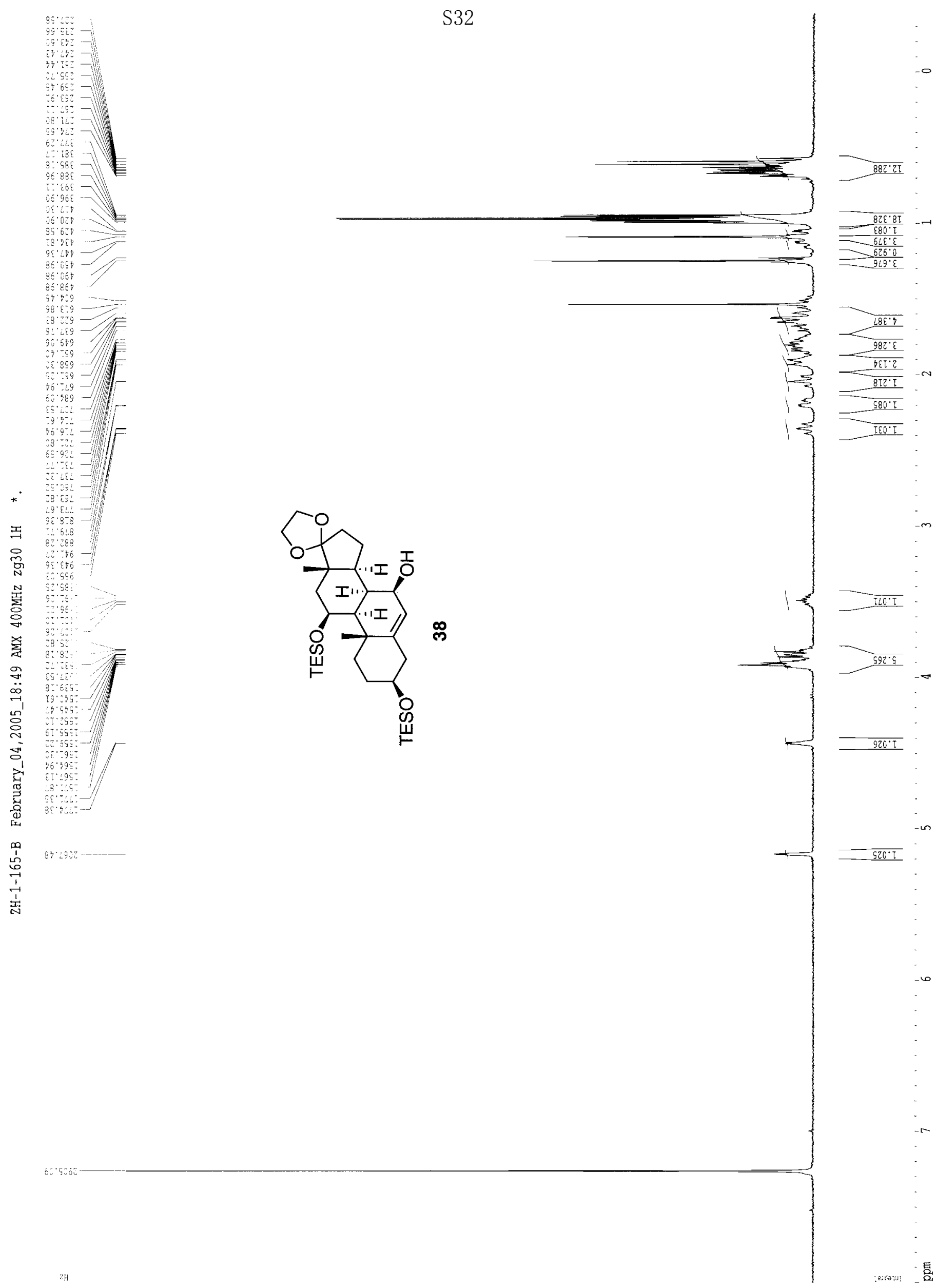

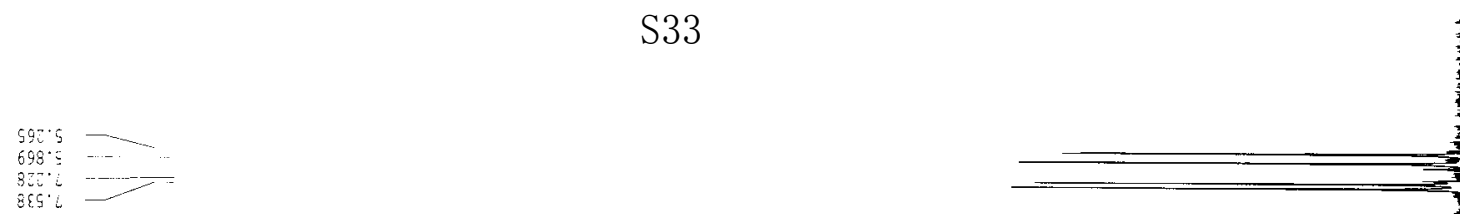

ถึต.9 —-.

ORE 28

2.8 .52

DET:DE
DED

$9+\hat{\imath} \cdot 9 \hat{\imath}$

EuT.LE

$-66 \%$

86950

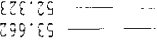

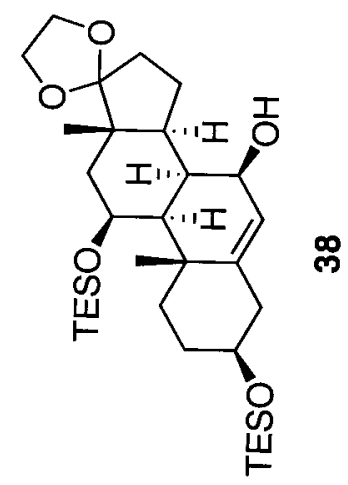

$99.09 \ldots-$
$205.55-$

sor. $08-$

$680 \cdots$

6e?

a

$y:-2$

窝

$\stackrel{8}{8}$

灵

$\stackrel{\infty}{\sharp !}$

ต

帘

官

空

劣

年

809.60

B8: $27:--$ 

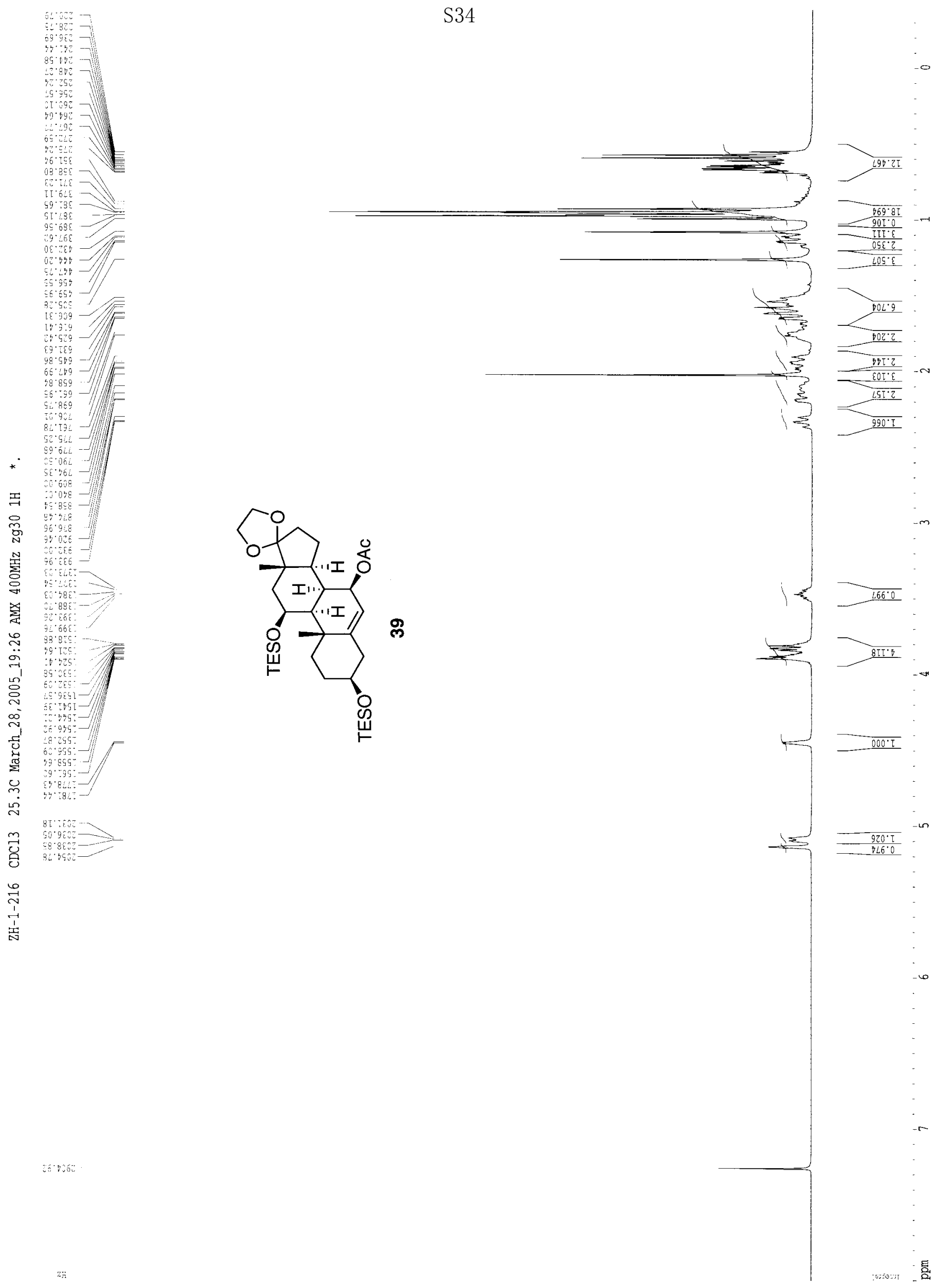
S35

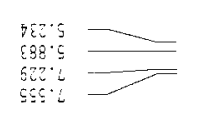

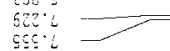

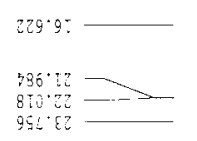

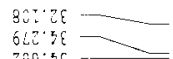

oug. is -

पye ge

Lใ6.88 - -

236.5

$*$
8
8
8
8
8
8

$\llcorner \$ 9 \cdot 9 \neq$

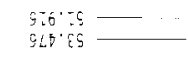

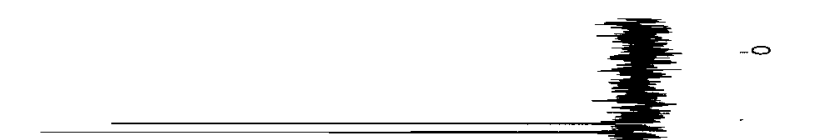

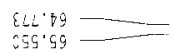

$-20.60$

g.c.

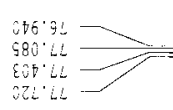

m

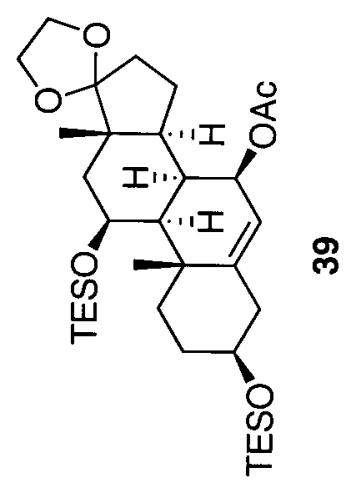

$89 E^{\circ} 6 E^{2}$

嘒

-

$\exists$

总

麄

吉

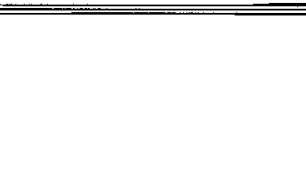



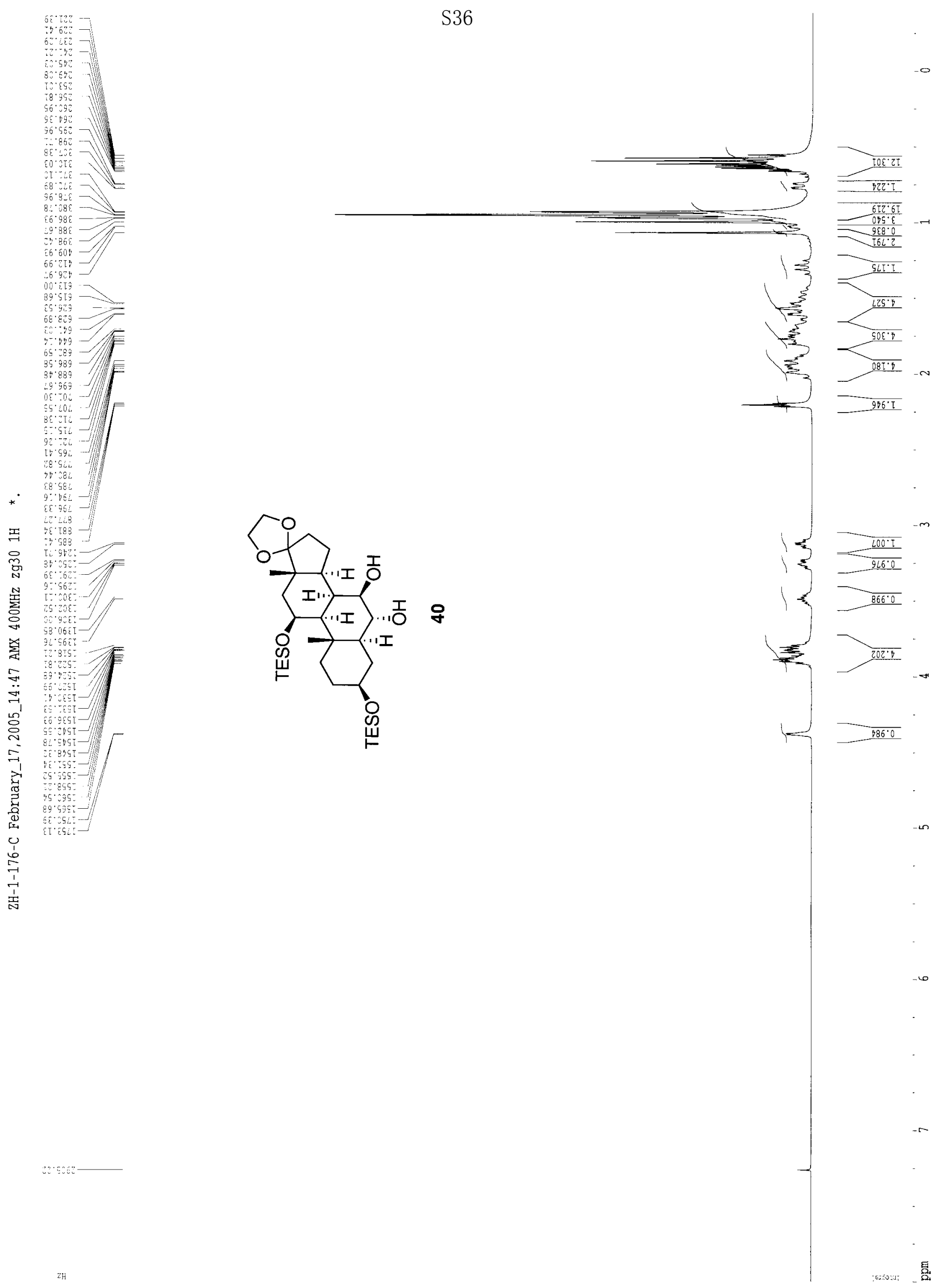
906.5
488.5
$680.2-1$
255.2

8559t

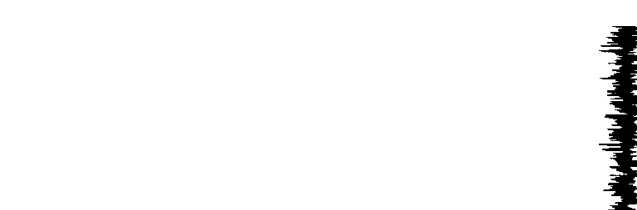

$2698 \varepsilon=$
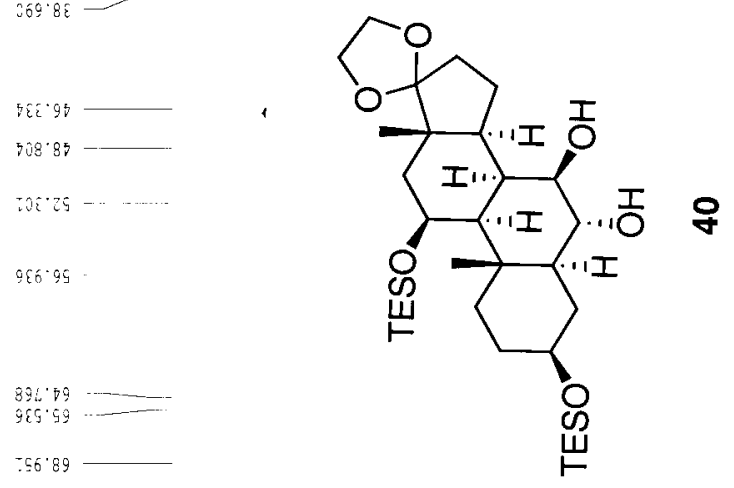

$5,88.85-$

20125-...

966.95

89.69

756.89

$589^{\circ} \mathrm{TL}$

896.52

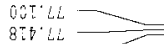

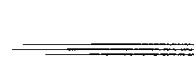

$8: 98^{\circ}+8$

$\sum_{i=1}^{x}$

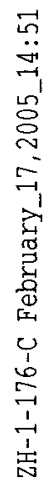




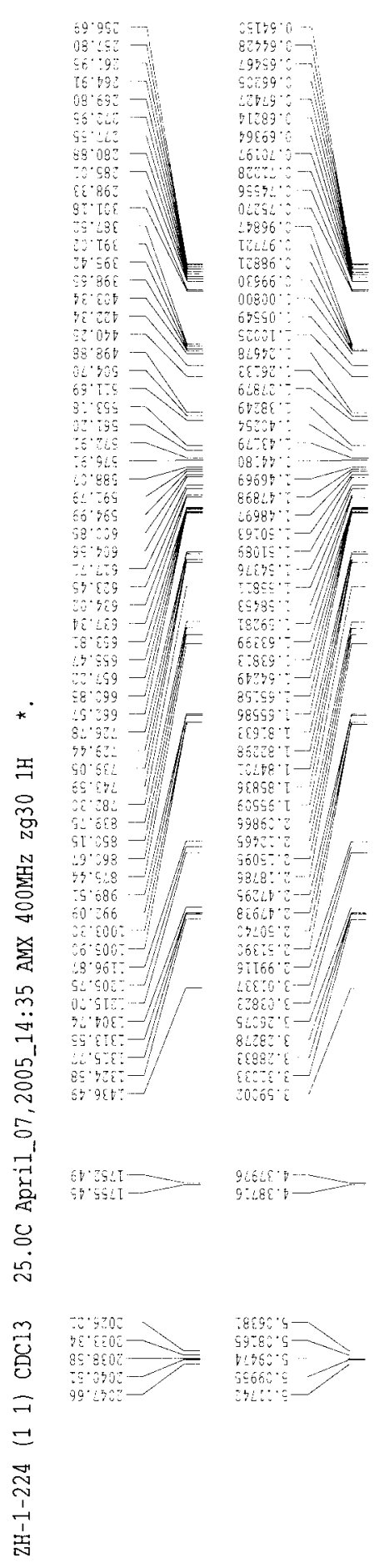

S38
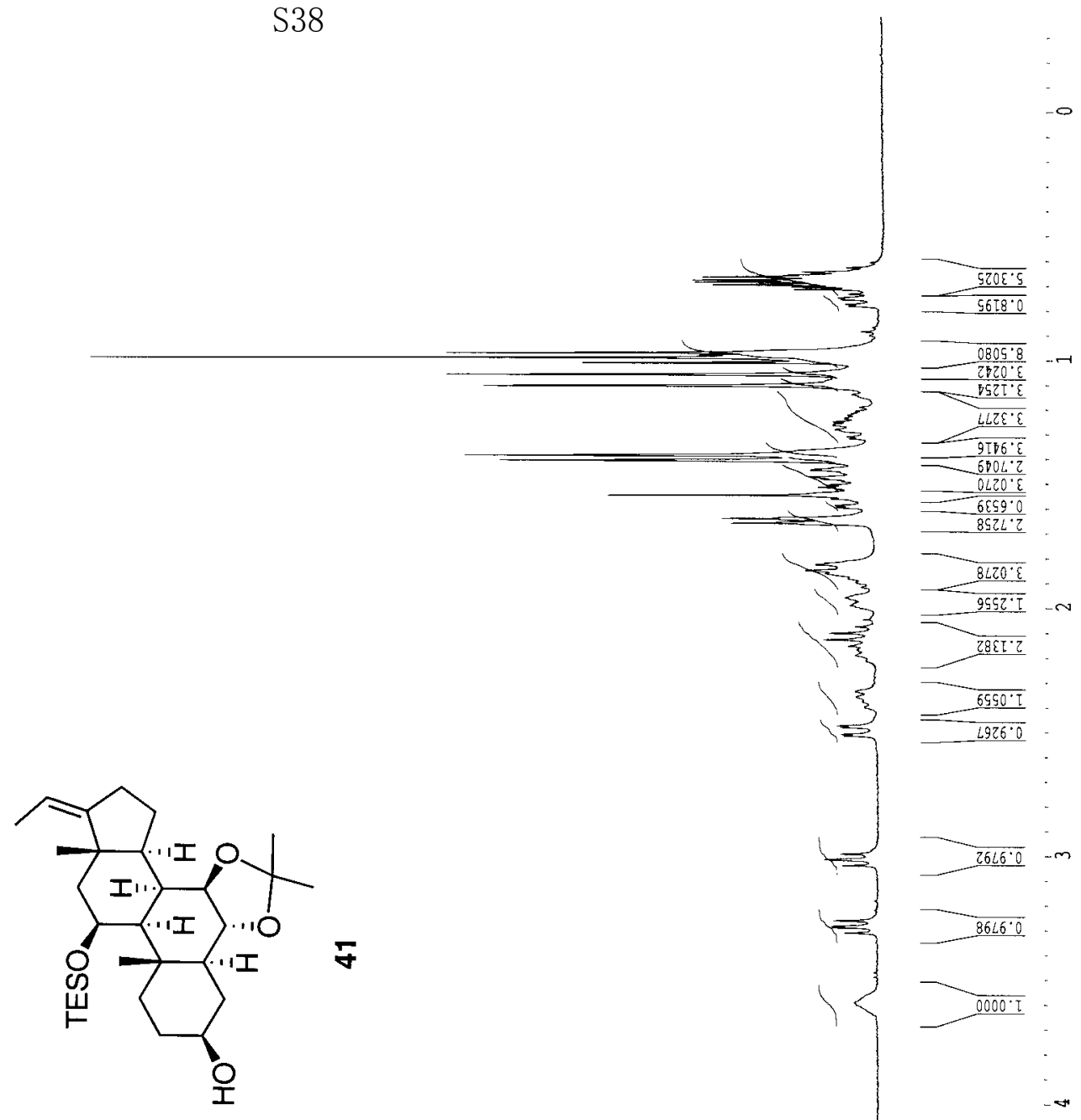


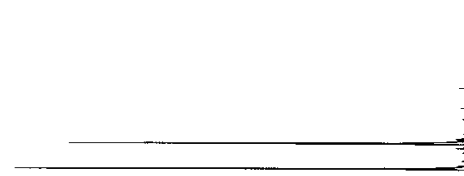

$855.8 T$

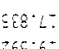

20.9. $28=$

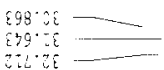

..pors

ร\& $2 \varepsilon$

Gos. 28

56058
$585: 98$
$082: 58$
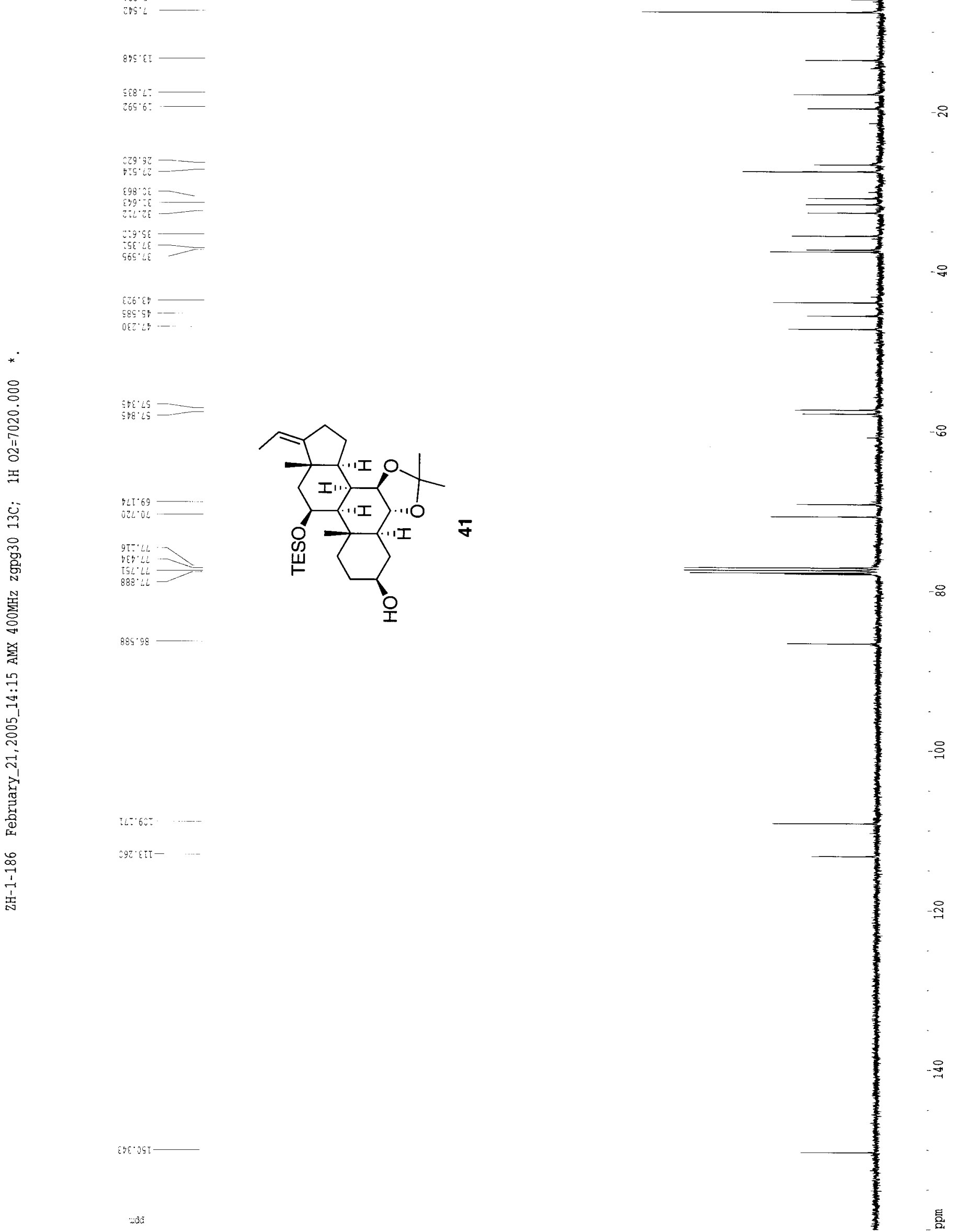

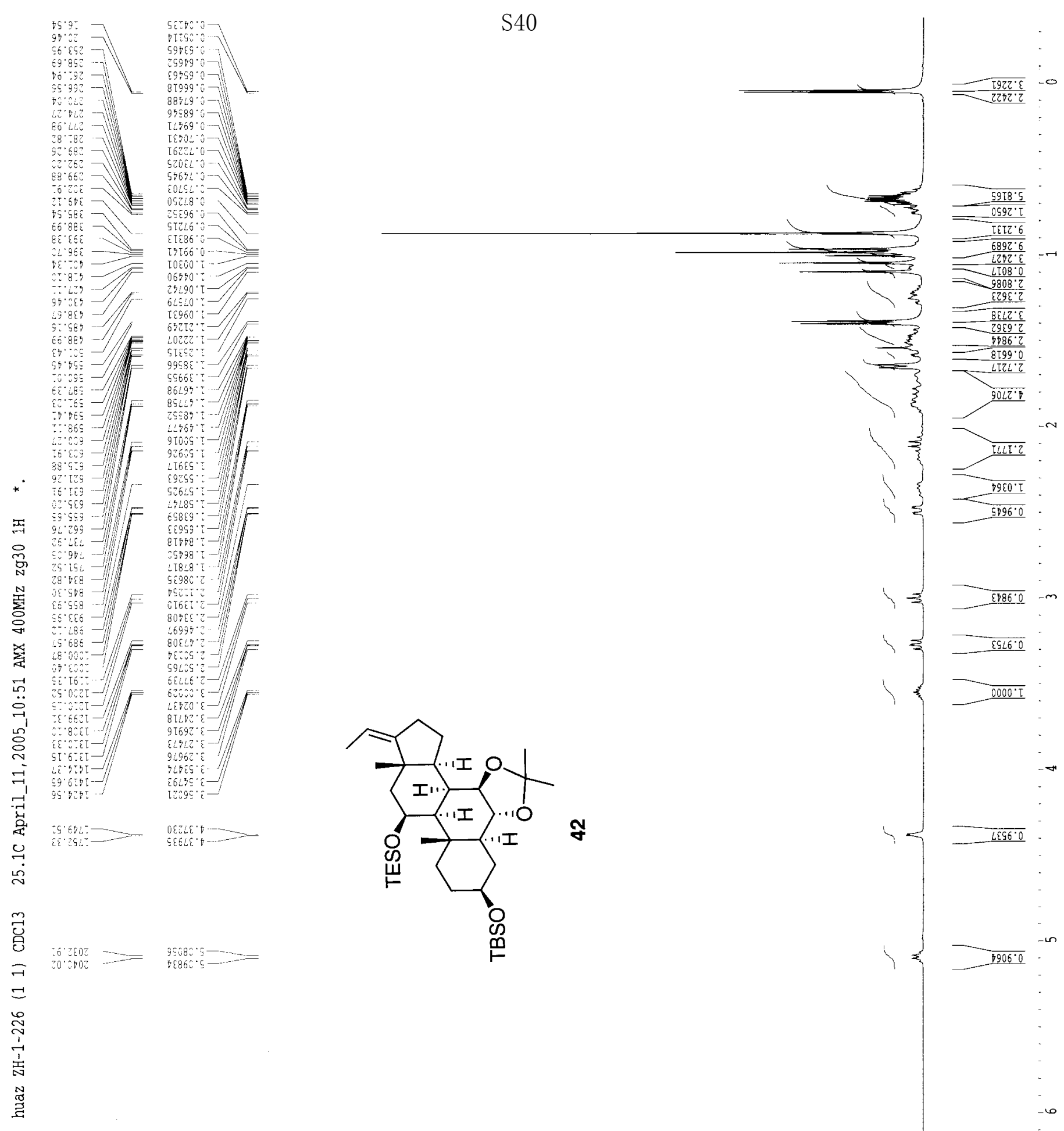

$20.5060-17092^{\circ}$ 


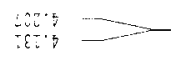

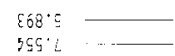

$855 \cdot \varepsilon T$

$8767: \square$

8098

$600.95-2$
750
600

$469 \cdot 2 i=$

$\angle 25 \cdot 22$

$599 \cdot 7$.

286.25

$26951-$

609.28

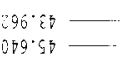

cag 2 ?
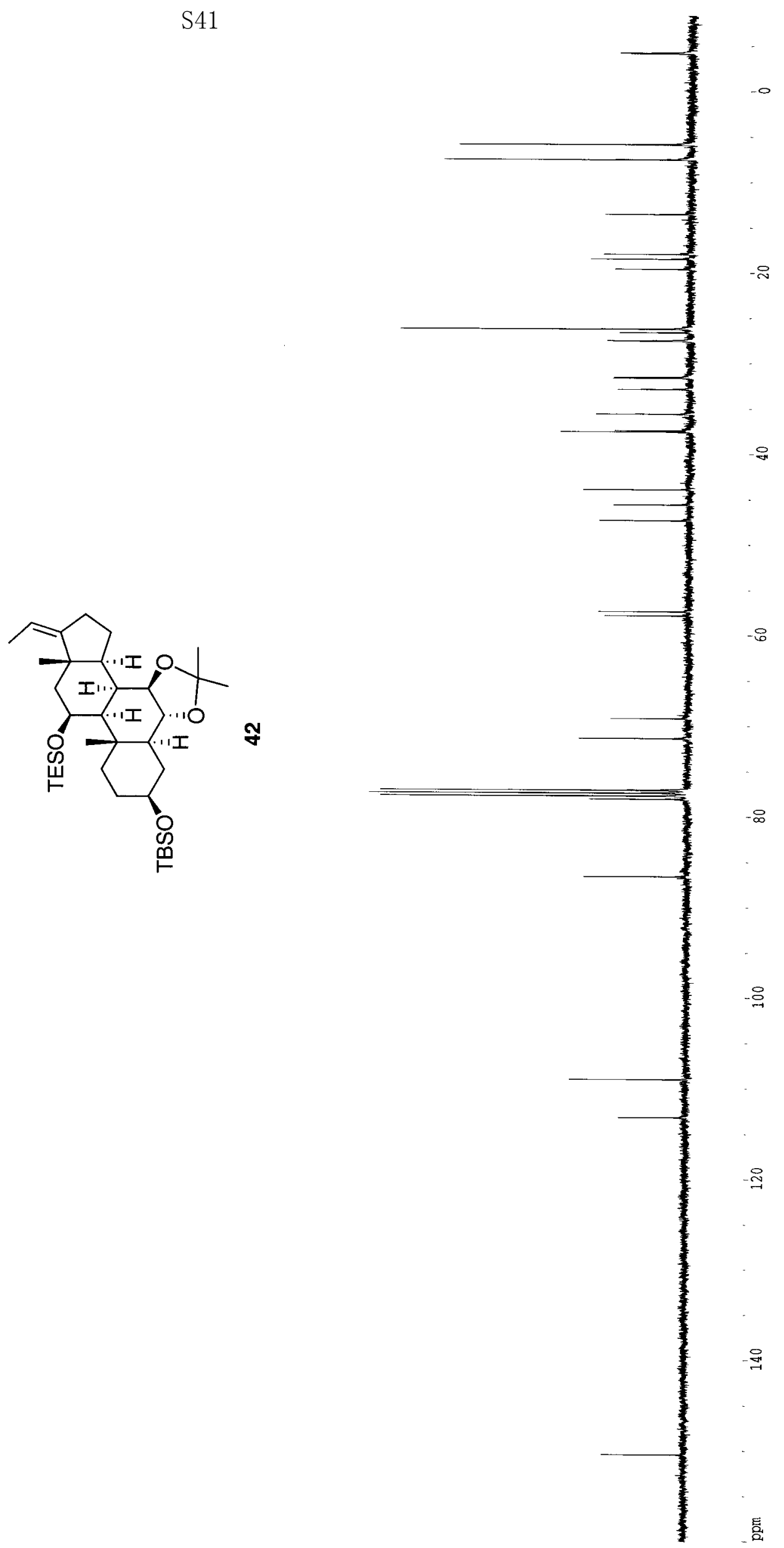

육 

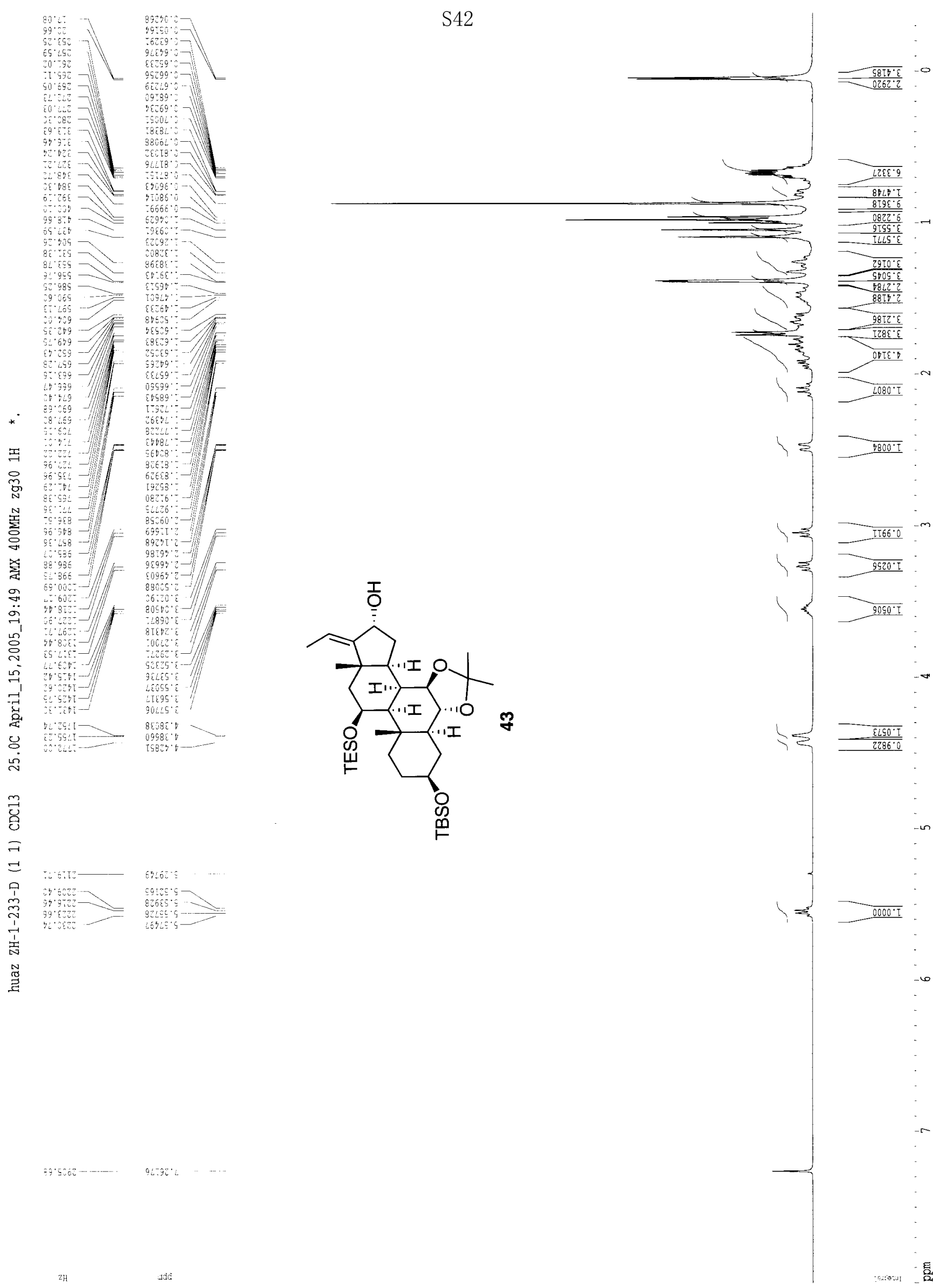

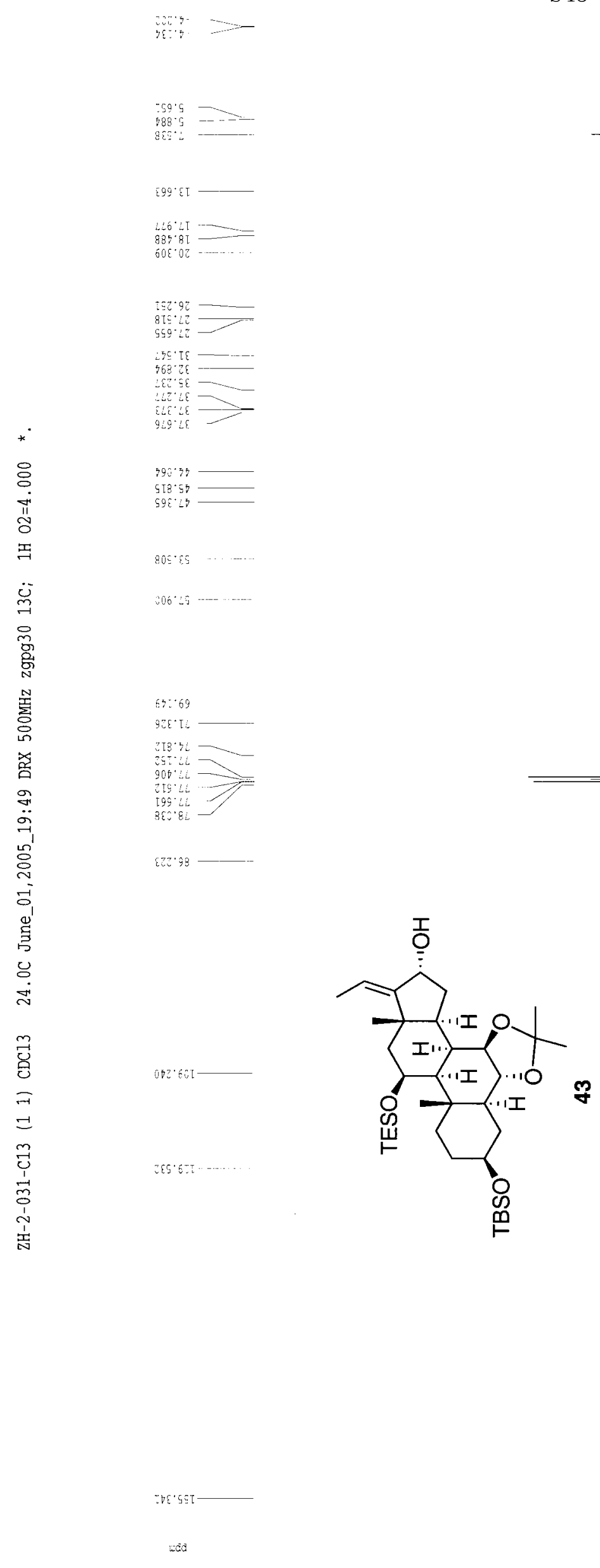


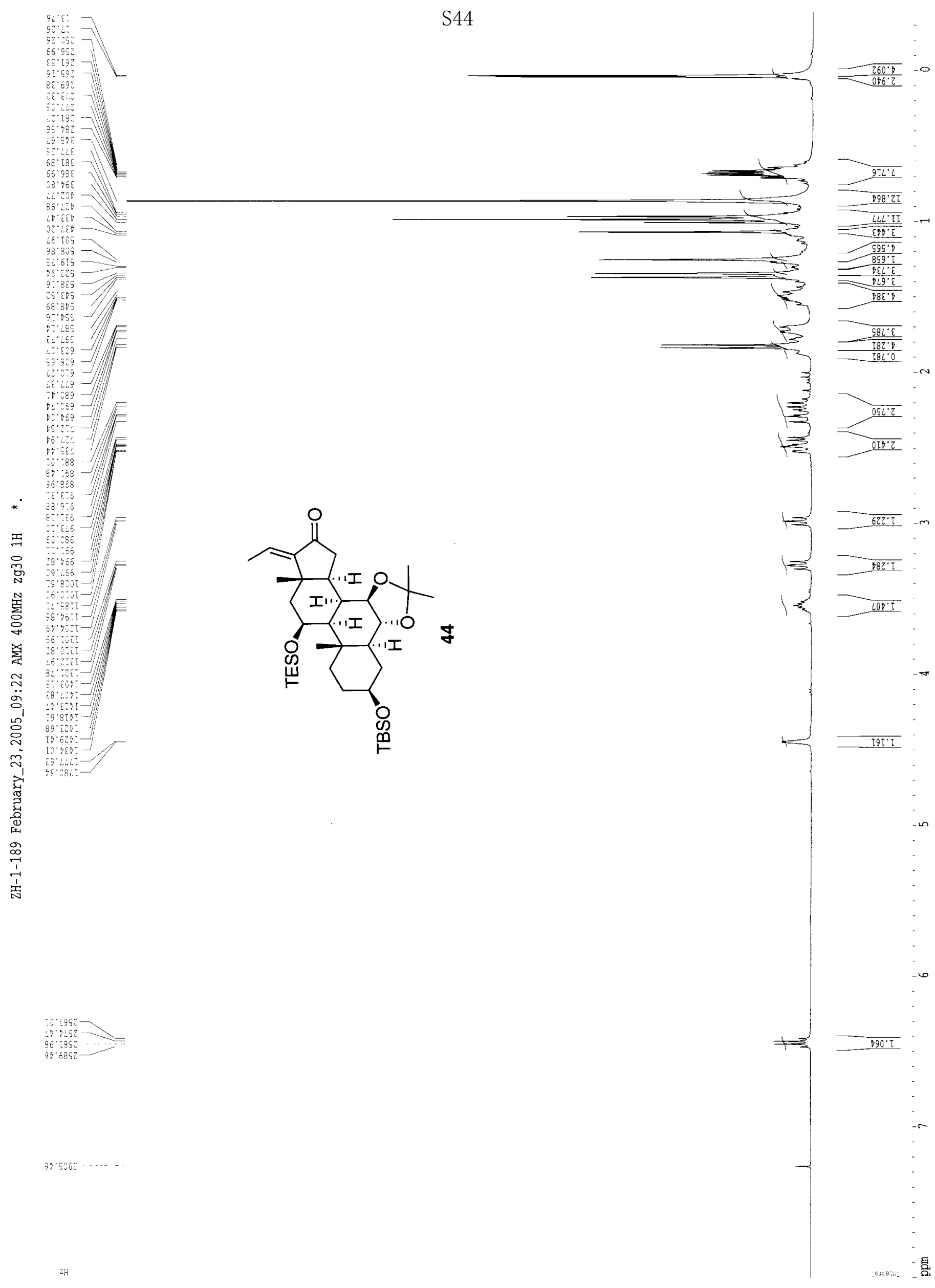




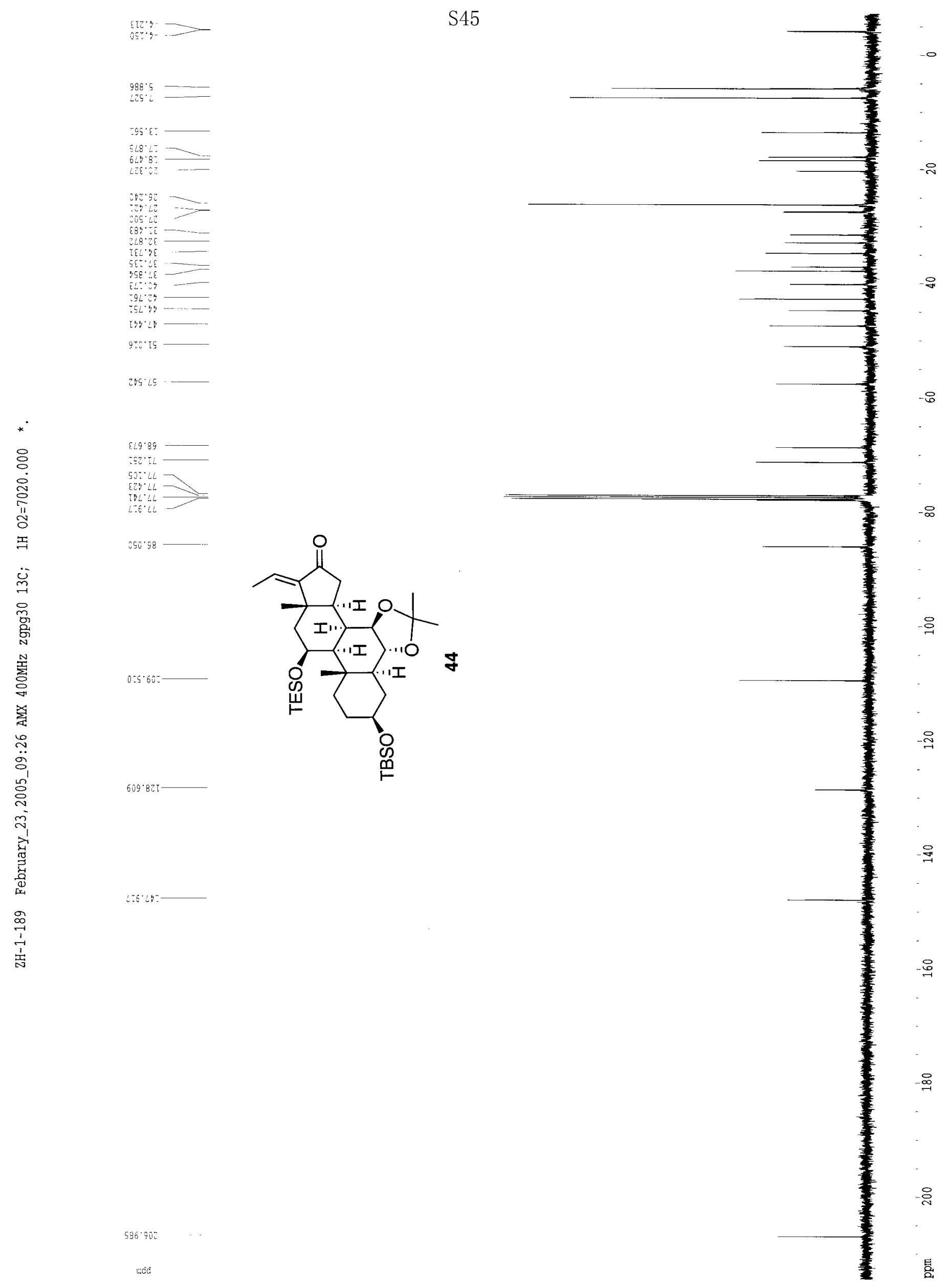




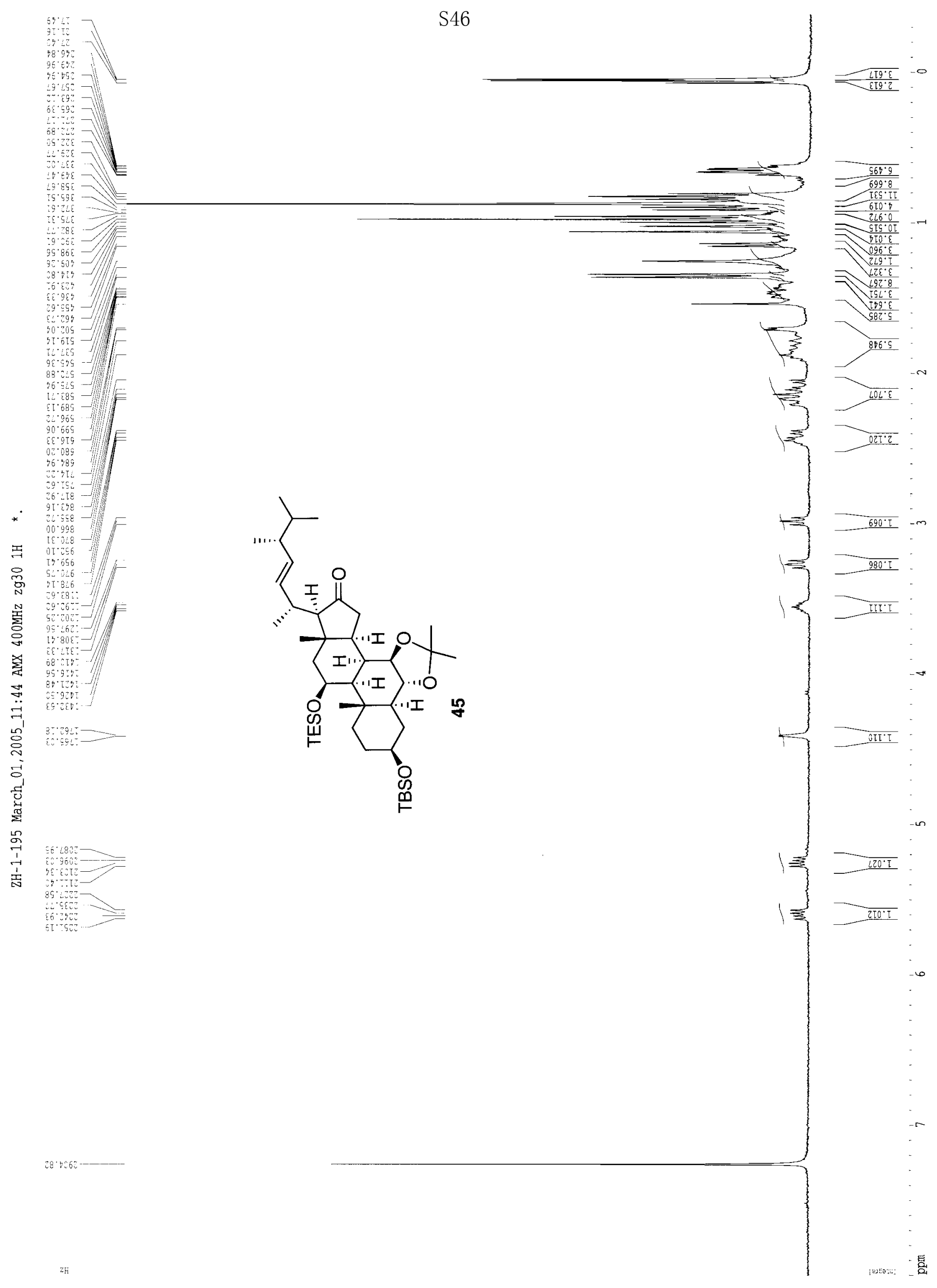




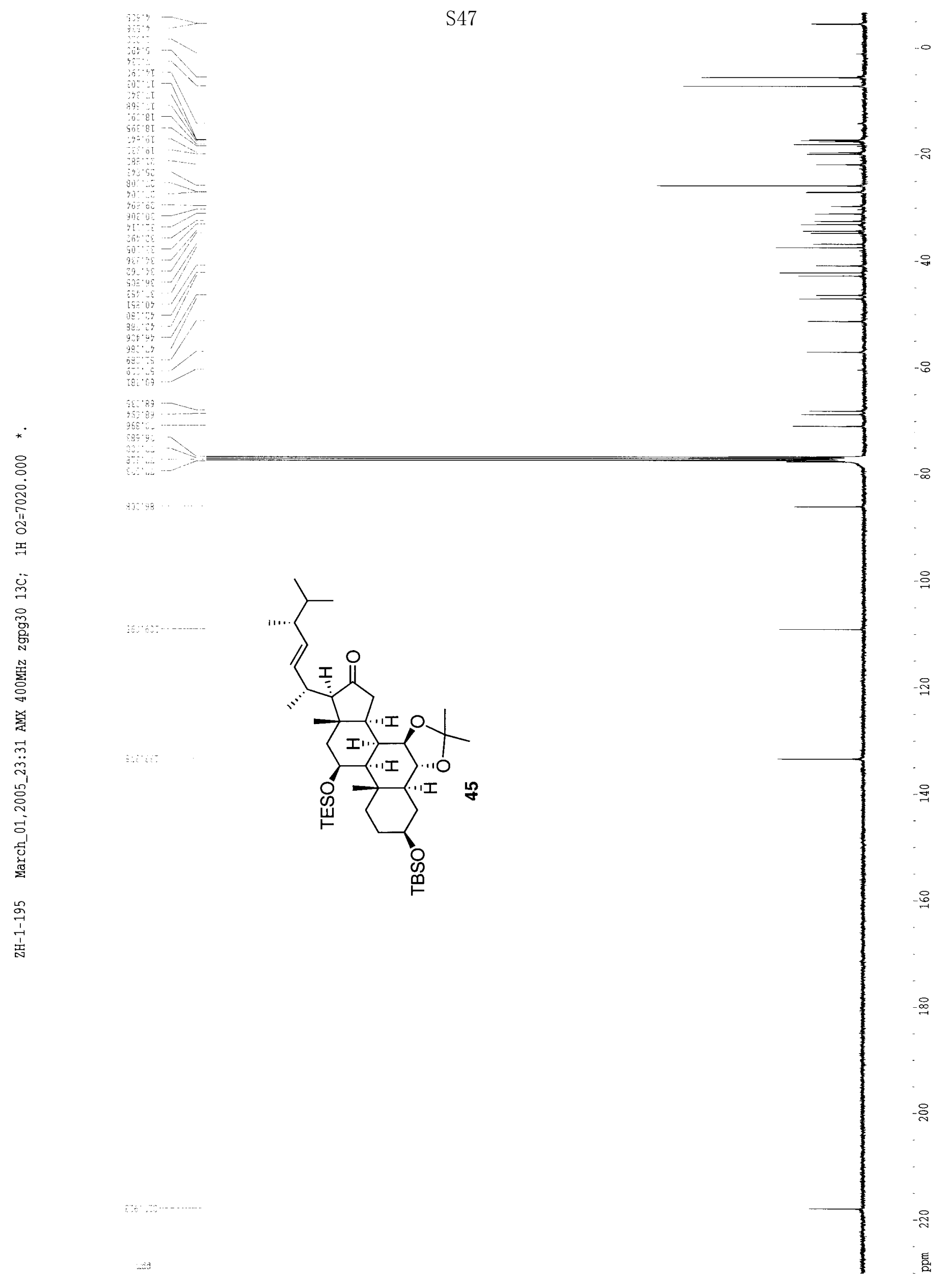




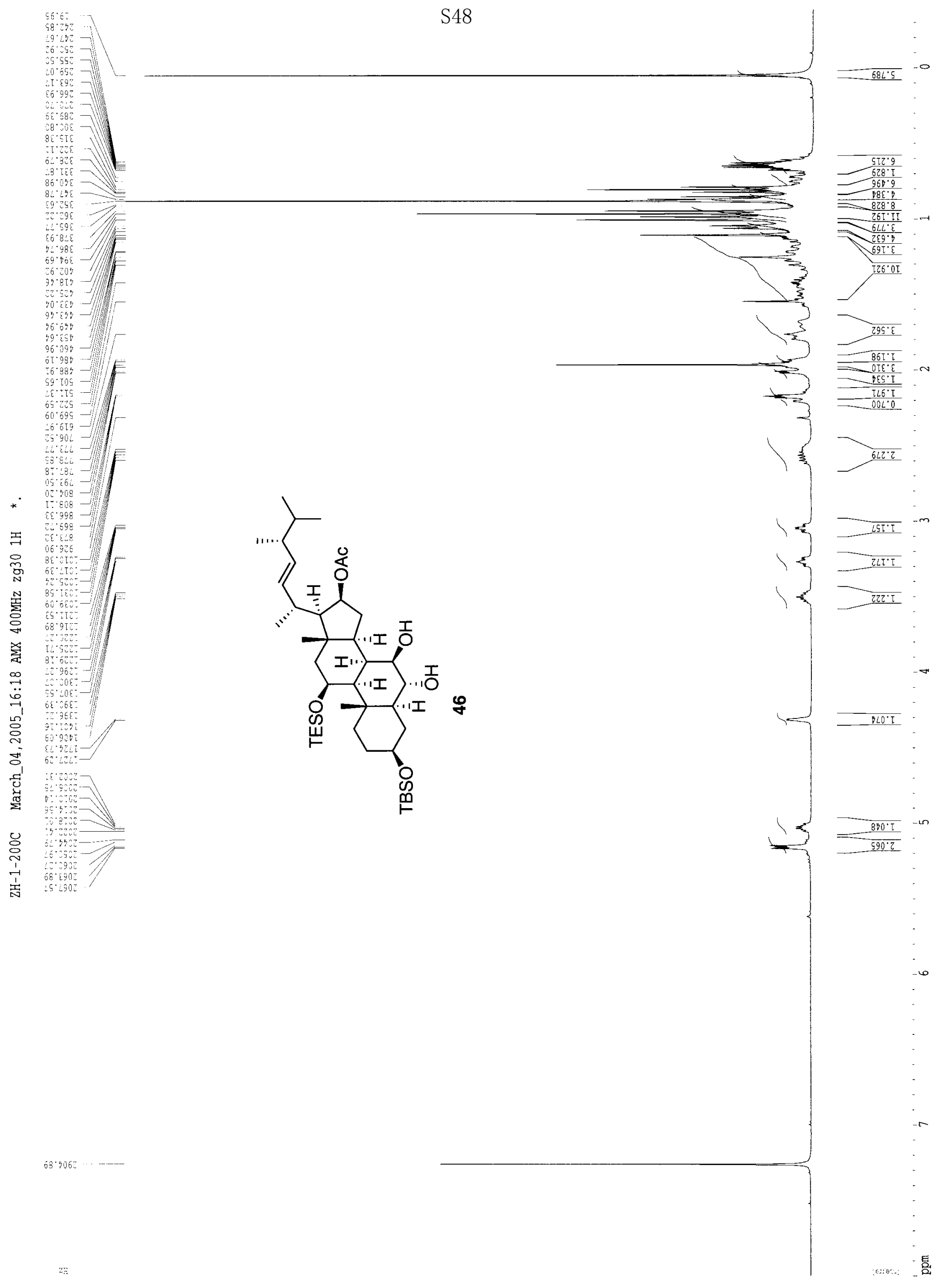



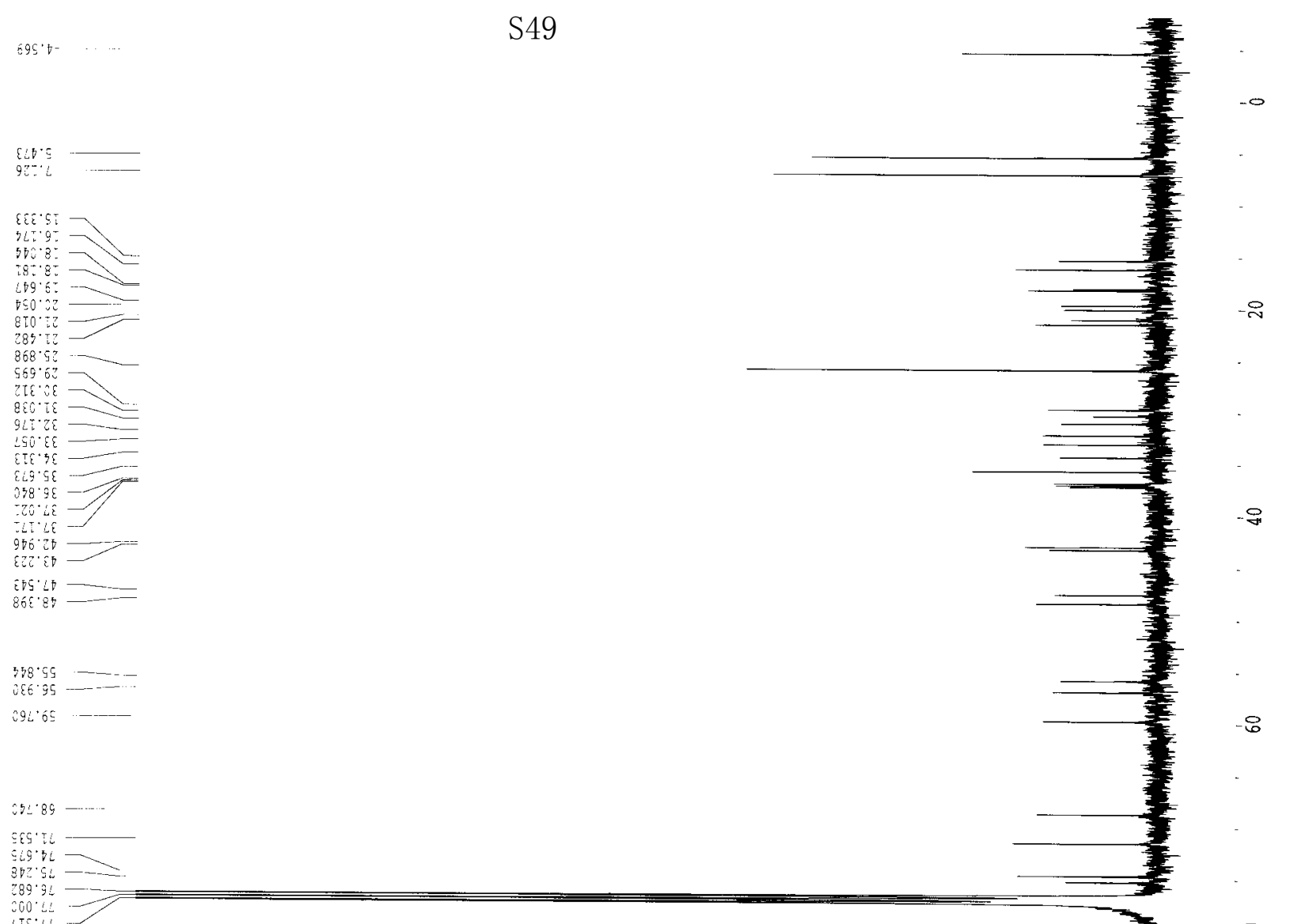

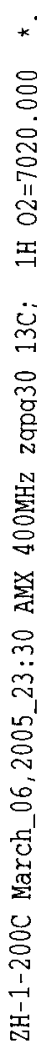

$358.59-1-.$.
$086.95-\ldots$

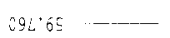

esc $T$.

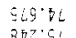

$88 \div 5=-1$

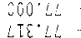

13800
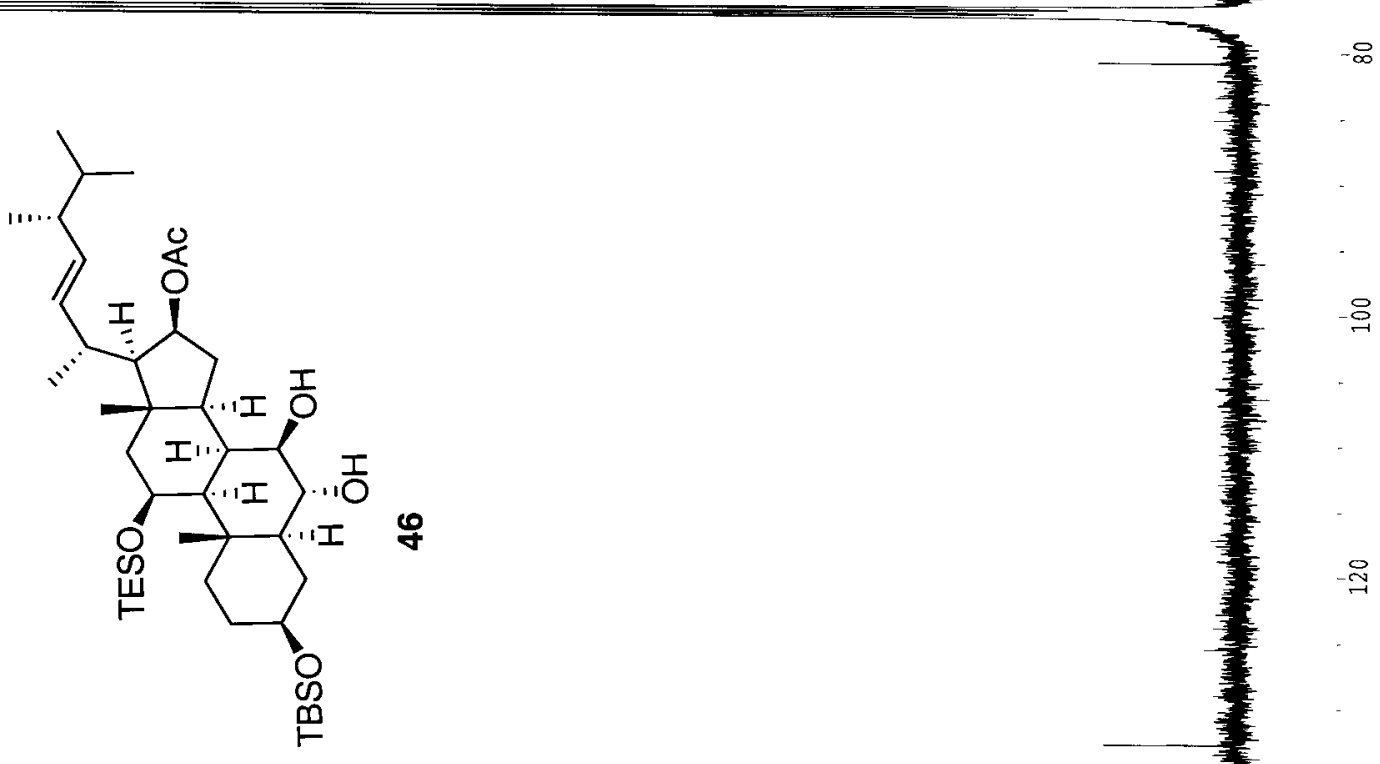

$68525:-\square-$
$29: 96:-\cdots-$

$\infty$

욤 
S50

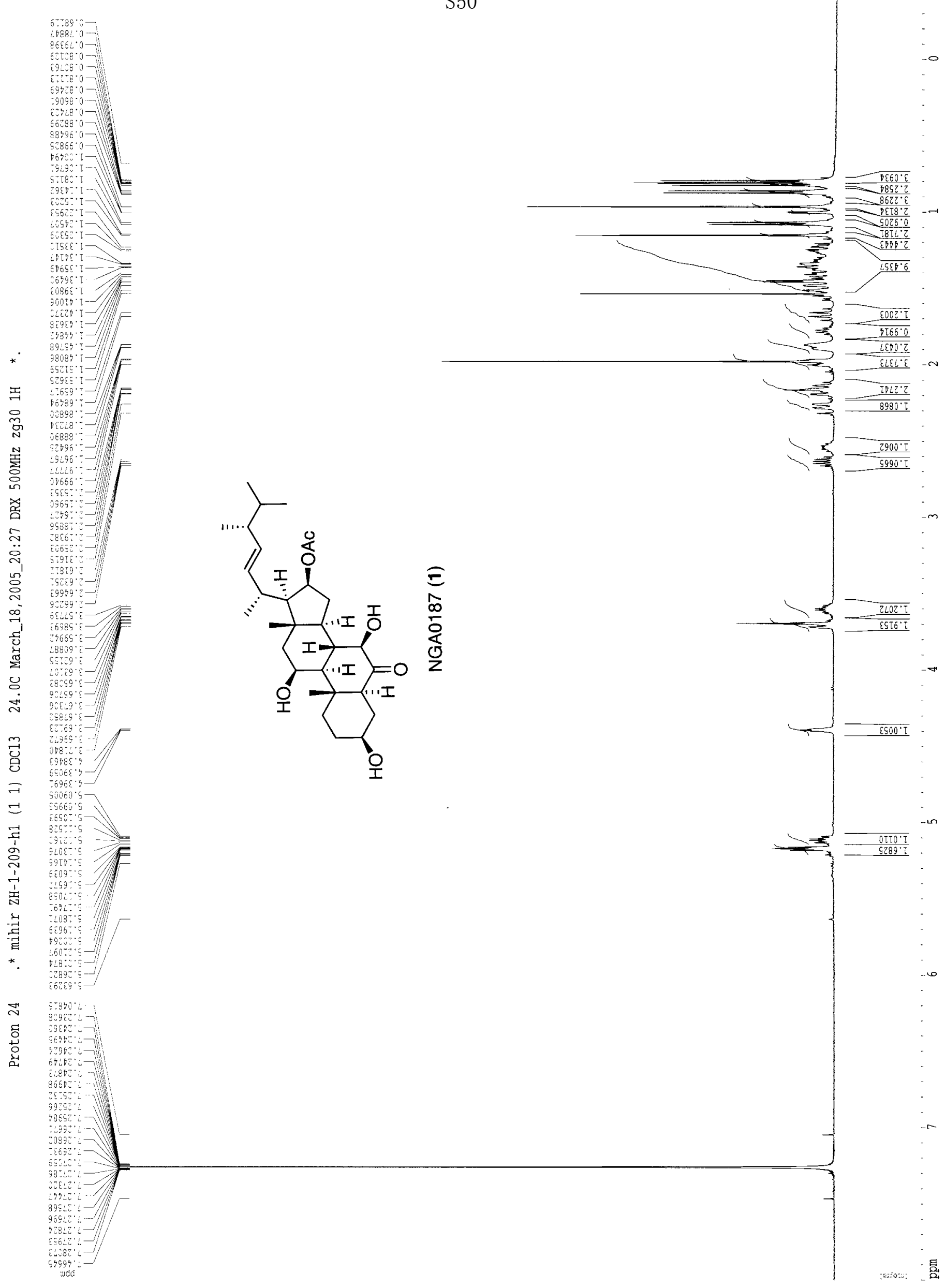




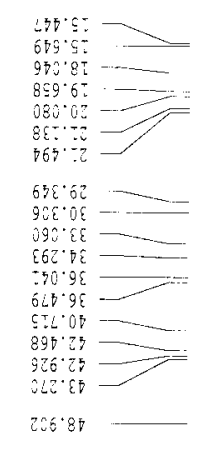

$589.05-$
$528.95-2$
$57.95-2$

$96: 09$

26839

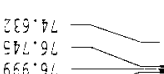

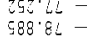

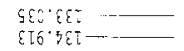

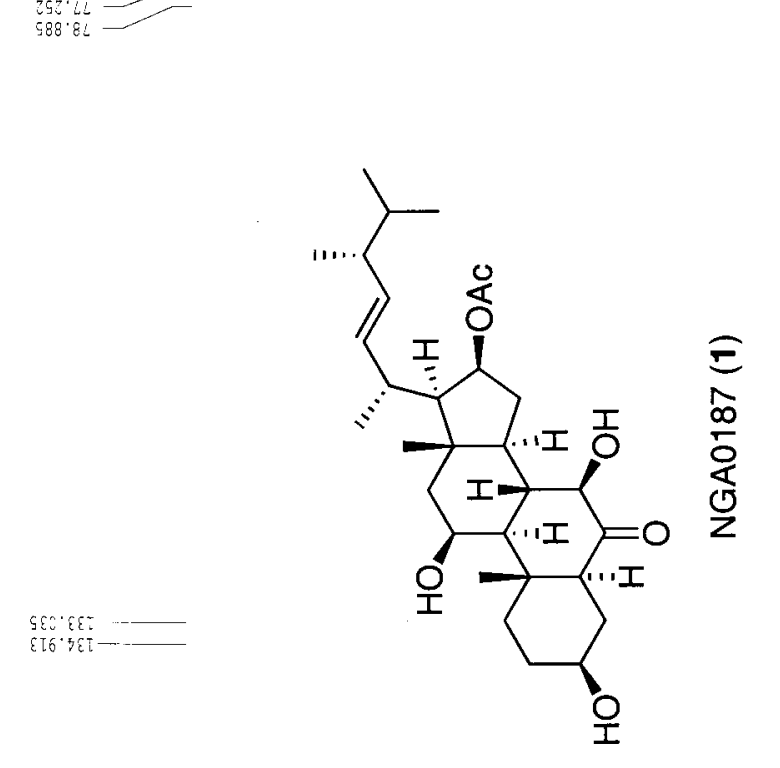

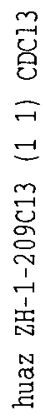

$\varepsilon 20.0 L T \ldots$

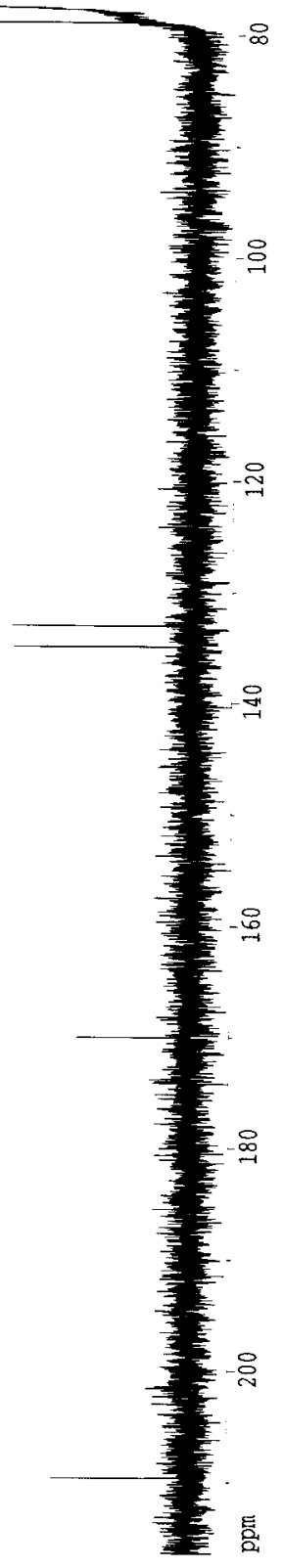

